\title{
NUMERICAL ANALYSIS OF AN ISOVOLUMETRIC THERMAL DESORPTION EXPERIMENT
}

\author{
A. von der Weth, K. Nagatou,
}

F. Arbeiter, R. Dagan, D. Klimenko, V. Pasler, M. Schulz, INR, KIT, CN, Athens, 25th June, 2019

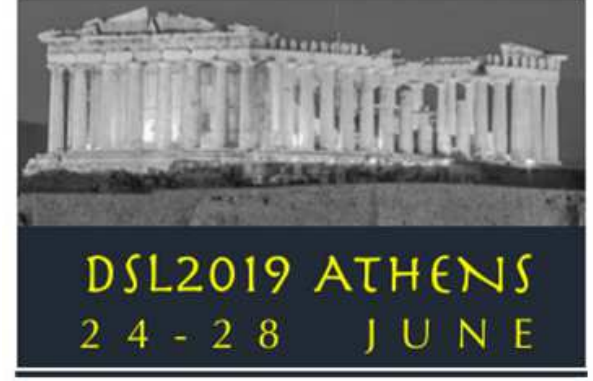

1.: Description of setup

2.: Simple analytical solutions

3.: Structure of Differential Equations

4.: Results of numerical optimization

5.: Outlook to analytical solution 


\section{Reminder: gas release diffusion experiments}

\section{Procedure:}

1. Specimen placed in defined volume enclusure

2. Load specimen with hydrogen at defined conditions $\left(T, \mathrm{p}_{\mathrm{H} 2}\right)$

3. Swiftly evacuate gases from enclosure

4. Measure pressure increase $p(t)$ in enclosure

$\rightarrow$ Sieverts' constant can be deduced from final pressure

$\rightarrow$ Diffusion constant can be deduced from pressure rise phase
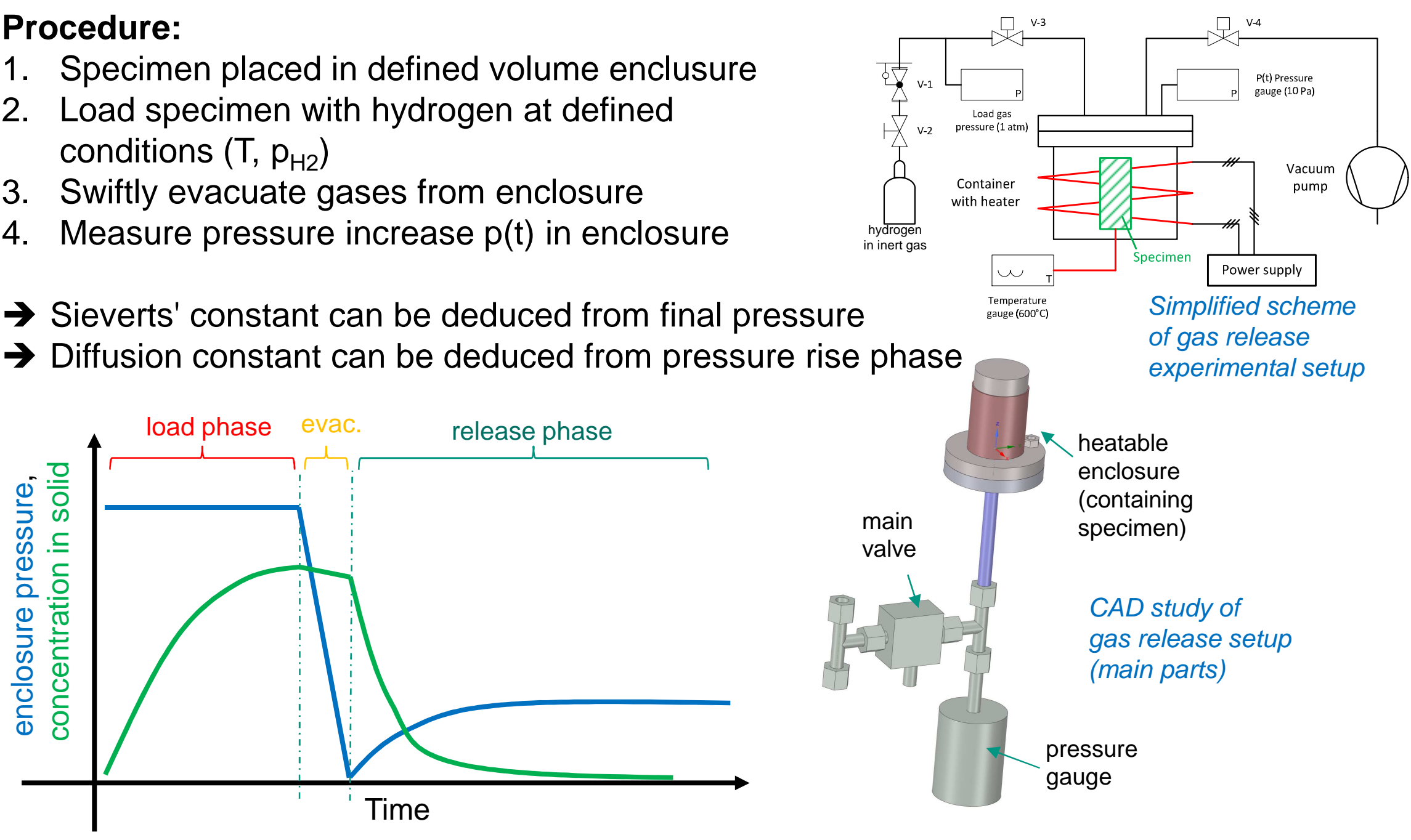


\section{1.: Description of setup of an Isovoluminetric Thermal}

Desorption Experiment

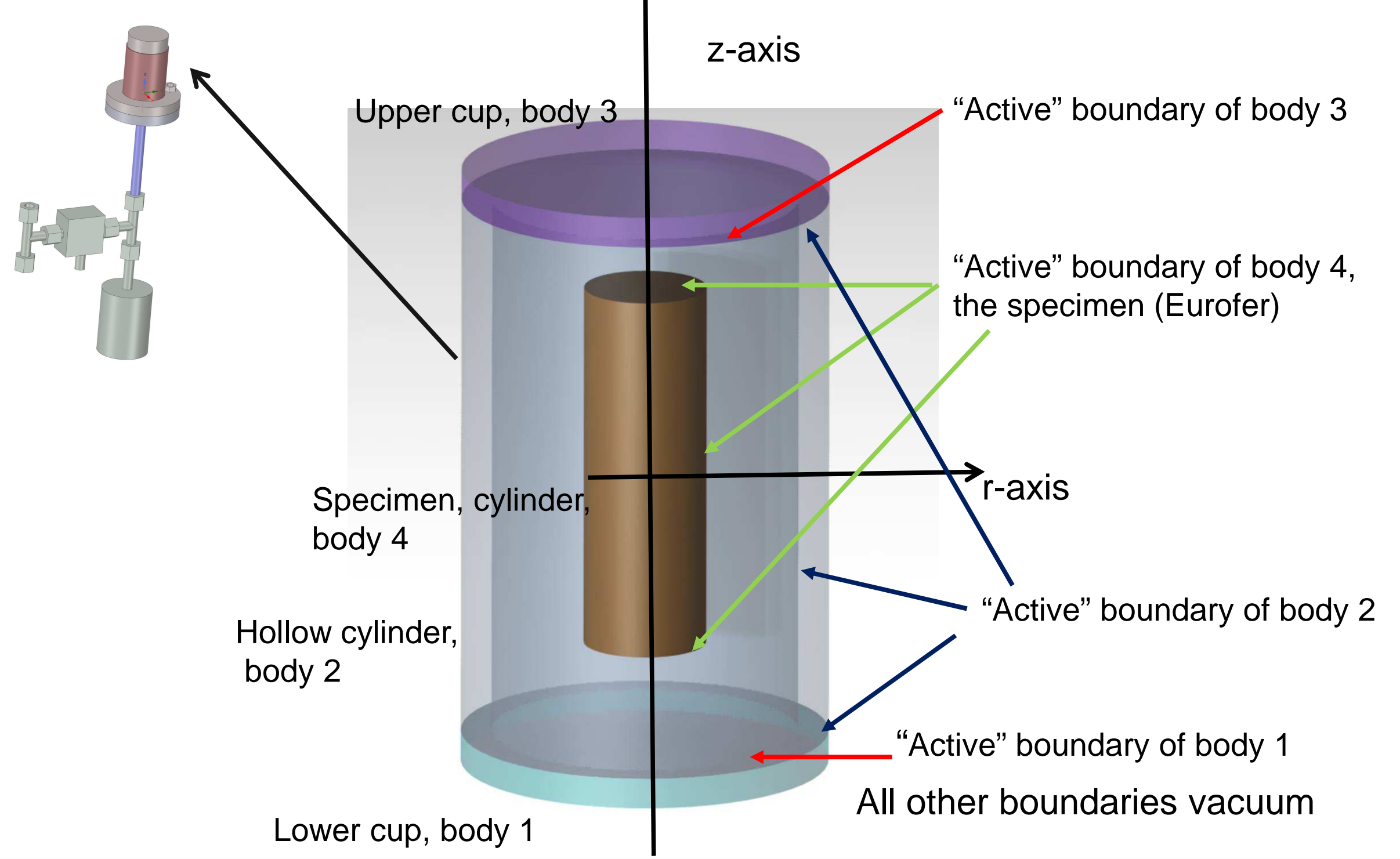




\section{1.: Physical description}

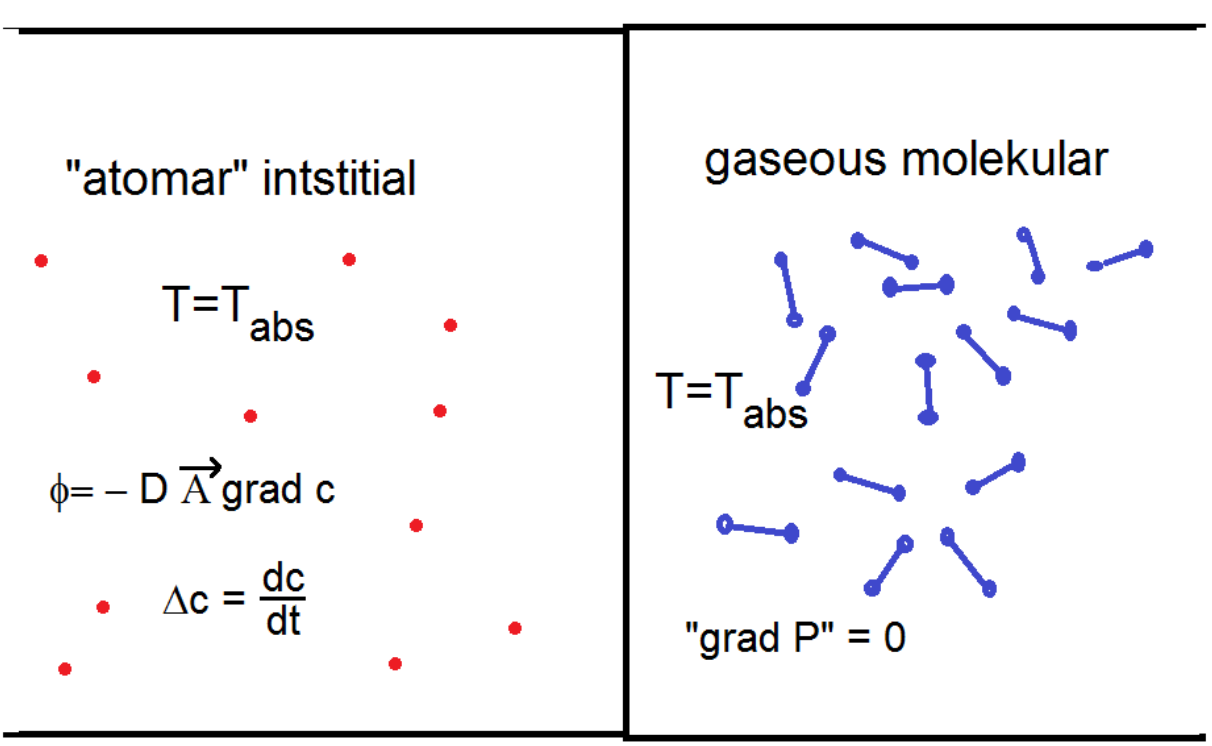

"Velocity" of mass transfer by diffusion constant D

Ratio of densities given by Sieverts' law (phase equilibrium)

$$
c=k_{s} \sqrt{p_{r}}
$$

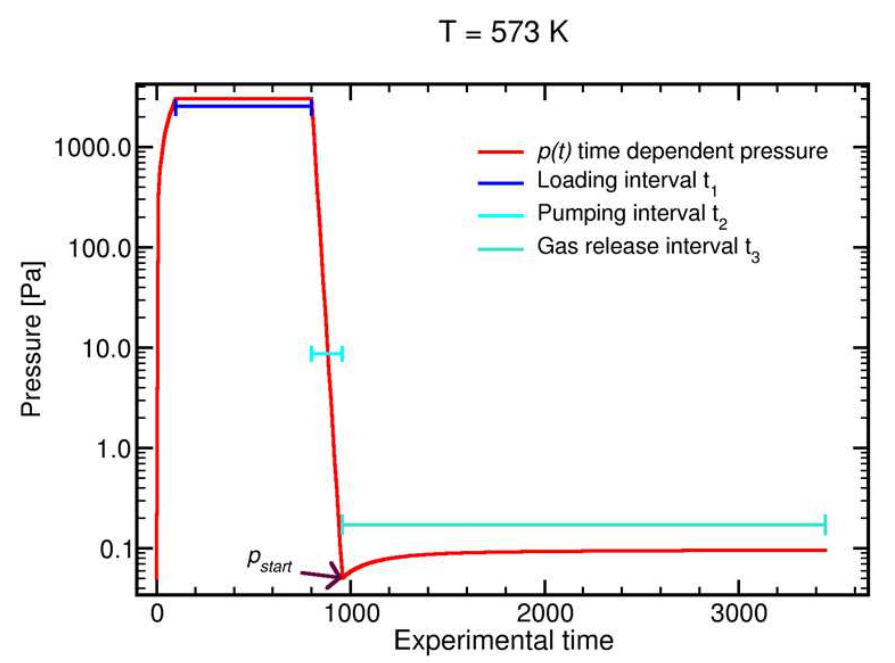

Removable cup

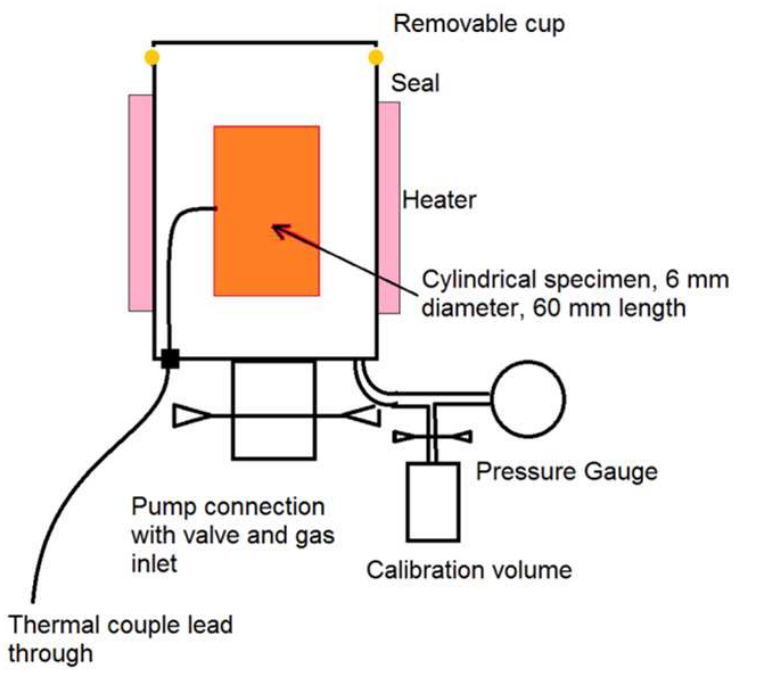




\section{2.: Simple analytical Solutions}

Without re-diffusion, complete outgassing, $c_{\text {sample }}=0$ :

$$
\underbrace{p_{r}}_{t \rightarrow \infty}=\underbrace{p_{\text {end }}}_{\begin{array}{c}
\text { switching off } \\
\text { pump }
\end{array}}+\frac{V_{s a} k_{s, s a} \sqrt{p_{\text {load }}} R T}{\begin{array}{c}
V_{c}-V_{\text {chamer }}-V_{\text {sample }} \\
\text { sample }
\end{array}}
$$

With phase equilibrium, mass conservation (number of "hydrogens" in atomic interstitial and molecular gaseous state constant) and non interacting confinement condition, currently unused:

$$
0=\frac{2\left(V_{c}-V_{s a}\right)}{R T}\left(\sqrt{p_{r}}\right)^{2}+V_{s} k_{s, s a} \underbrace{\sqrt{p_{r}}}_{=" x^{\prime \prime}}-\left(V_{s} k_{s, s a} \sqrt{p_{\text {load }}}+\frac{2 p_{\text {end }}\left(V_{c}-V_{s a}\right)}{R T}\right)
$$

$$
k_{s, s a}=\frac{2\left(V_{c}-V_{s a}\right)}{R T V_{s a}} \frac{\left(p_{r}-p_{\text {end }}\right)}{\sqrt{p_{\text {load }}-\sqrt{p_{r}}}}
$$

Experimentally difficult realization of boundaries, no statement about diffusion constant

$$
\underbrace{p_{r}}_{t \rightarrow \infty}=\left(\frac{-1 \underbrace{+}_{n e g . s q r t} \sqrt{1+\left(\frac{8\left(V_{c}-V_{s a}\right) \sqrt{p_{\text {load }}}}{R T k_{s, s a}}+\frac{16 p_{\text {end }}\left(V_{c}-V_{s a}\right)^{2}}{\left(R T V_{s a} k_{s, s a}\right)^{2}}\right)}}{\left(\frac{V_{s a} k_{s, s a} R T}{4\left(V_{c}-V_{s a}\right)}\right)^{-1}}\right)^{2}
$$




\section{3.: Structure of differential equations:}

$$
\begin{aligned}
& \frac{\partial c}{\partial t}=D_{s a} \Delta c \quad \frac{\partial d(i)}{\partial t}=D_{c u} \Delta d(i), i=1,2,3 \\
& \Delta=\frac{\partial^{2}}{\partial r^{2}}+\frac{1}{r} \frac{\partial}{\partial r}+\frac{\partial^{2}}{\partial z^{2}}+\underbrace{\frac{1}{r^{2}} \frac{\partial^{2}}{\partial \varphi^{2}}}_{=0} \\
& c\left(0 \leq r \leq r_{s}, z= \pm z_{s}, \forall t\right)=k_{s, s a} \sqrt{p(t)} \\
& c\left(r=r_{s},|z| \leq z_{s}, \forall t\right)=k_{s, s a} \sqrt{p(t)} \\
& d(1)\left(r \leq r_{c o}, z=-z_{c i}, \forall t\right)=k_{s, c u} \sqrt{p(t)} \\
& d(2)\left(r=r_{c i},-z_{c i} \leq z \leq z_{c i}, \forall t\right)=k_{s, c u} \sqrt{p(t)} \\
& d(2)\left(r_{c i} \leq r \leq r_{c o}, z= \pm z_{c i}, \forall t\right)=k_{s, c u} \sqrt{p(t)} \\
& d(3)\left(r \leq r_{c o}, z=z_{c i}, \forall t\right)=k_{s, c u} \sqrt{p(t)}
\end{aligned}
$$

Analytical 1d: Sedano, Perujo (1999), Esteban Douglas (2001) , Eichenauer Pebler (1957) Hattenbach (1961)

Analytical 2d: Eichenauer, Pebler, Witte 1965

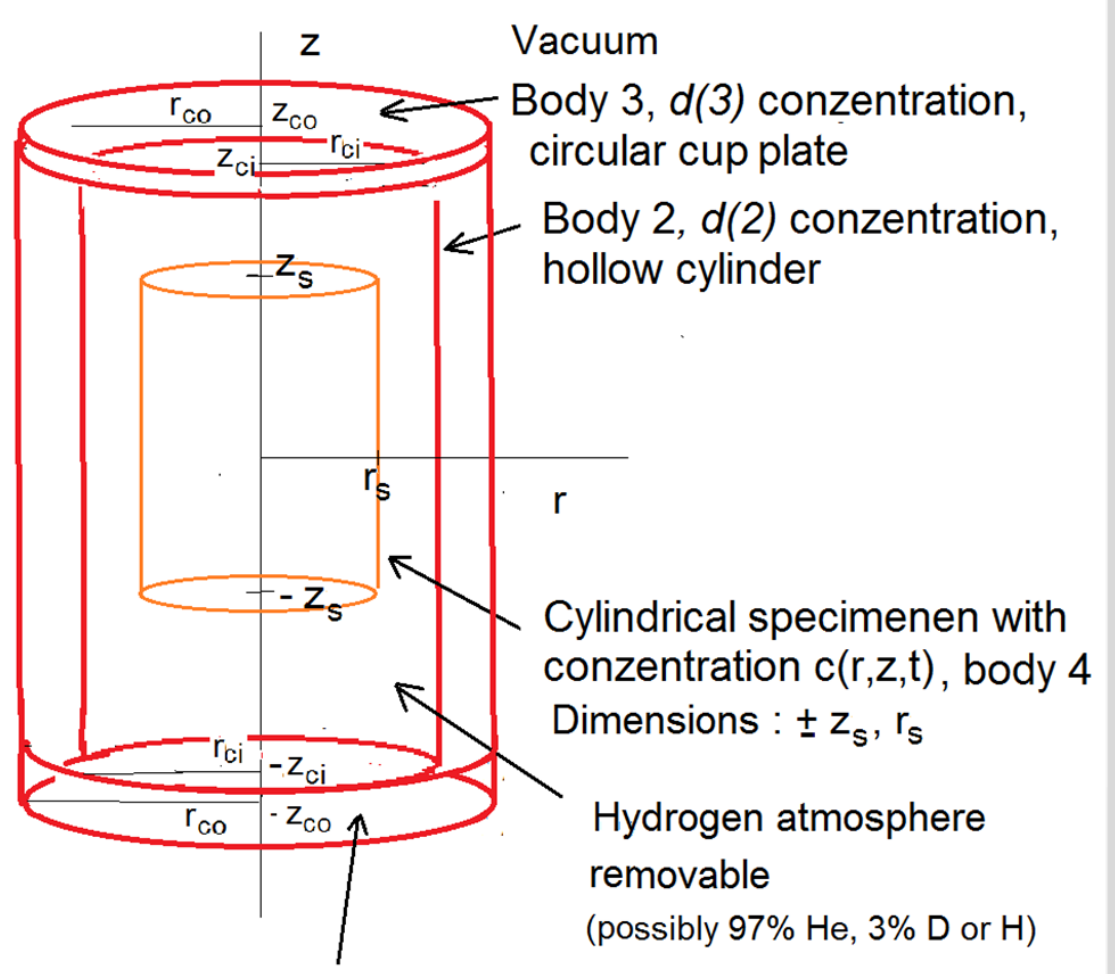

Body 1, $d(1)$ conzentration 


\section{Structure of differential equations}

$$
\frac{d m}{d t}=-2 \pi D_{s a} \int_{0}^{r_{s}} \underbrace{2}_{\text {symmetric }} r \frac{\partial}{\partial z} c\left(r, z=z_{s}, t\right) d r
$$

- $\underbrace{2 \pi r_{s} D_{s a} \int_{-z_{s}}^{z_{s}} \frac{\partial}{\partial r} c\left(r=r_{s}, z, t\right) d z}_{\text {superficies surface }}$

superficies surface of specimen

- $\underbrace{2 \pi D_{c u} \int_{0}^{r_{c o}} r \frac{\partial}{\partial z} d(1)\left(r, z=-z_{c i}, t\right) d r}$ circular aerea of body 1

- $2 \pi r_{c i} D_{c u} \int_{-z_{c i}}^{z_{c i}} \frac{\partial}{\partial r} d(2)\left(r=r_{c i}, z, t\right) d z$ superficies surface of body 2 $-\underbrace{2 \pi D_{c u} \int_{0}^{r_{c o}} r \frac{\partial}{\partial z} d(3)\left(r, z=z_{c i}, t\right) d r}_{\text {circular area of body } 3}$

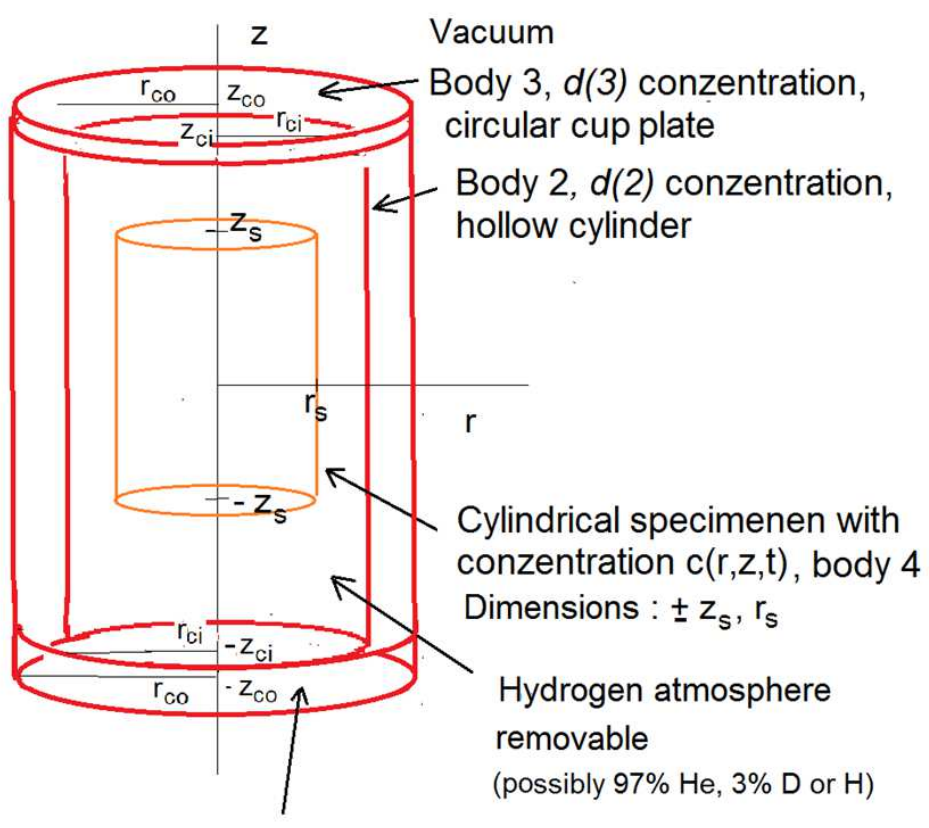

Body 1, d(1) conzentration

$$
p(t)=p_{\text {start }}+\underbrace{k_{v}}_{R T_{\text {abs }} / V_{\text {gas }}} \int_{t_{1}+t_{2}}^{t} \underbrace{0.5}_{\begin{array}{c}
\text { gaseous } \\
\text { interstitial }
\end{array}} \frac{d m}{d t} d t
$$




\section{4.: Results of numerical solution}

$\mathrm{T}=773 \mathrm{~K}, \mathrm{Cu}$ containment with Optifer specimen, 13. 5. chamber dimension $80 \mathrm{~mm}$ to $40 \mathrm{~mm}$ diameter, $\mathrm{p}_{\text {load }}=310^{3} \mathrm{~Pa}$, improved gradient calculation

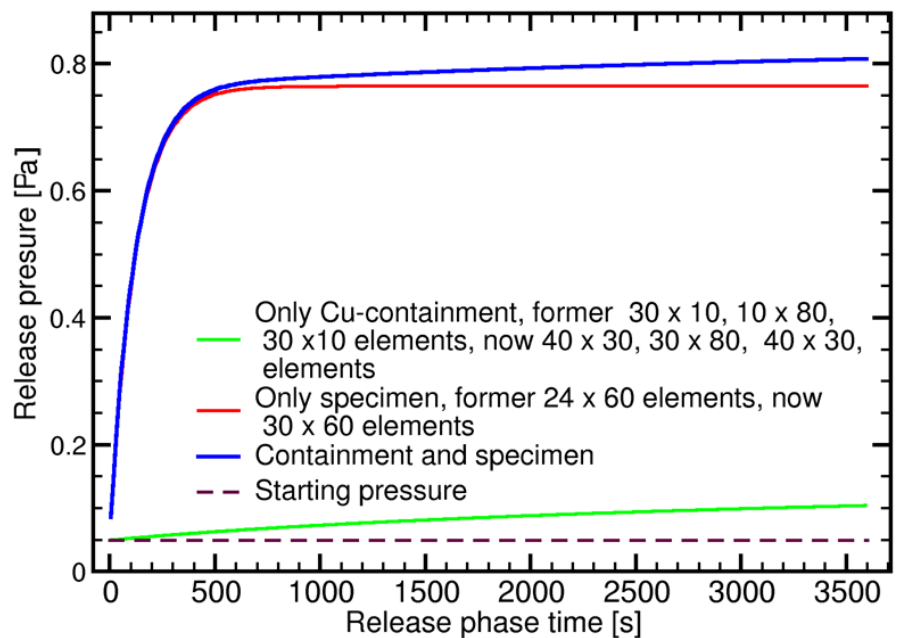

Simple numerical gradient calculation

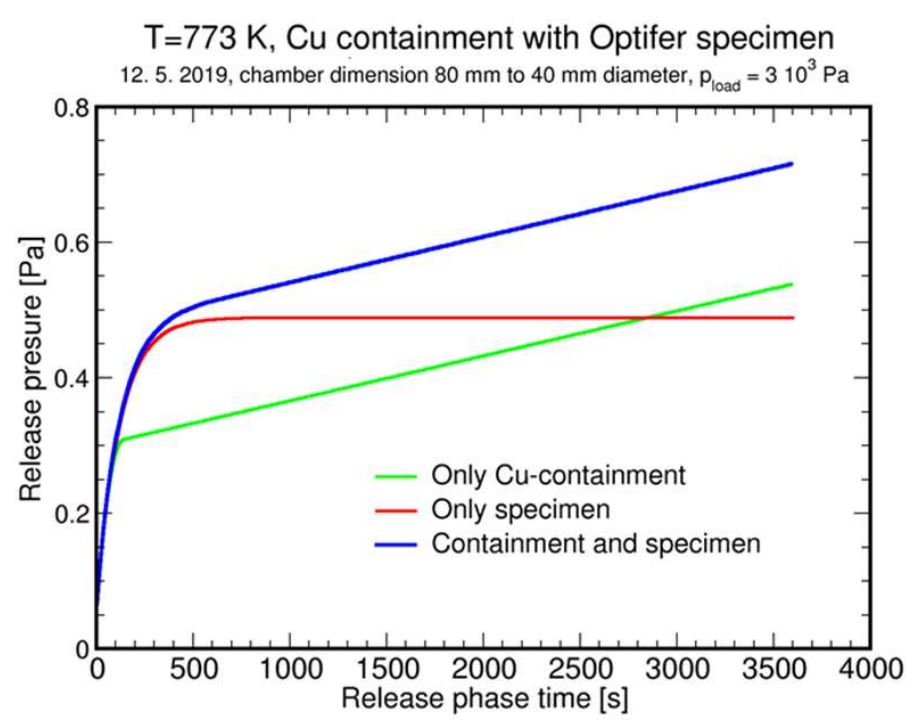

\section{Numerical artefacts}

Development, $\mathrm{T}=773 \mathrm{~K}$, Cu cont. with Optifer specimen chamber dimension $80 \mathrm{~mm}$ to $40 \mathrm{~mm}$ diameter, $\mathrm{p}_{\text {load }}=310^{3} \mathrm{~Pa}$, improved gradient calculation

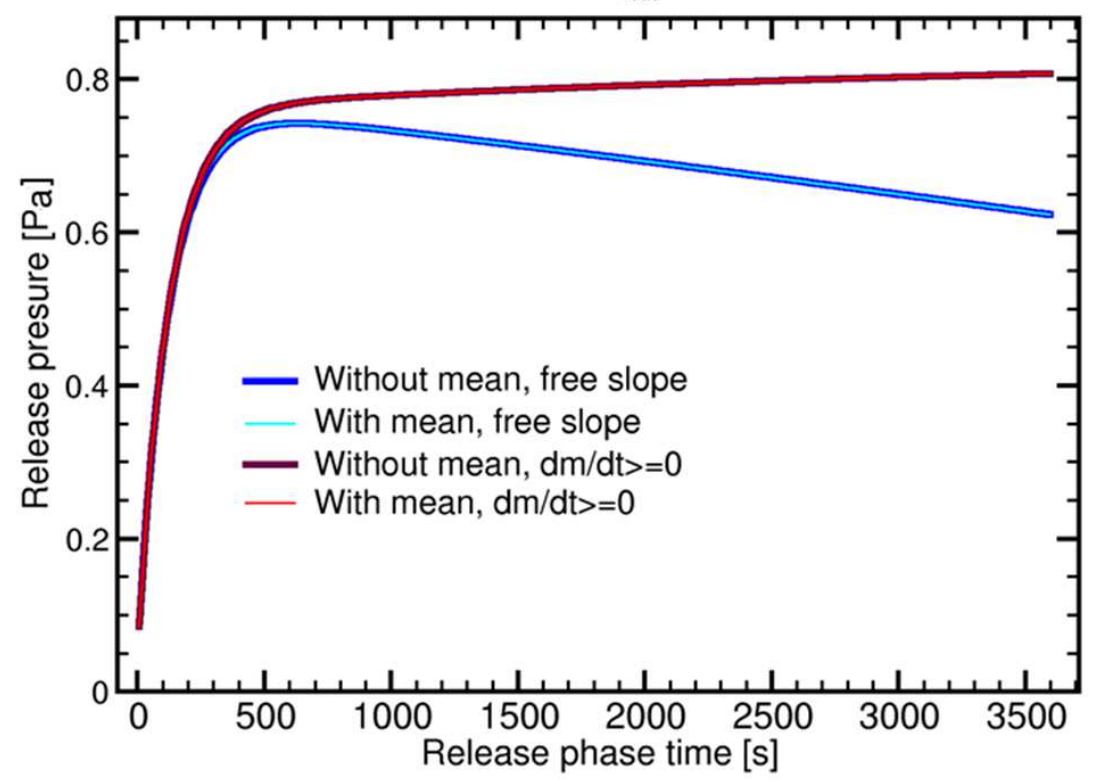




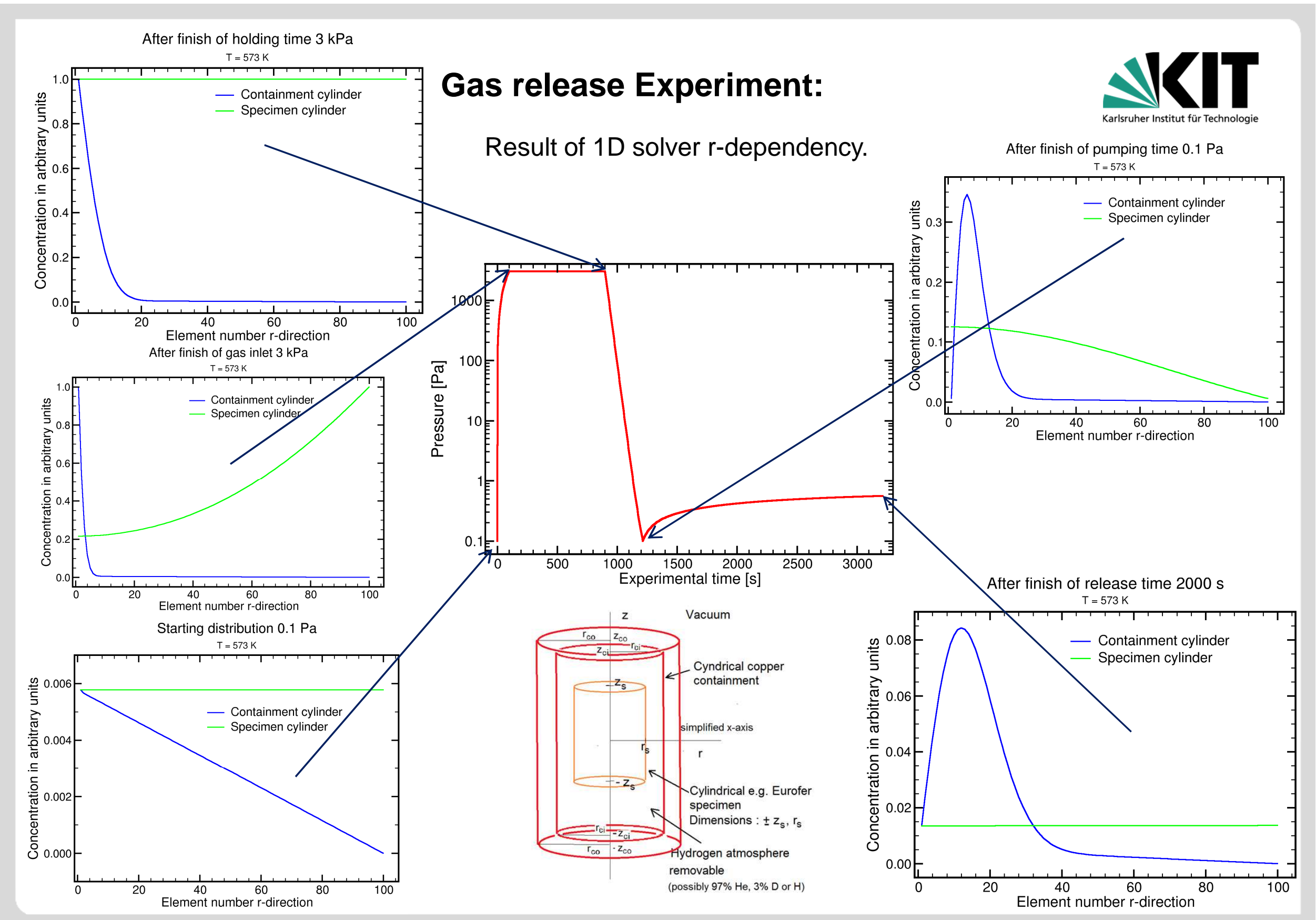

$925^{\text {th }}$ of June, NUMERICAL ANALYSIS OF AN ISOVOLUMETRIC THERMAL DESORPTION EXPERIMENT

MET/INR/Maschinenbau 


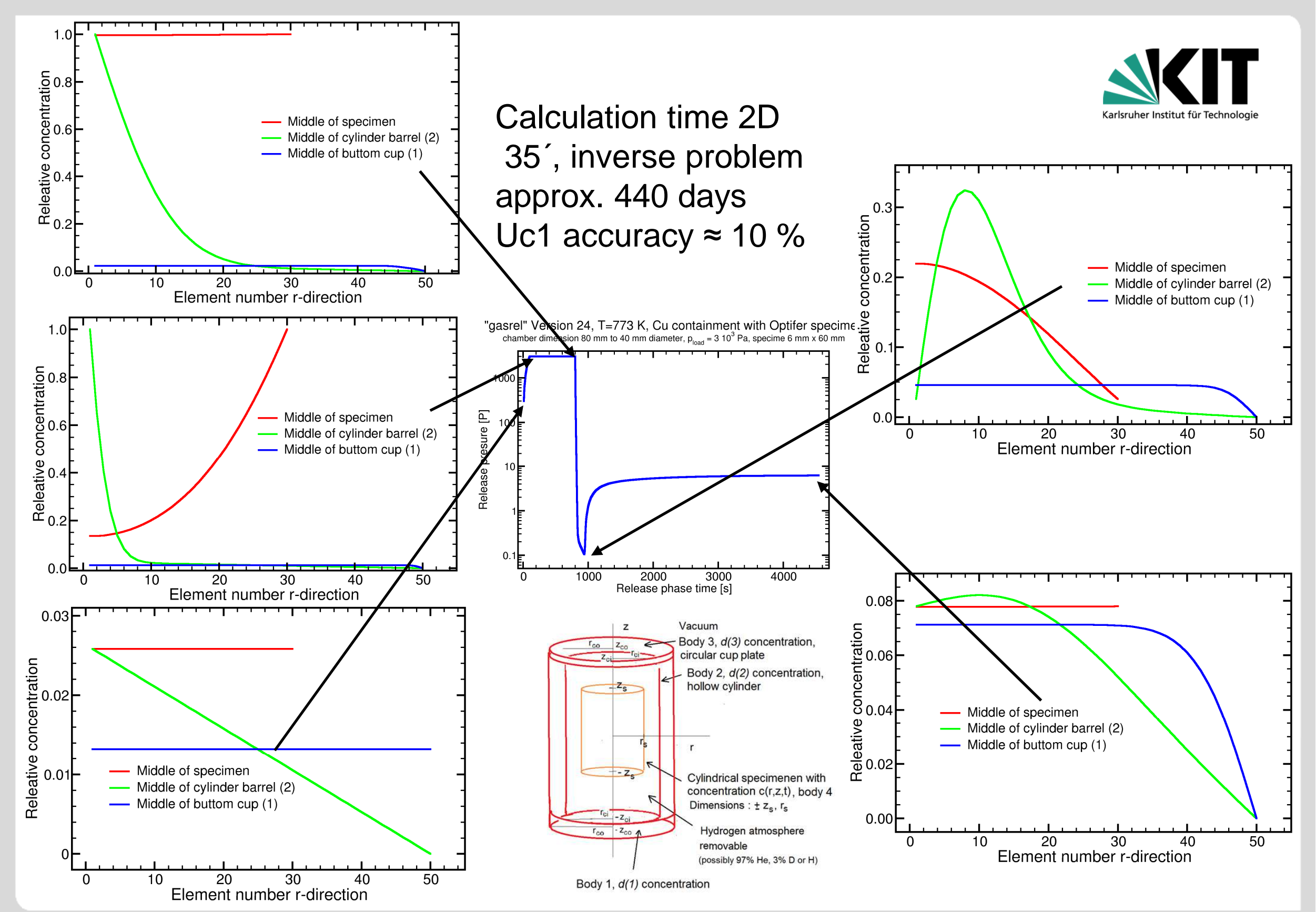




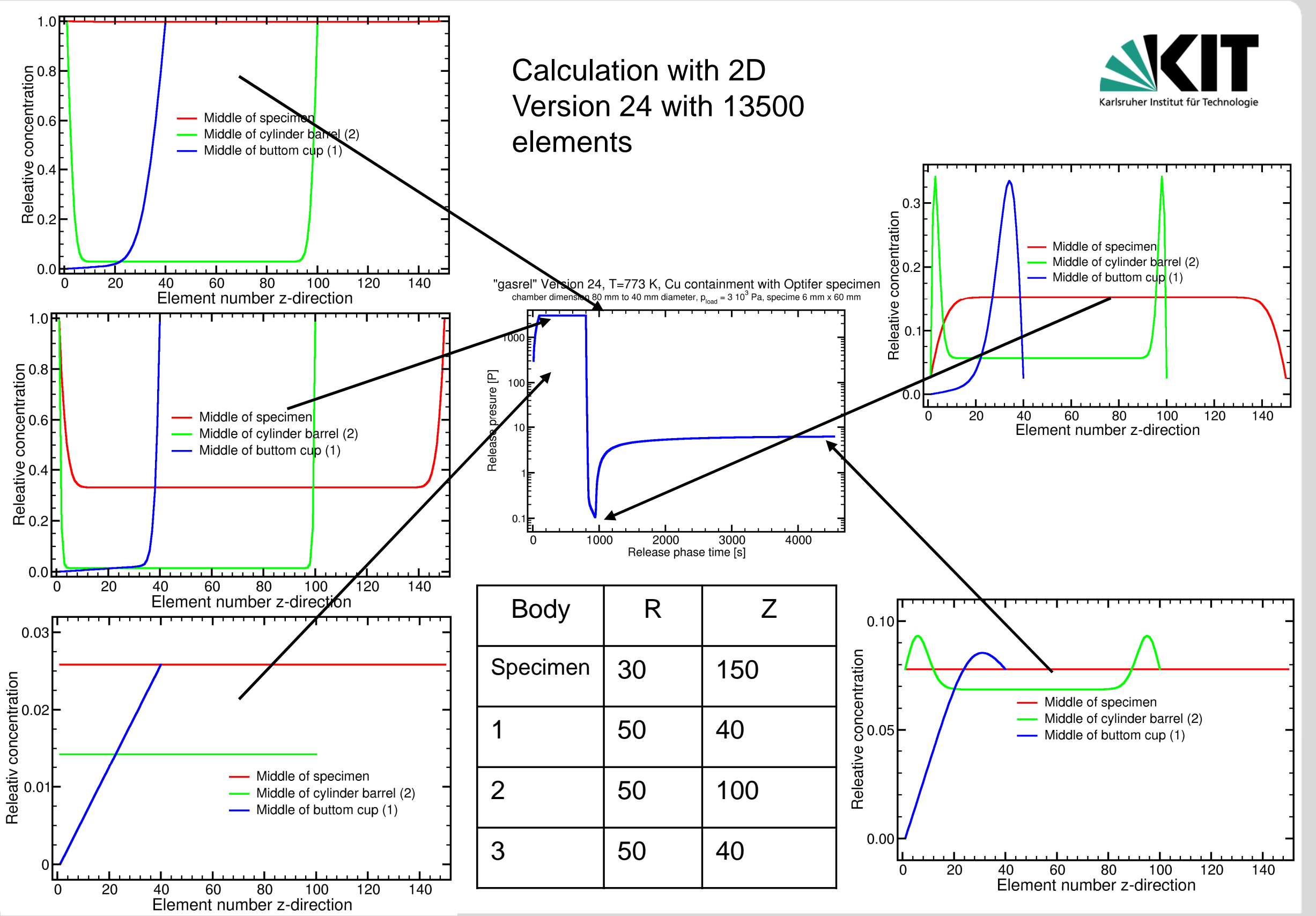

$1125^{\text {th }}$ of June, NUMERICAL ANALYSIS OF AN ISOVOLUMETRIC THERMAL DESORPTION EXPERIMENT

MET/INR/Maschinenbau 


\section{Comparison with analytical solution:}

Results for chamber $40 \mathrm{~mm}$ dia. and $80 \mathrm{~mm}$ length, Optifer

$\mathrm{p}_{\text {load }}=310^{3} \mathrm{~Pa}$, integration time checked $1 \mathrm{~ms}, 0.5 \mathrm{~ms}, 0.1 \mathrm{~ms}$ less than $1 / 1000$ deviation

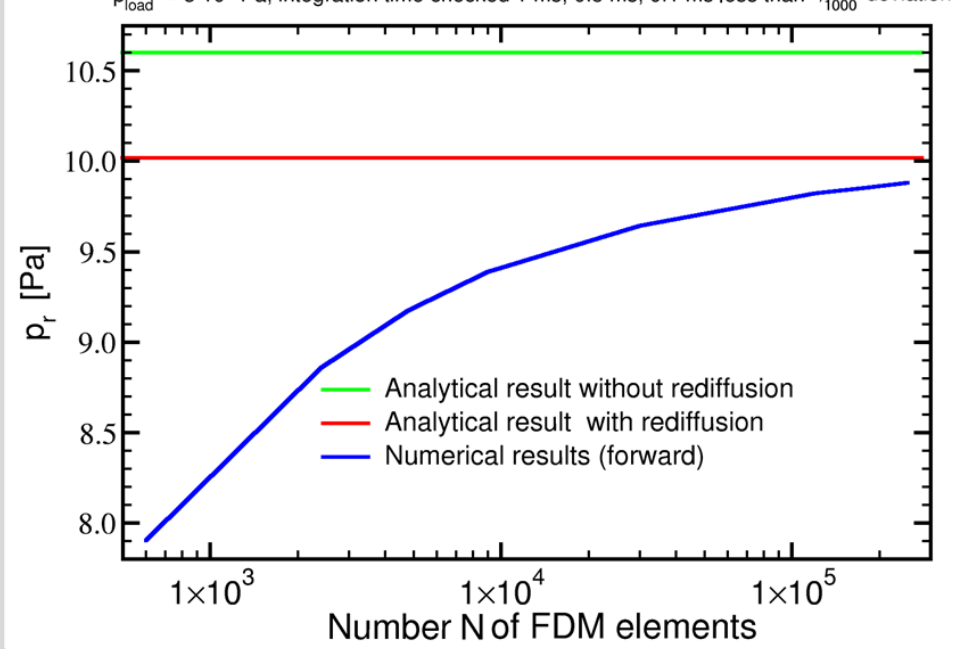

$10^{-11}<\frac{D_{s a} \Delta t}{\Delta z^{2}}<10^{-6}$

Results for chamber 40/20 mm dia. and $80 \mathrm{~mm}$ length, Optifer cylinder $60 \mathrm{~mm} \times 6 \mathrm{~mm}$ $\mathrm{p}_{\text {load }}=310^{3} \mathrm{~Pa}$, integration time $1 \mathrm{~ms}, 0.5 \mathrm{~ms}, 0.1 \mathrm{~ms}$ less than ${ }_{1}^{1}{ }_{1000}$ deviation

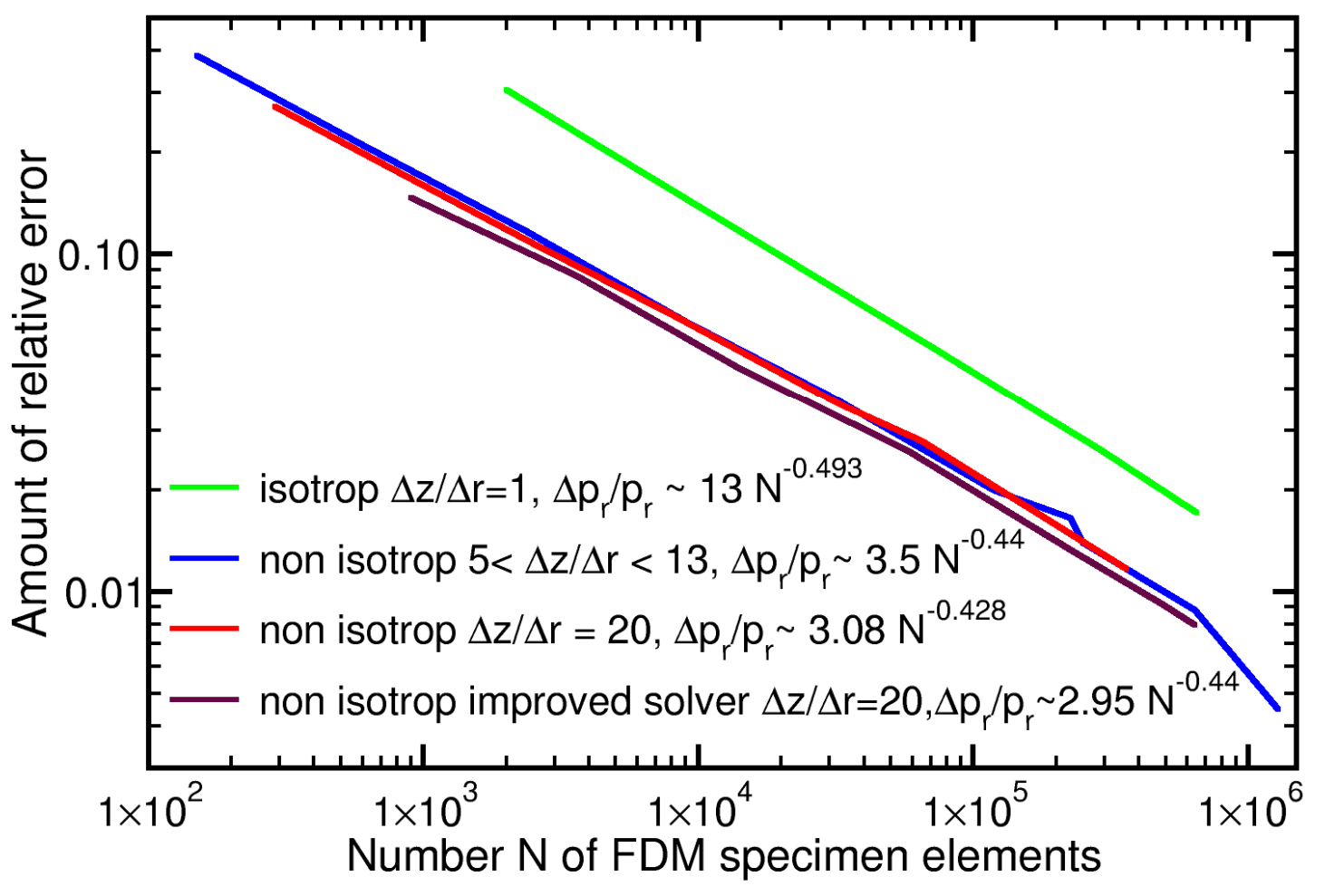


Small excurse to solver algorithms:

1D forward Euler

$$
\begin{gathered}
c(i, t+\Delta t)=c(i, t)+\frac{D \Delta t}{\Delta r^{2}}(c(i+1, t)+c(i-1, t)-2 c(i, t))+ \\
\frac{D \Delta t}{2 r \Delta r}((c(i+1, t))-c(i-1, t))
\end{gathered}
$$

2D improved forward Euler:

$$
\begin{aligned}
\mathrm{c}(i, j, t+\Delta t) & \\
=c(i, j, t) & +\frac{D \Delta t}{2 i \Delta r^{2}}((2 i+1) c(i+1, j, t)+(2 i-1) c(i-1, j, t)-(4 i) c(i, t)) \\
& +\frac{D \Delta t}{\Delta z^{2}}((c(i, j+1, t))+c(i, j-1, t)-2 c(i, j))
\end{aligned}
$$

Desired: Backward Euler solver, e. g. 1D cartesian:

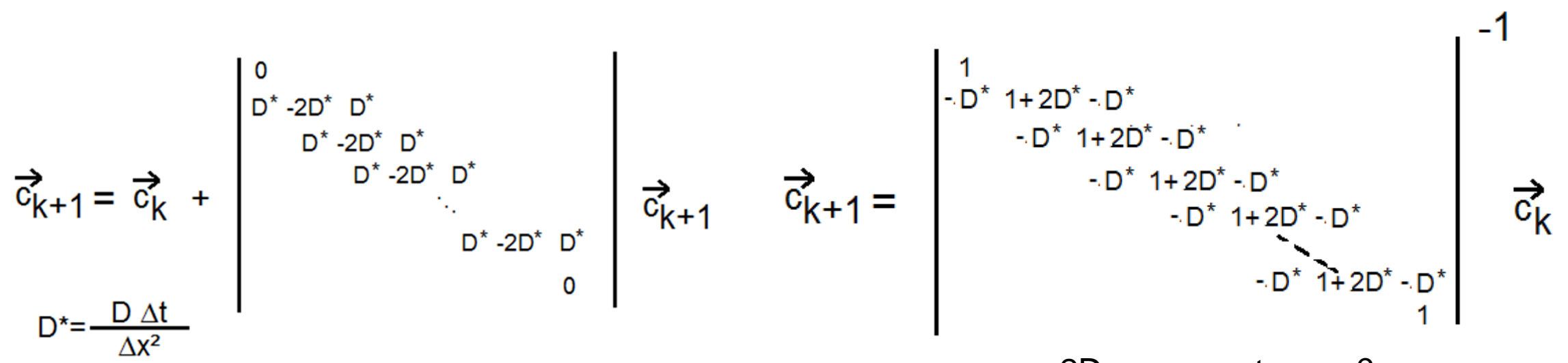

$2 \mathrm{D} \mathrm{n} \times \mathrm{n} \times \mathrm{m}$ tensor? 


\section{4.: Results}

"gasrel" calculation with 6400 specimen element and 1800 conf. elements 14 halftimes reaching endpresure $(0.1 \mathrm{~Pa})$

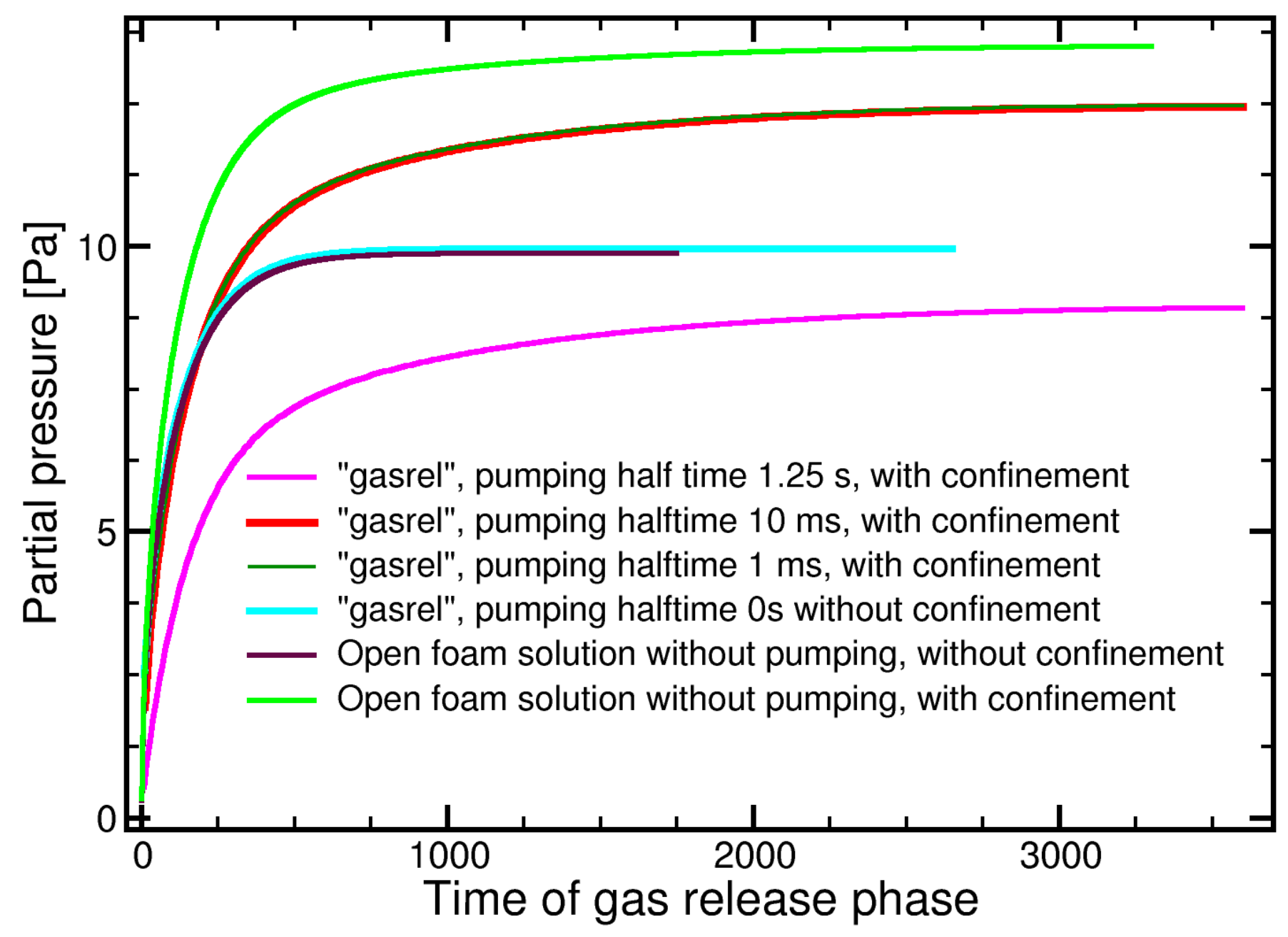


$\mathrm{T}=773 \mathrm{~K}, \mathrm{Cu}$ containment with Optifer specimen

chamber dimension $80 \mathrm{~mm}$ to $40 \mathrm{~mm}$ diameter, $p_{\text {load }}=310^{3} \mathrm{~Pa}$, interstitial-molecular

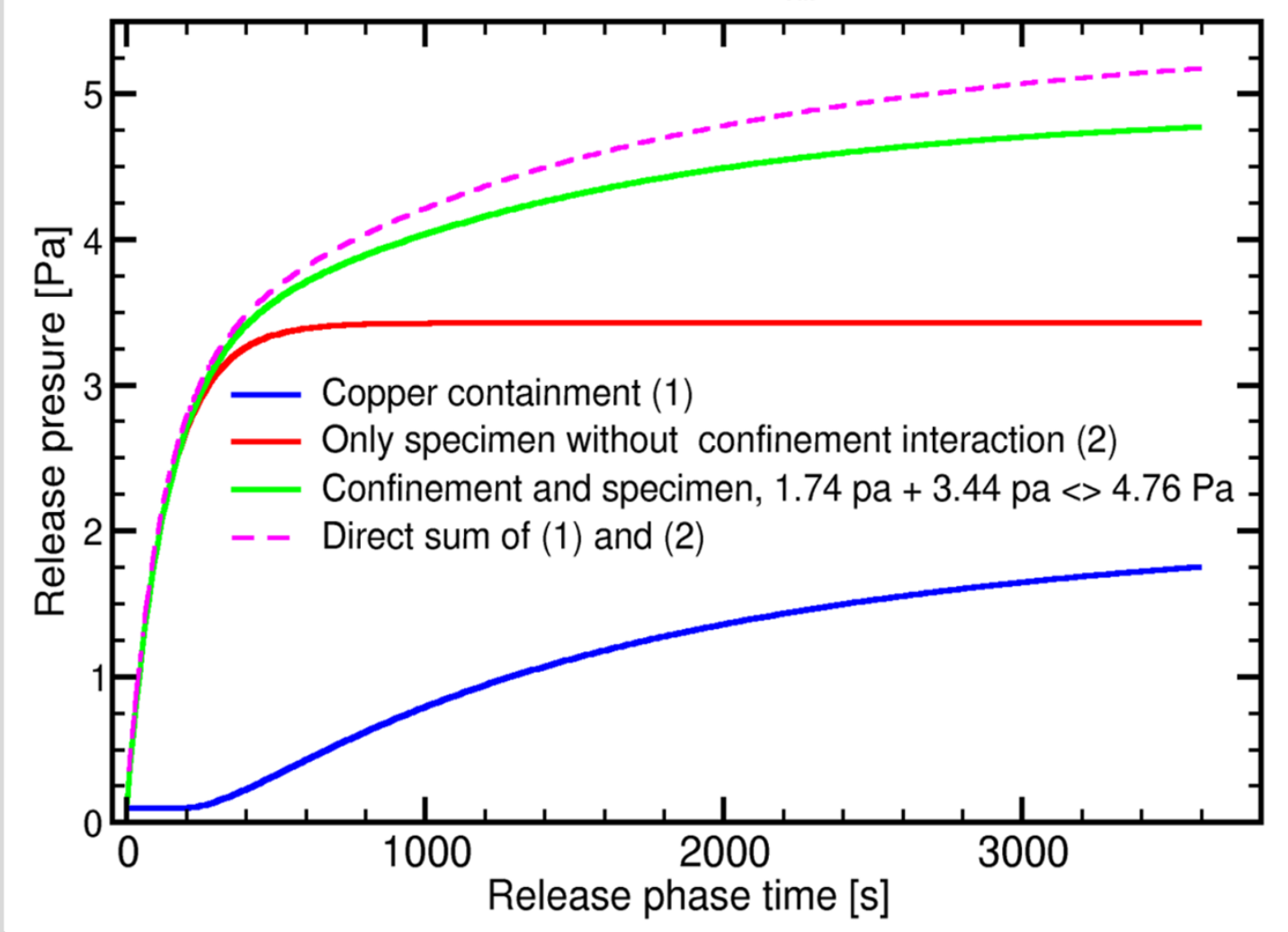

$\mathbb{N}$ CIT

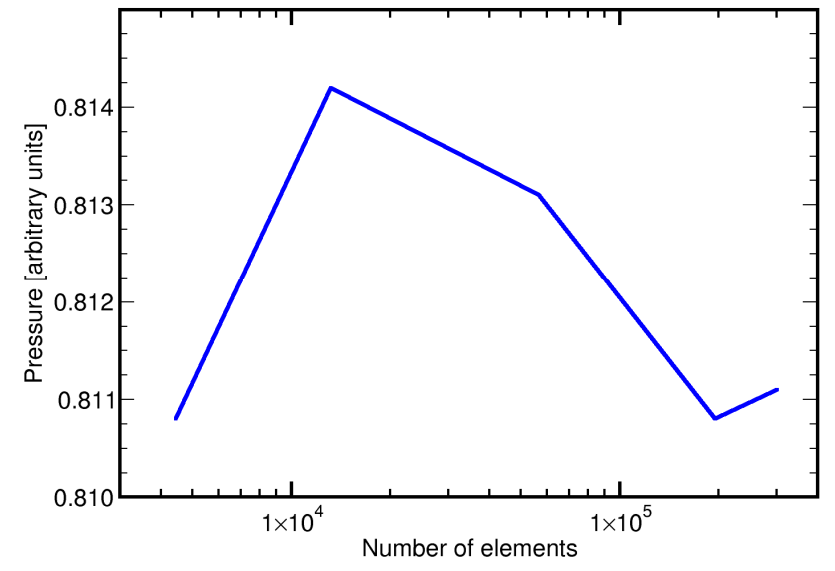

Choosing starting pressure:

Weak pump, half time $10 \mathrm{~s}$, total pressure $10^{5} \mathrm{Pas}, 3 \% \mathrm{D}_{2}$

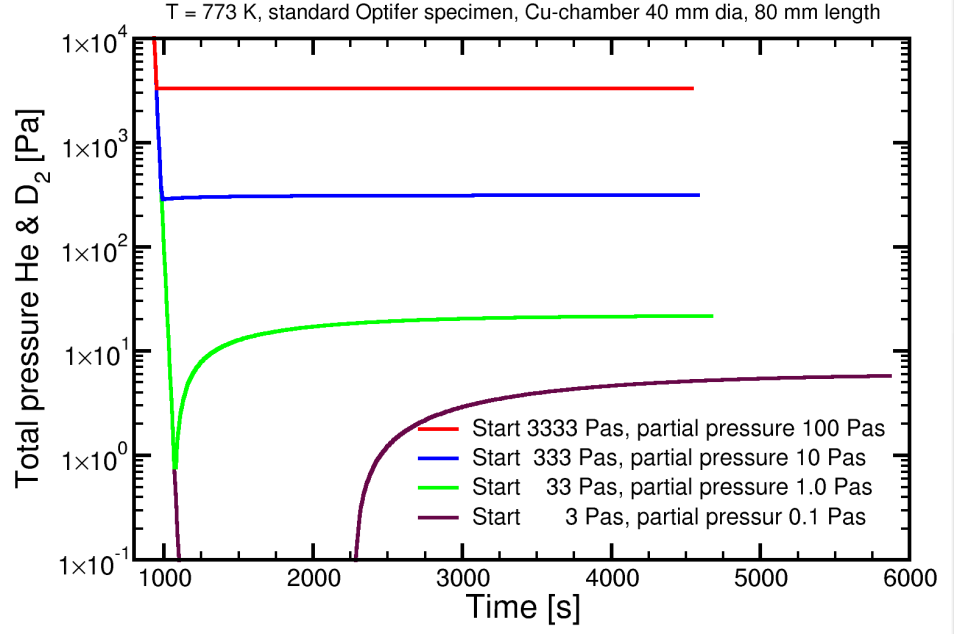




\section{Results for conception of experiments}

$20 \mathrm{~mm}$ diameter, pumping halftime $0.5 \mathrm{~s}, 773 \mathrm{~K}$ Total start pressure $33 \mathrm{Pas}$

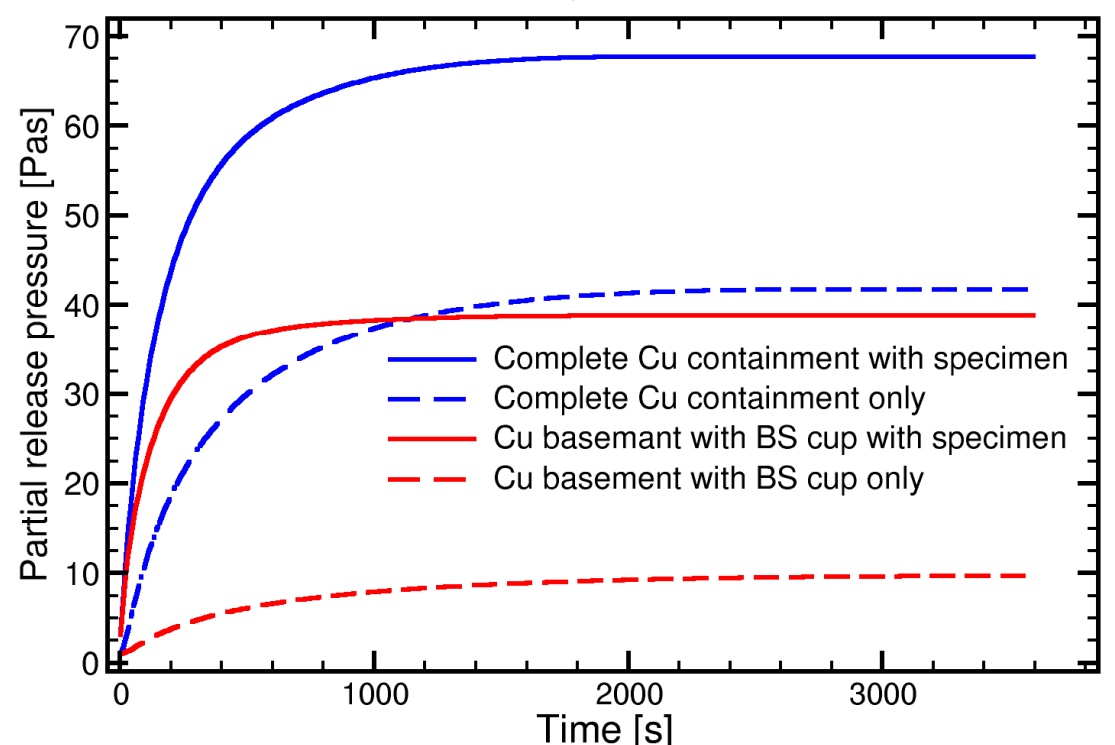

$40 \mathrm{~mm}$ diameter, pumping halftime $0.5 \mathrm{~s}, 773 \mathrm{~K}$

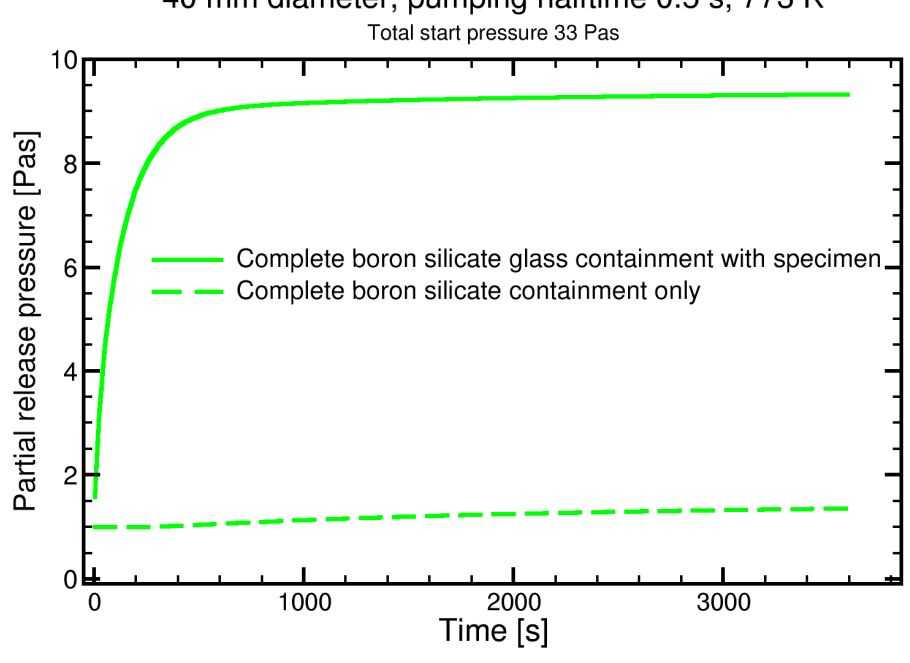

Copper or Boron Silicate glass

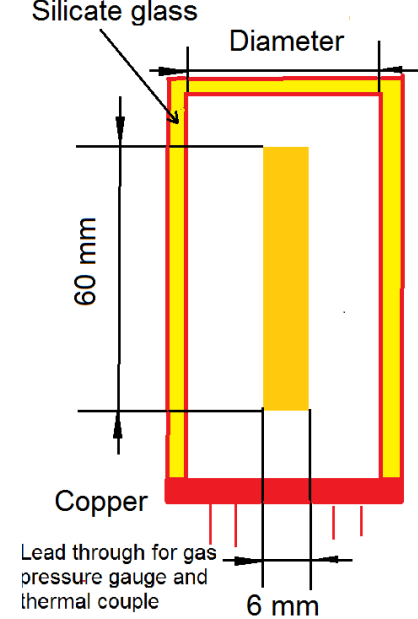

$40 \mathrm{~mm}$ diameter, pumping halftime $0.5 \mathrm{~s}, 773 \mathrm{~K}$ Total start pressure $33 \mathrm{Pas}$

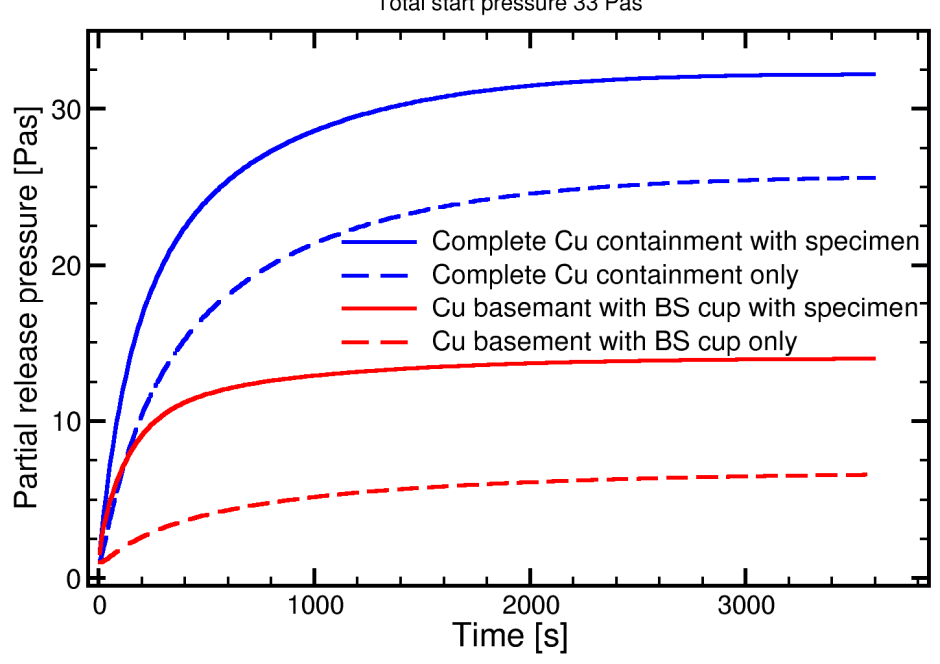

$80 \mathrm{~mm}$ diameter, pumping halftime $0.5 \mathrm{~s}, 773 \mathrm{~K}$

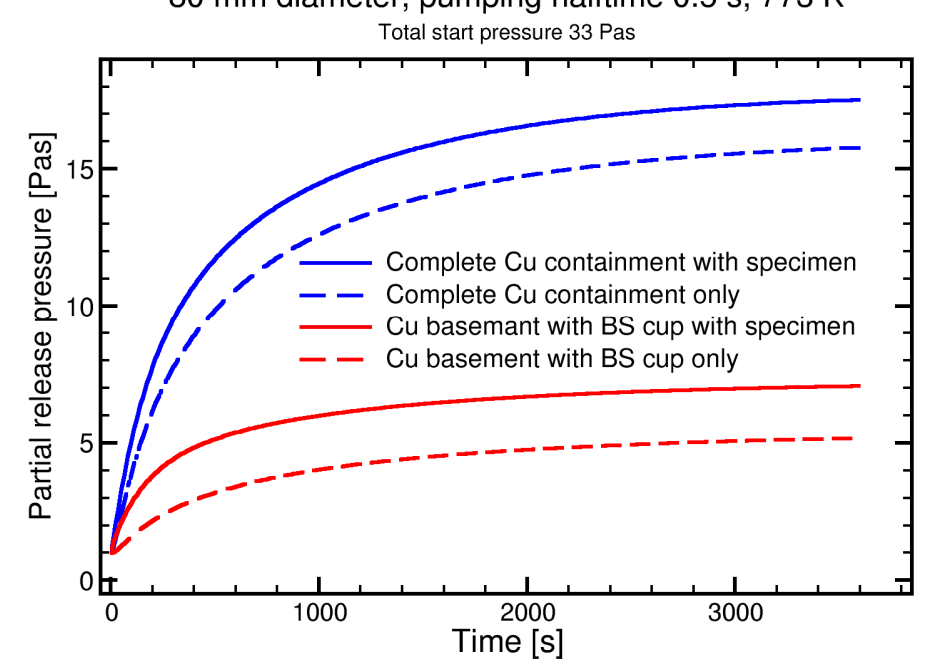




\section{5.: Outlook to analytical solution}

Solution in the charging interval

$c(r, z, t)=k_{s, s a} \sqrt{p_{\text {load }}} \sum_{n, m} \frac{8(-1)^{n+1}}{\pi(2 n+1) x_{m} J_{1}\left(x_{m}\right)} \exp \left(-\gamma_{n, m}^{2} t\right) \cos \left((2 n+1) \frac{\pi}{2} z\right) J_{0}\left(x_{m} r\right)$

where

$\gamma_{n, m}^{2}=D_{s a}\left(\frac{x_{m}^{2}}{r_{s}^{2}}+\frac{(2 n+1)^{2} \pi^{2}}{4 z_{s}^{2}}\right), J_{\alpha}(x)(\alpha=0,1)$ Bessel functions of the first kind,

$x_{m}$ the $m$-th roots of $J_{0}(x)$.
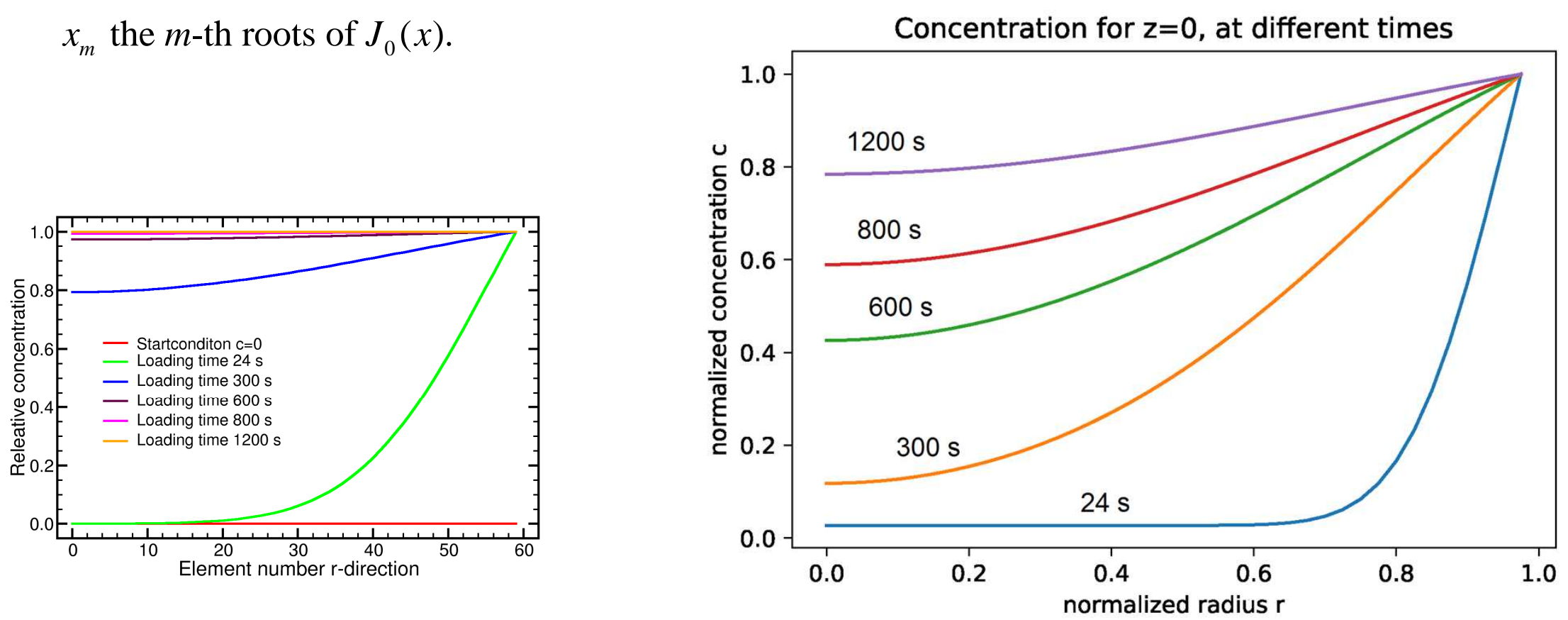


\title{
NUMERICAL ANALYSIS OF AN ISOVOLUMETRIC THERMAL DESORPTION EXPERIMENT
}

\author{
A. von der Weth, K. Nagatou,
}

F. Arbeiter, R. Dagan, D. Klimenko, V. Pasler, M. Schulz, INR, KIT, CN, Athens, 25th June, 2019

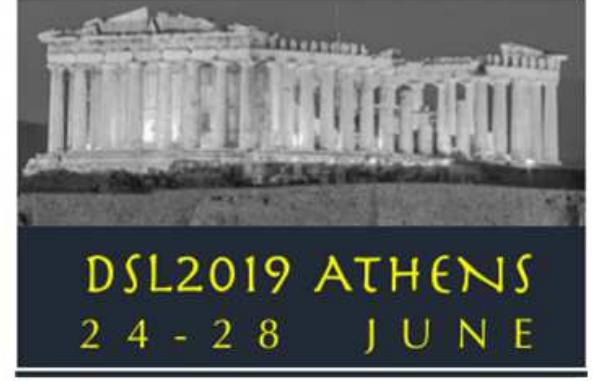

1.: Description of setup

2.: Simple analytical solutions

3.: Structure of Differential Equations

4.: Results of numerical optimization

5.: Outlook to analytical solution 


\section{Reminder: gas release diffusion experiments}

\section{Procedure:}

1. Specimen placed in defined volume enclusure

2. Load specimen with hydrogen at defined conditions $\left(T, \mathrm{p}_{\mathrm{H} 2}\right)$

3. Swiftly evacuate gases from enclosure

4. Measure pressure increase $p(t)$ in enclosure

$\rightarrow$ Sieverts' constant can be deduced from final pressure

$\rightarrow$ Diffusion constant can be deduced from pressure rise phase
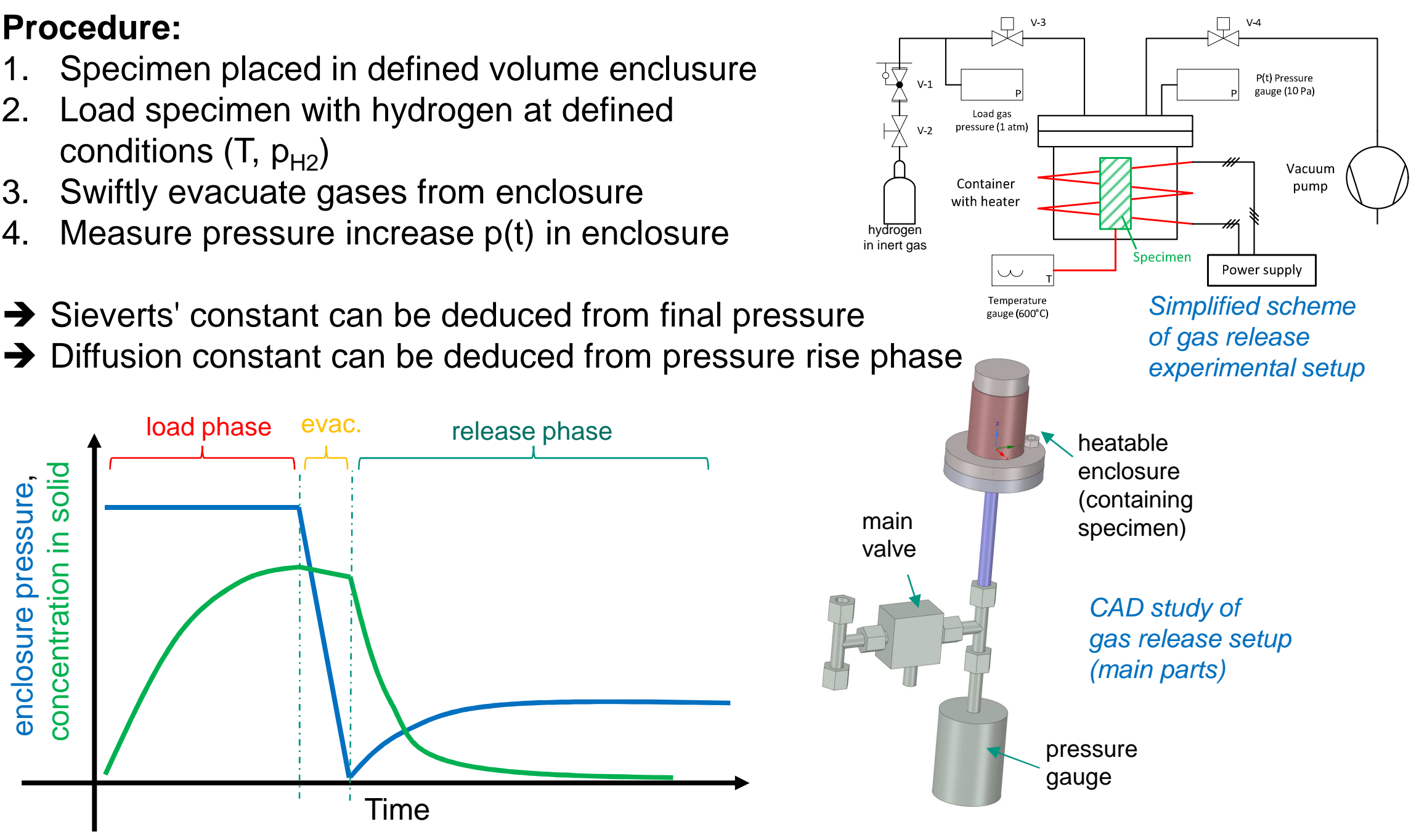


\section{1.: Description of setup of an Isovoluminetric Thermal}

Desorption Experiment

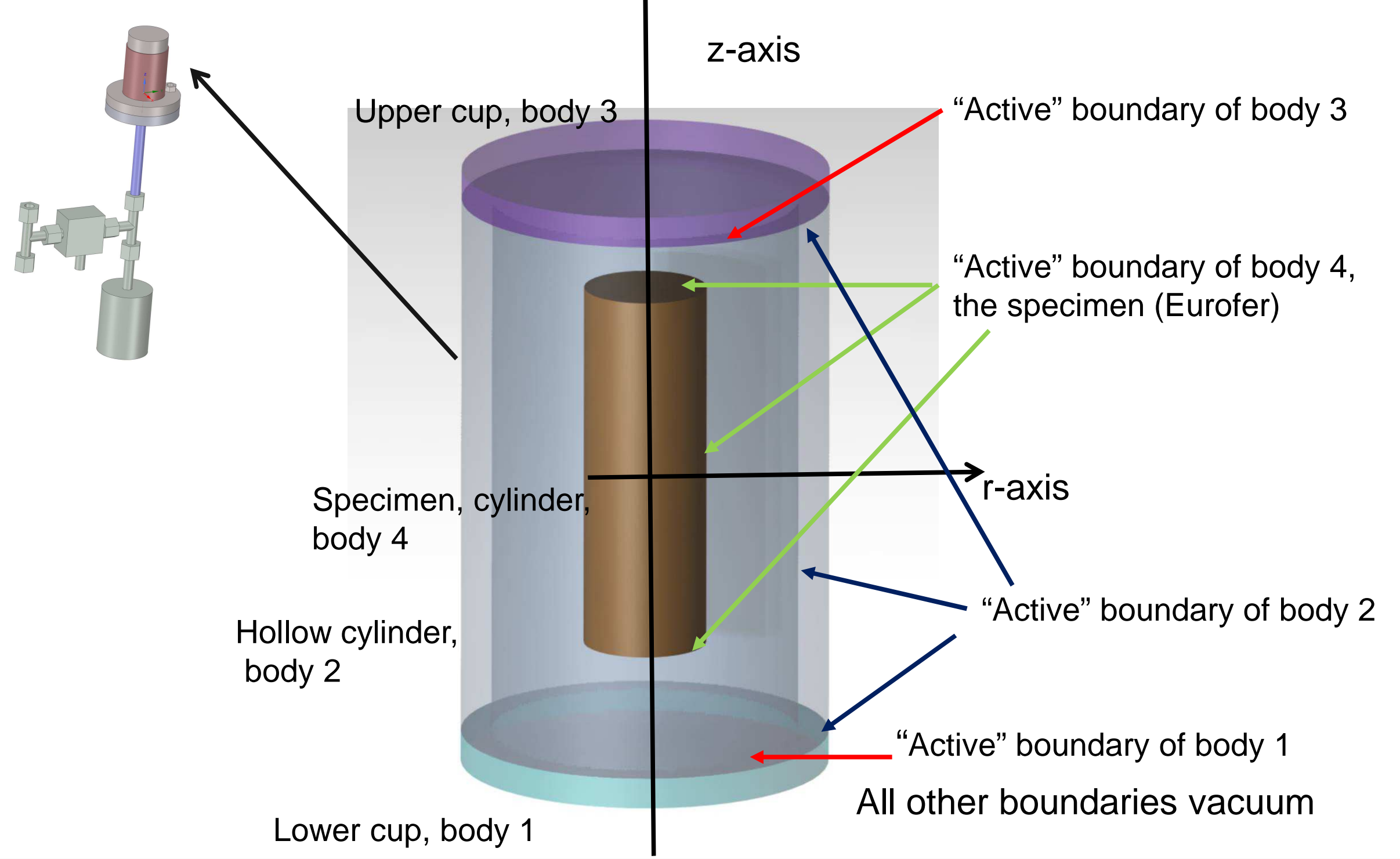




\section{1.: Physical description}

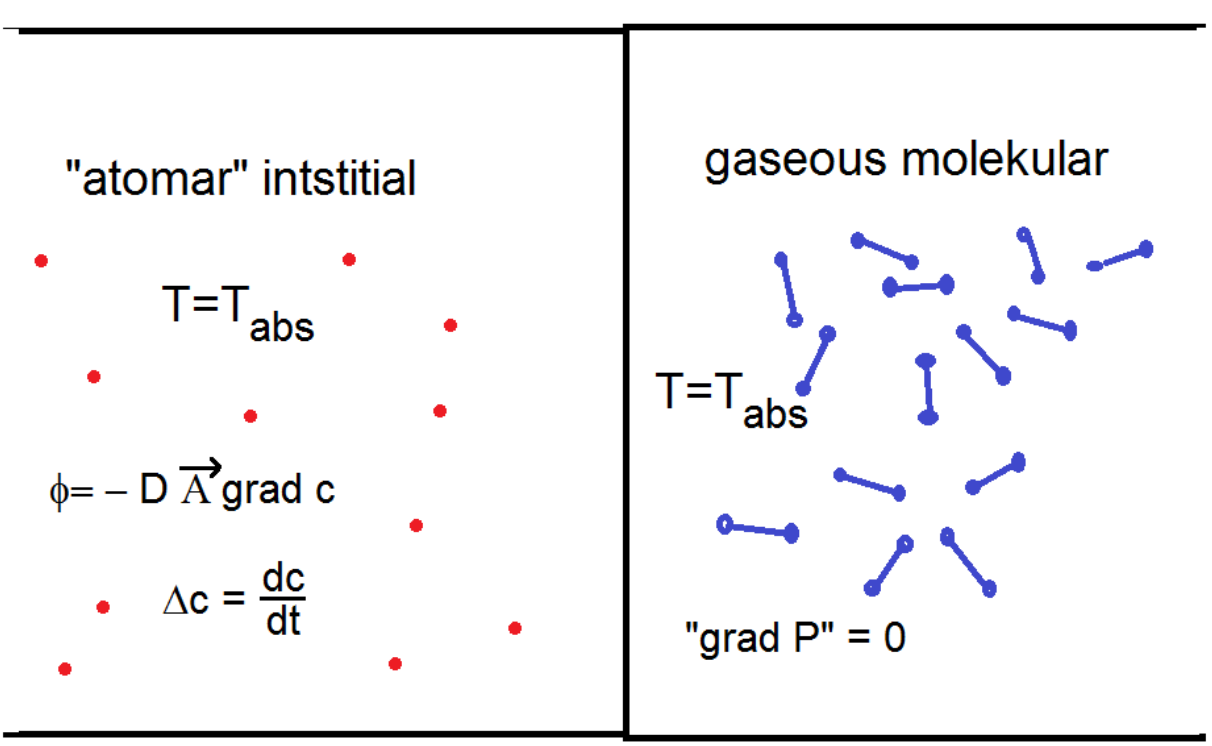

"Velocity" of mass transfer by diffusion constant D

Ratio of densities given by Sieverts' law (phase equilibrium)

$$
c=k_{s} \sqrt{p_{r}}
$$

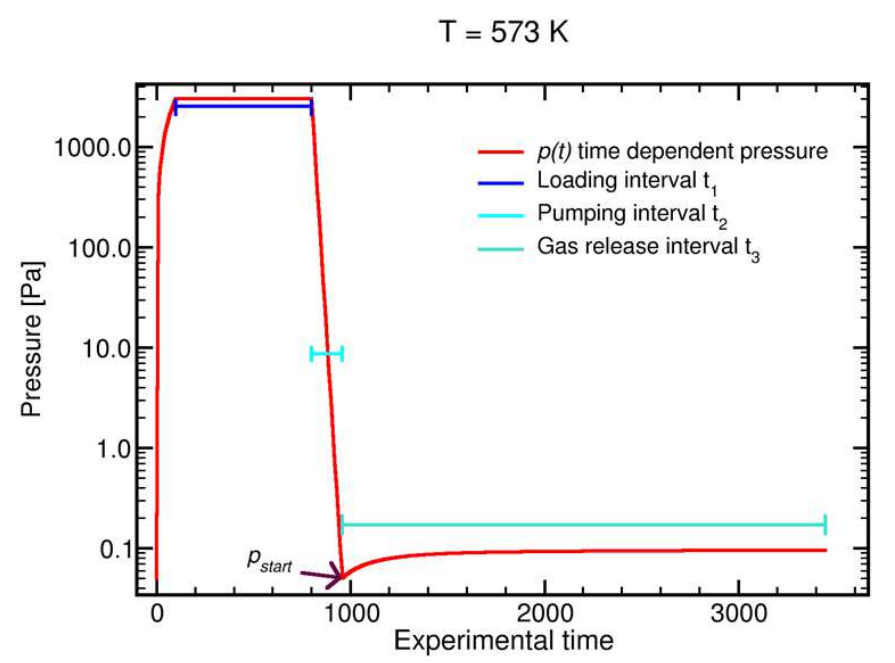

Removable cup

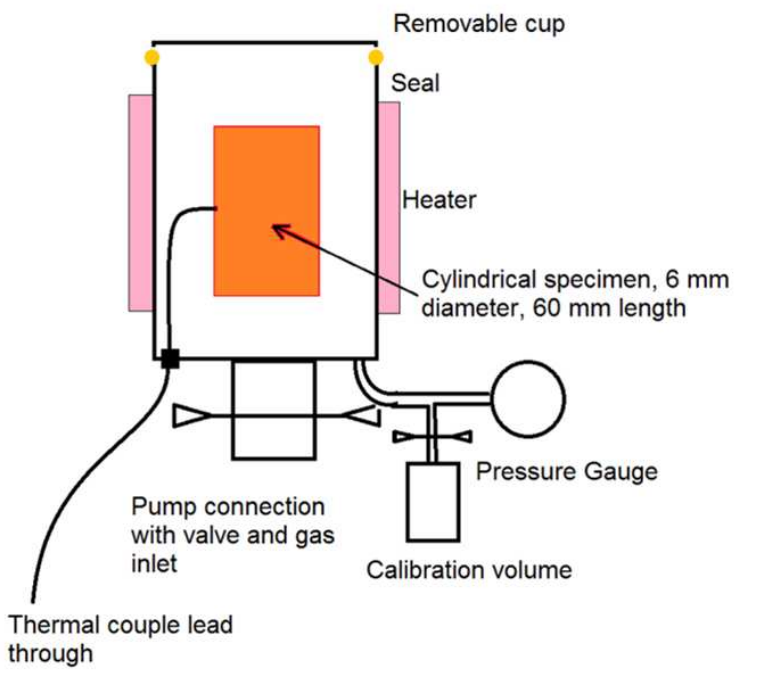




\section{2.: Simple analytical Solutions}

Without re-diffusion, complete outgassing, $c_{\text {sample }}=0$ :

$$
\underbrace{p_{r}}_{t \rightarrow \infty}=\underbrace{p_{\text {end }}}_{\begin{array}{c}
\text { switching off } \\
\text { pump }
\end{array}}+\frac{V_{s a} k_{s, s a} \sqrt{p_{\text {load }}} R T}{\begin{array}{c}
V_{c}-V_{\text {chamer }}-V_{\text {sample }} \\
\text { sample }
\end{array}}
$$

With phase equilibrium, mass conservation (number of "hydrogens" in atomic interstitial and molecular gaseous state constant) and non interacting confinement condition, currently unused:

$$
0=\frac{2\left(V_{c}-V_{s a}\right)}{R T}\left(\sqrt{p_{r}}\right)^{2}+V_{s} k_{s, s a} \underbrace{\sqrt{p_{r}}}_{=" x^{\prime \prime}}-\left(V_{s} k_{s, s a} \sqrt{p_{\text {load }}}+\frac{2 p_{\text {end }}\left(V_{c}-V_{s a}\right)}{R T}\right)
$$

$$
k_{s, s a}=\frac{2\left(V_{c}-V_{s a}\right)}{R T V_{s a}} \frac{\left(p_{r}-p_{\text {end }}\right)}{\sqrt{p_{\text {load }}-\sqrt{p_{r}}}}
$$

Experimentally difficult realization of boundaries, no statement about diffusion constant

$$
\underbrace{p_{r}}_{t \rightarrow \infty}=\left(\frac{-1 \underbrace{+}_{n e g . s q r t} \sqrt{1+\left(\frac{8\left(V_{c}-V_{s a}\right) \sqrt{p_{\text {load }}}}{R T k_{s, s a}}+\frac{16 p_{\text {end }}\left(V_{c}-V_{s a}\right)^{2}}{\left(R T V_{s a} k_{s, s a}\right)^{2}}\right)}}{\left(\frac{V_{s a} k_{s, s a} R T}{4\left(V_{c}-V_{s a}\right)}\right)^{-1}}\right)^{2}
$$




\section{3.: Structure of differential equations:}

$$
\begin{aligned}
& \frac{\partial c}{\partial t}=D_{s a} \Delta c \quad \frac{\partial d(i)}{\partial t}=D_{c u} \Delta d(i), i=1,2,3 \\
& \Delta=\frac{\partial^{2}}{\partial r^{2}}+\frac{1}{r} \frac{\partial}{\partial r}+\frac{\partial^{2}}{\partial z^{2}}+\underbrace{\frac{1}{r^{2}} \frac{\partial^{2}}{\partial \varphi^{2}}}_{=0} \\
& c\left(0 \leq r \leq r_{s}, z= \pm z_{s}, \forall t\right)=k_{s, s a} \sqrt{p(t)} \\
& c\left(r=r_{s},|z| \leq z_{s}, \forall t\right)=k_{s, s a} \sqrt{p(t)} \\
& d(1)\left(r \leq r_{c o}, z=-z_{c i}, \forall t\right)=k_{s, c u} \sqrt{p(t)} \\
& d(2)\left(r=r_{c i},-z_{c i} \leq z \leq z_{c i}, \forall t\right)=k_{s, c u} \sqrt{p(t)} \\
& d(2)\left(r_{c i} \leq r \leq r_{c o}, z= \pm z_{c i}, \forall t\right)=k_{s, c u} \sqrt{p(t)} \\
& d(3)\left(r \leq r_{c o}, z=z_{c i}, \forall t\right)=k_{s, c u} \sqrt{p(t)}
\end{aligned}
$$

Analytical 1d: Sedano, Perujo (1999), Esteban Douglas (2001) , Eichenauer Pebler (1957) Hattenbach (1961)

Analytical 2d: Eichenauer, Pebler, Witte 1965

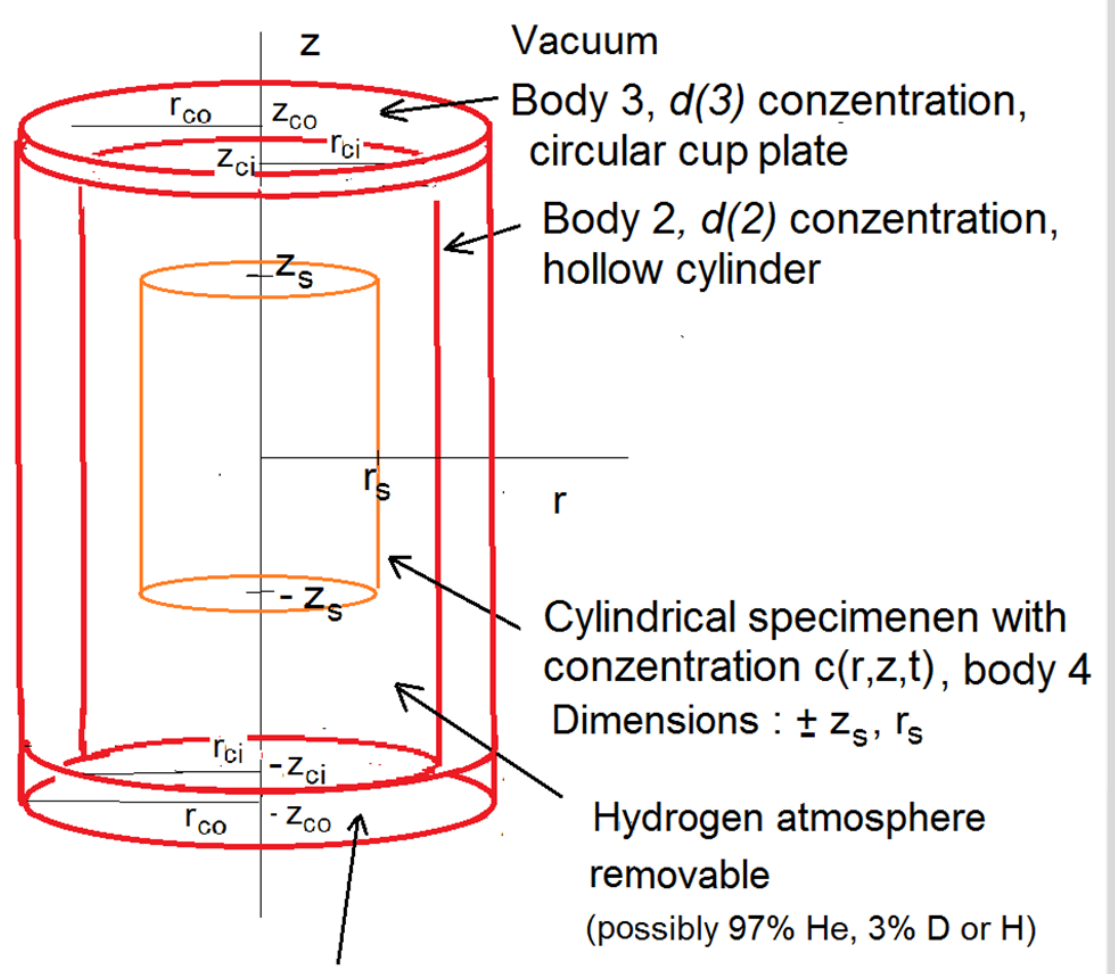

Body 1, $d(1)$ conzentration 


\section{Structure of differential equations}

$$
\frac{d m}{d t}=-2 \pi D_{s a} \int_{0}^{r_{s}} \underbrace{2}_{\text {symmetric }} r \frac{\partial}{\partial z} c\left(r, z=z_{s}, t\right) d r
$$

- $\underbrace{2 \pi r_{s} D_{s a} \int_{-z_{s}}^{z_{s}} \frac{\partial}{\partial r} c\left(r=r_{s}, z, t\right) d z}_{\text {superficies surface }}$

superficies surface of specimen

- $\underbrace{2 \pi D_{c u} \int_{0}^{r_{c o}} r \frac{\partial}{\partial z} d(1)\left(r, z=-z_{c i}, t\right) d r}$ circular aerea of body 1

- $2 \pi r_{c i} D_{c u} \int_{-z_{c i}}^{z_{c i}} \frac{\partial}{\partial r} d(2)\left(r=r_{c i}, z, t\right) d z$ superficies surface of body 2 $-\underbrace{2 \pi D_{c u} \int_{0}^{r_{c o}} r \frac{\partial}{\partial z} d(3)\left(r, z=z_{c i}, t\right) d r}_{\text {circular area of body } 3}$

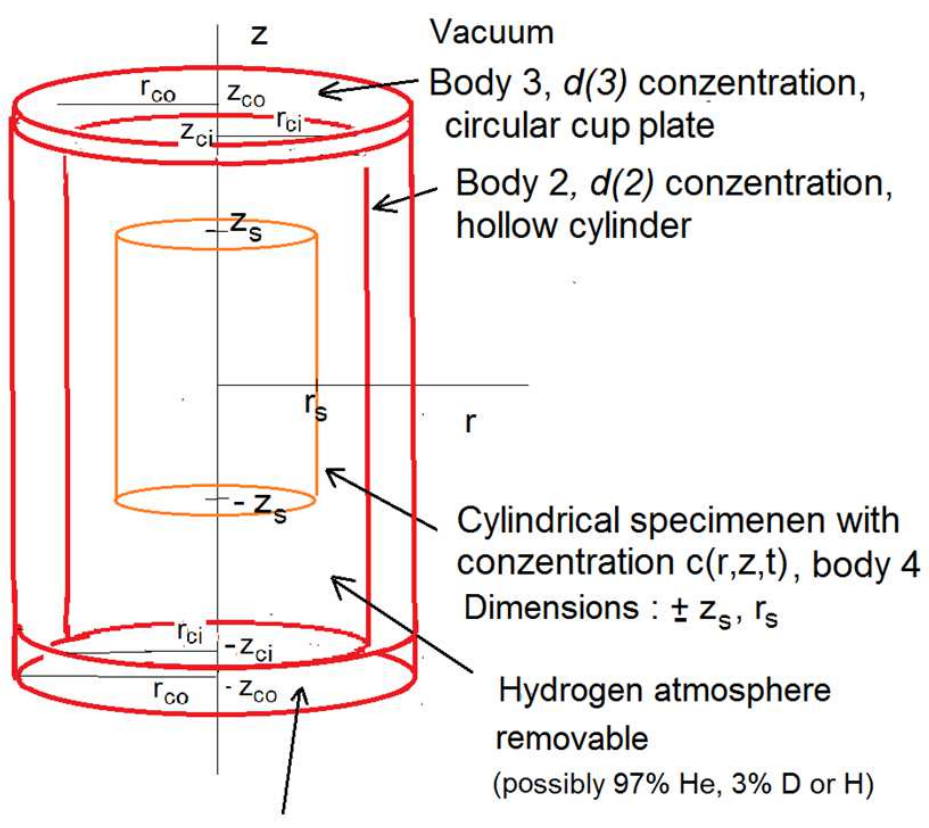

Body 1, d(1) conzentration

$$
p(t)=p_{\text {start }}+\underbrace{k_{v}}_{R T_{\text {abs }} / V_{\text {gas }}} \int_{t_{1}+t_{2}}^{t} \underbrace{0.5}_{\begin{array}{c}
\text { gaseous } \\
\text { interstitial }
\end{array}} \frac{d m}{d t} d t
$$




\section{4.: Results of numerical solution}

$\mathrm{T}=773 \mathrm{~K}, \mathrm{Cu}$ containment with Optifer specimen, 13. 5. chamber dimension $80 \mathrm{~mm}$ to $40 \mathrm{~mm}$ diameter, $\mathrm{p}_{\text {load }}=310^{3} \mathrm{~Pa}$, improved gradient calculation

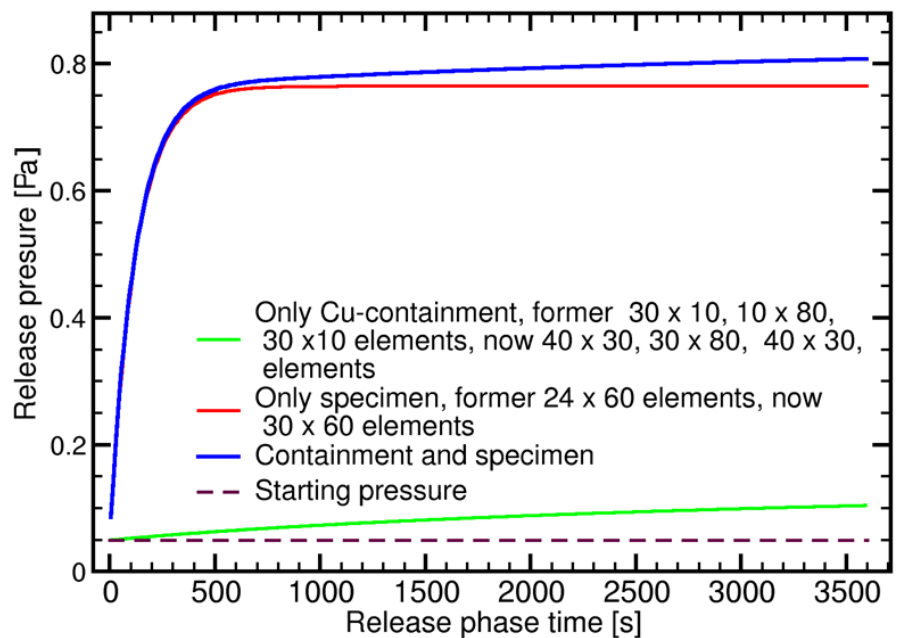

Simple numerical gradient calculation

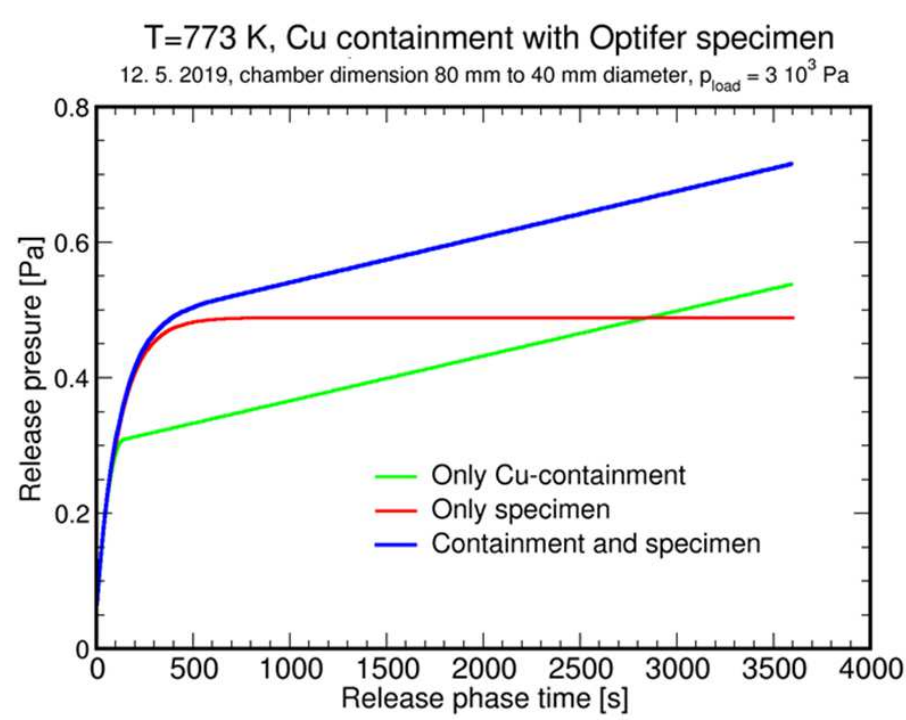

\section{Numerical artefacts}

Development, $\mathrm{T}=773 \mathrm{~K}$, Cu cont. with Optifer specimen chamber dimension $80 \mathrm{~mm}$ to $40 \mathrm{~mm}$ diameter, $\mathrm{p}_{\text {load }}=310^{3} \mathrm{~Pa}$, improved gradient calculation

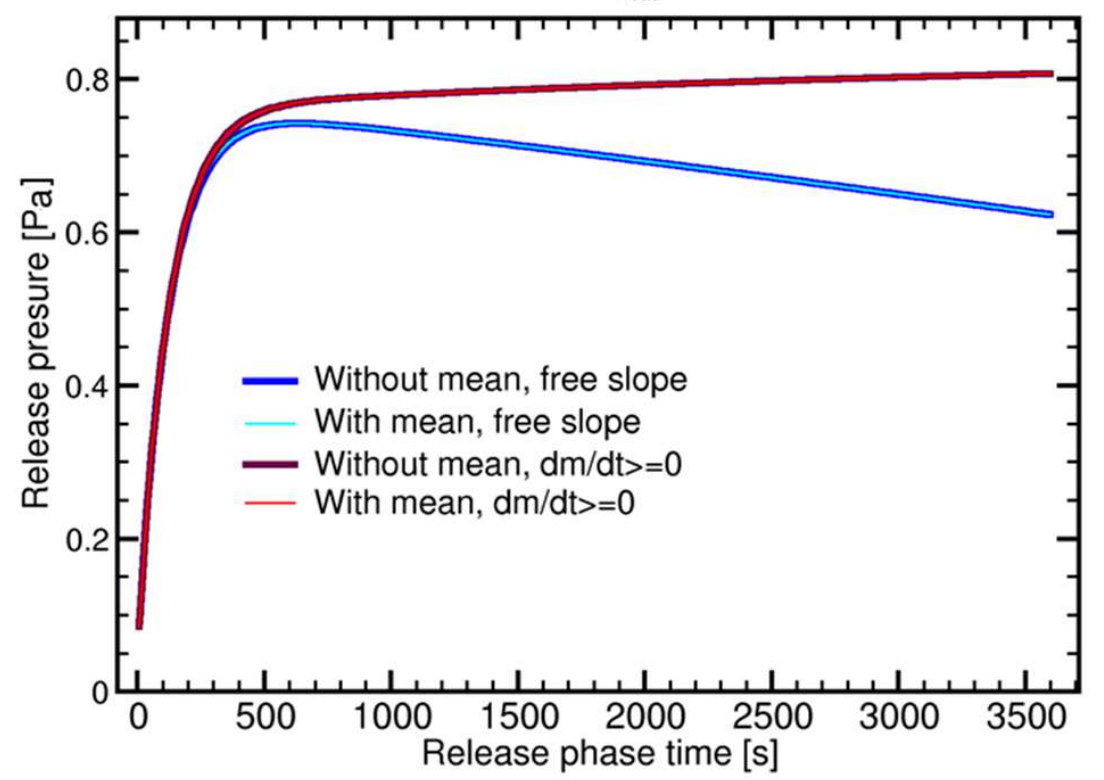




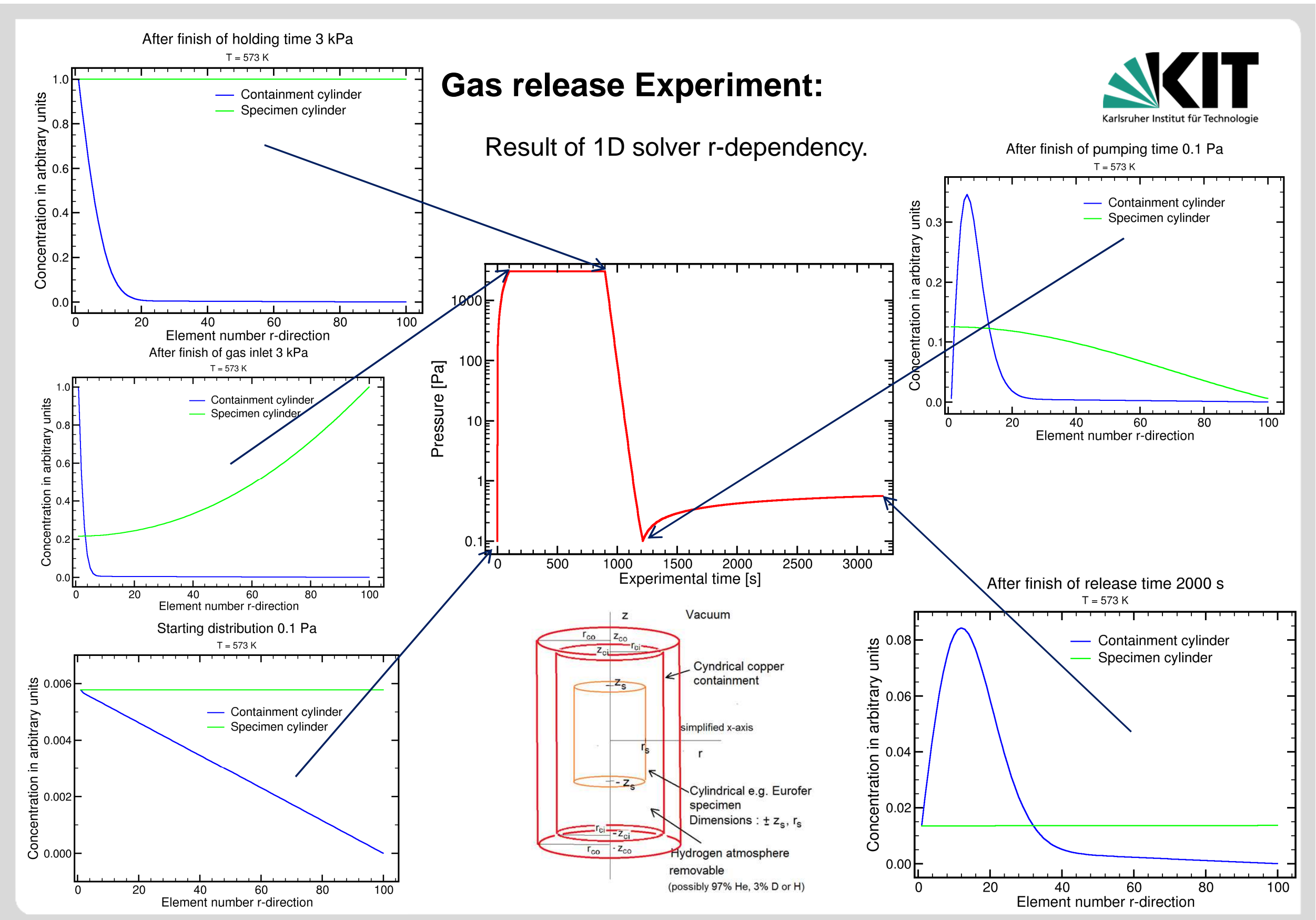

$925^{\text {th }}$ of June, NUMERICAL ANALYSIS OF AN ISOVOLUMETRIC THERMAL DESORPTION EXPERIMENT

MET/INR/Maschinenbau 


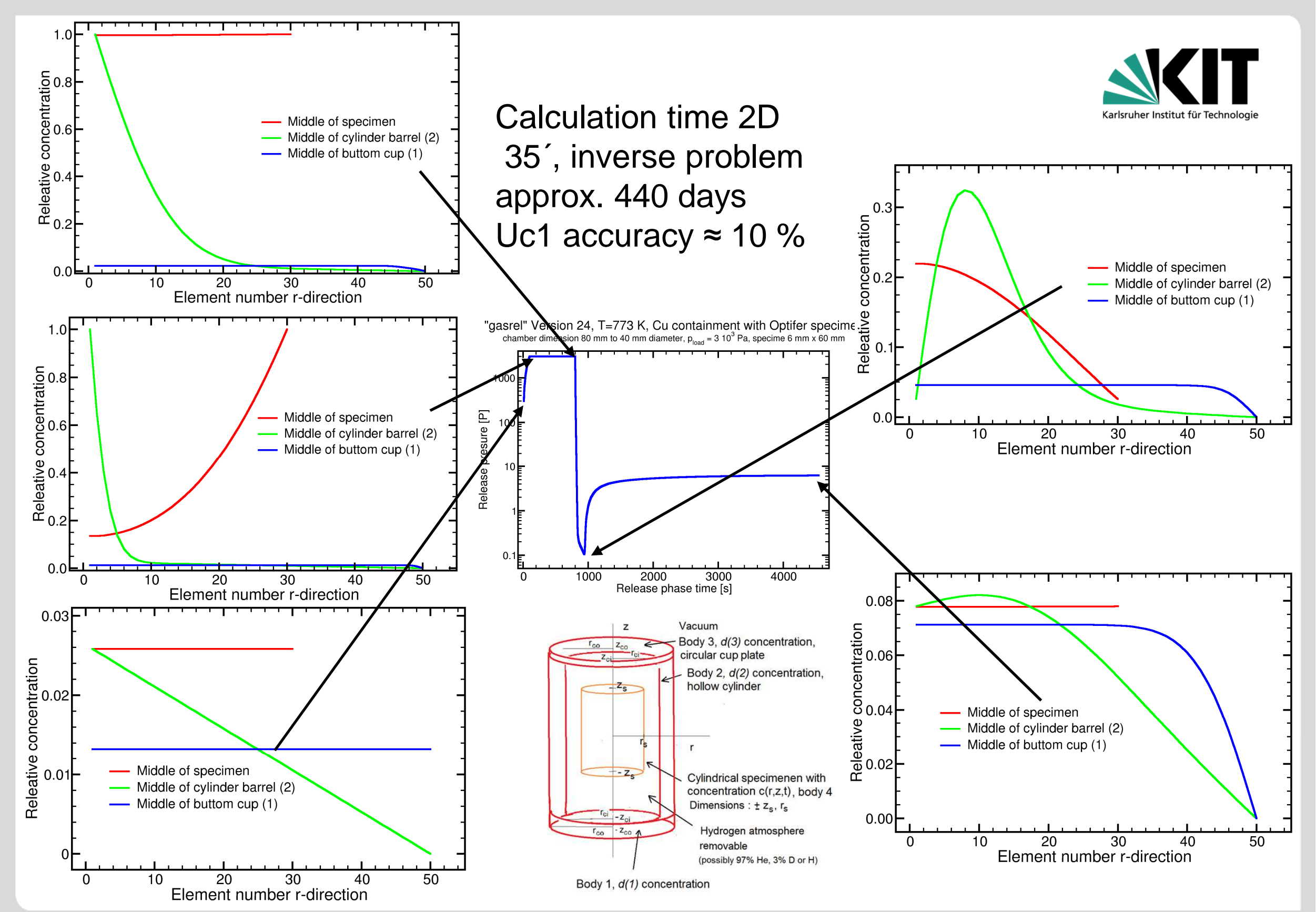




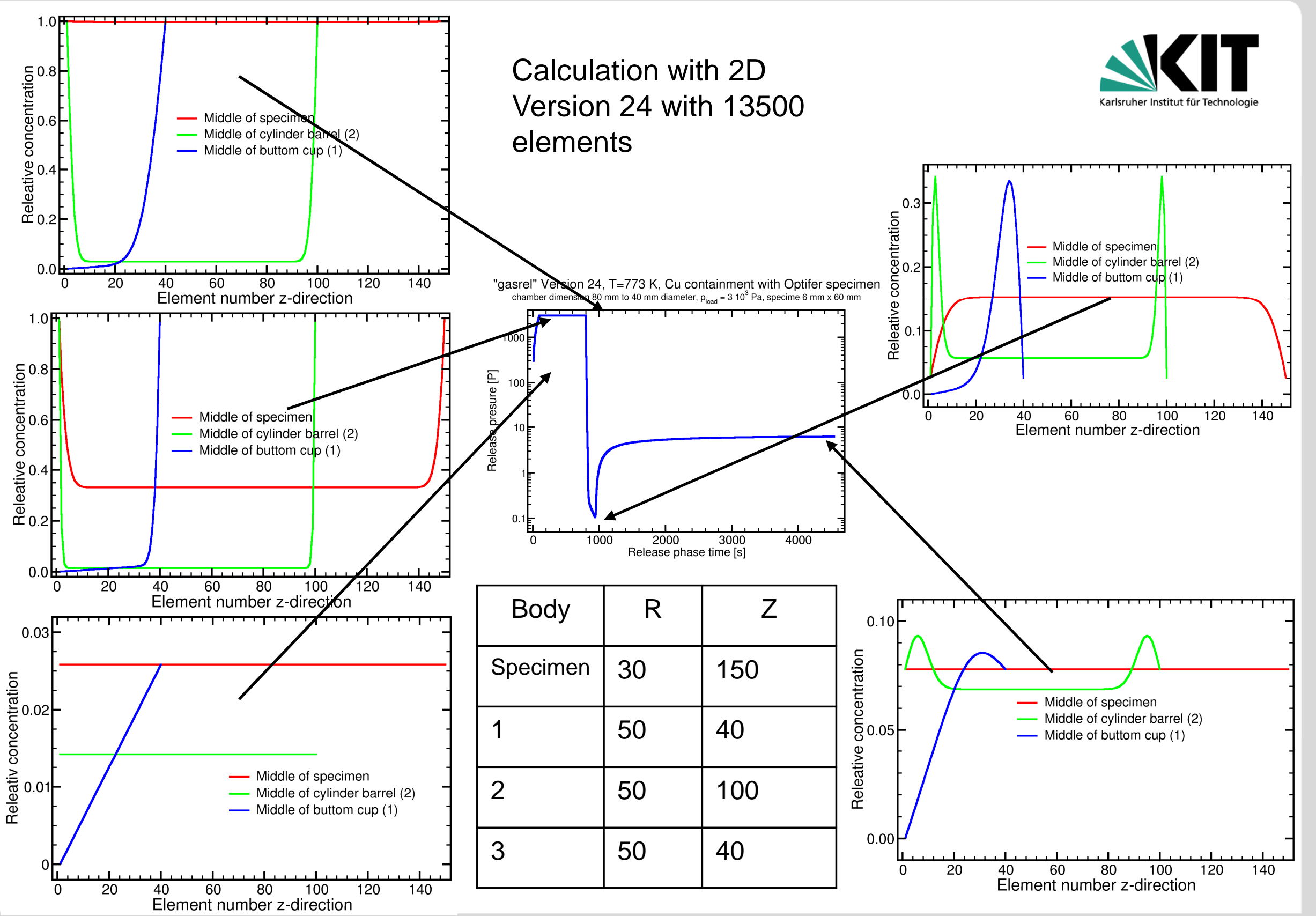

$1125^{\text {th }}$ of June, NUMERICAL ANALYSIS OF AN ISOVOLUMETRIC THERMAL DESORPTION EXPERIMENT

MET/INR/Maschinenbau 


\section{Comparison with analytical solution:}

Results for chamber $40 \mathrm{~mm}$ dia. and $80 \mathrm{~mm}$ length, Optifer

$\mathrm{p}_{\text {load }}=310^{3} \mathrm{~Pa}$, integration time checked $1 \mathrm{~ms}, 0.5 \mathrm{~ms}, 0.1 \mathrm{~ms}$ less than $1 / 1000$ deviation

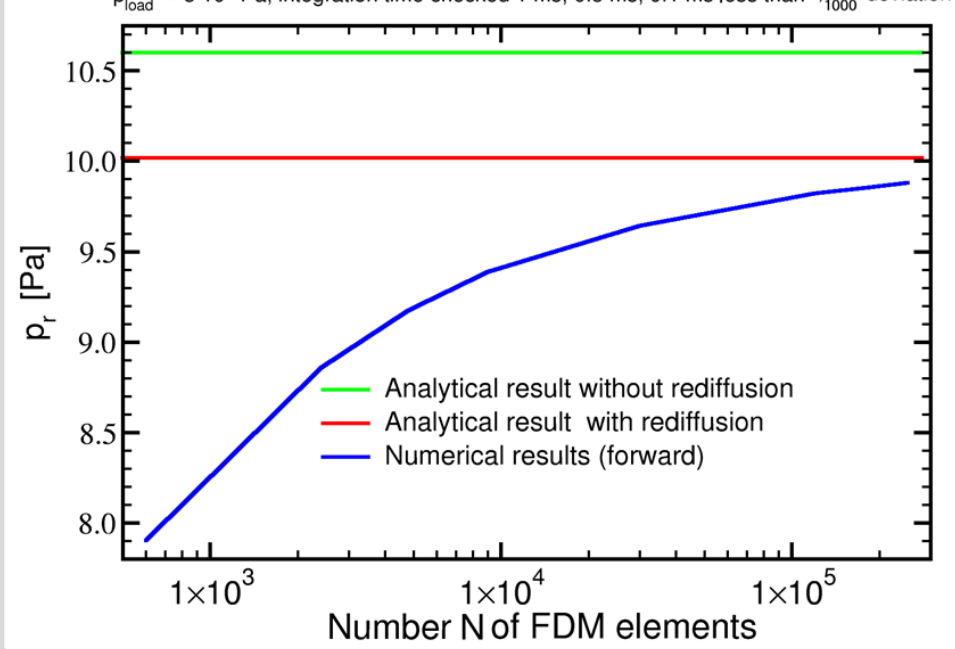

$10^{-11}<\frac{D_{s a} \Delta t}{\Delta z^{2}}<10^{-6}$

Results for chamber 40/20 mm dia. and $80 \mathrm{~mm}$ length, Optifer cylinder $60 \mathrm{~mm} \times 6 \mathrm{~mm}$ $\mathrm{p}_{\text {load }}=310^{3} \mathrm{~Pa}$, integration time $1 \mathrm{~ms}, 0.5 \mathrm{~ms}, 0.1 \mathrm{~ms}$ less than ${ }_{1}^{1}{ }_{1000}$ deviation

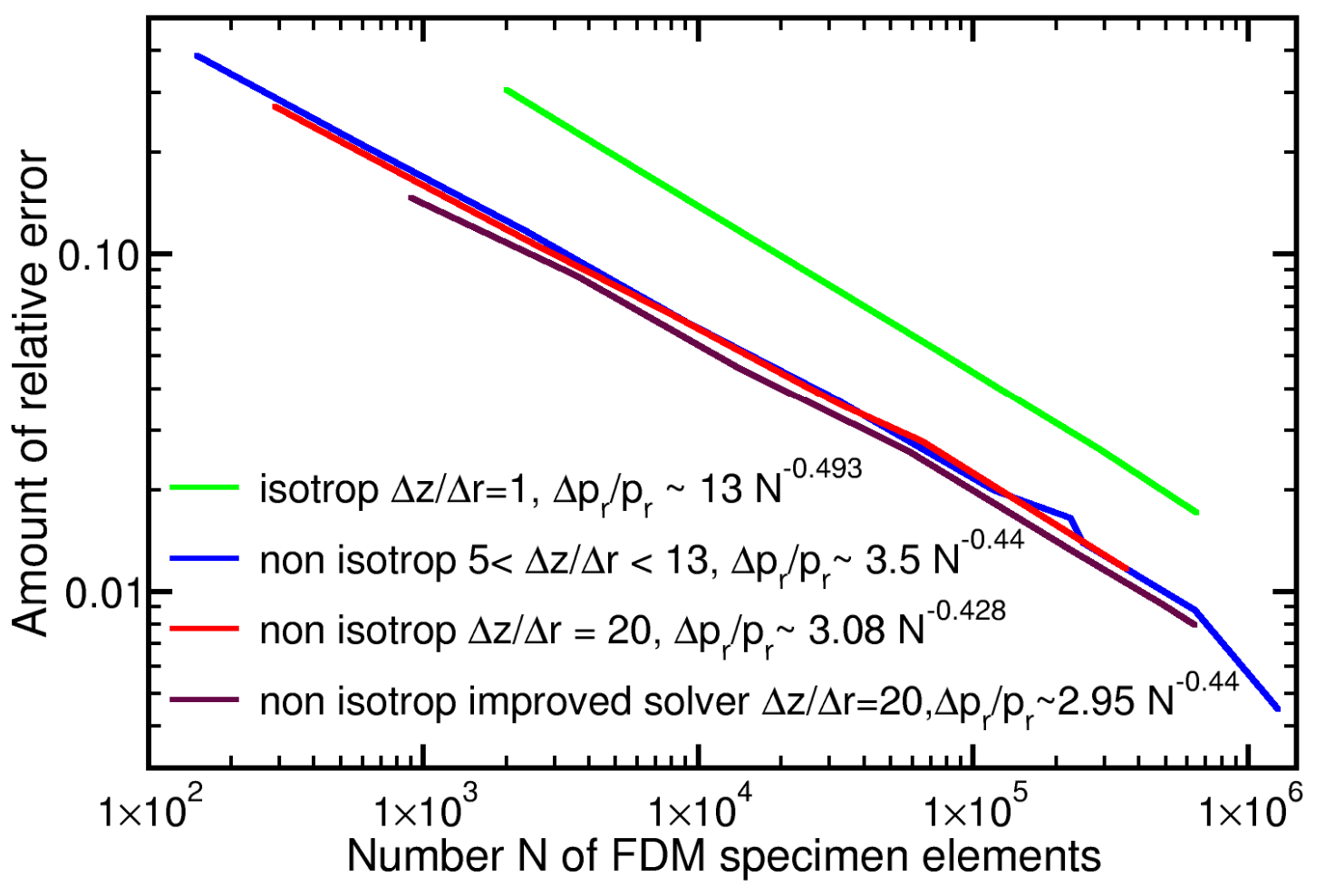


Small excurse to solver algorithms:

1D forward Euler

$$
\begin{gathered}
c(i, t+\Delta t)=c(i, t)+\frac{D \Delta t}{\Delta r^{2}}(c(i+1, t)+c(i-1, t)-2 c(i, t))+ \\
\frac{D \Delta t}{2 r \Delta r}((c(i+1, t))-c(i-1, t))
\end{gathered}
$$

2D improved forward Euler:

$$
\begin{aligned}
\mathrm{c}(i, j, t+\Delta t) & \\
=c(i, j, t) & +\frac{D \Delta t}{2 i \Delta r^{2}}((2 i+1) c(i+1, j, t)+(2 i-1) c(i-1, j, t)-(4 i) c(i, t)) \\
& +\frac{D \Delta t}{\Delta z^{2}}((c(i, j+1, t))+c(i, j-1, t)-2 c(i, j))
\end{aligned}
$$

Desired: Backward Euler solver, e. g. 1D cartesian:

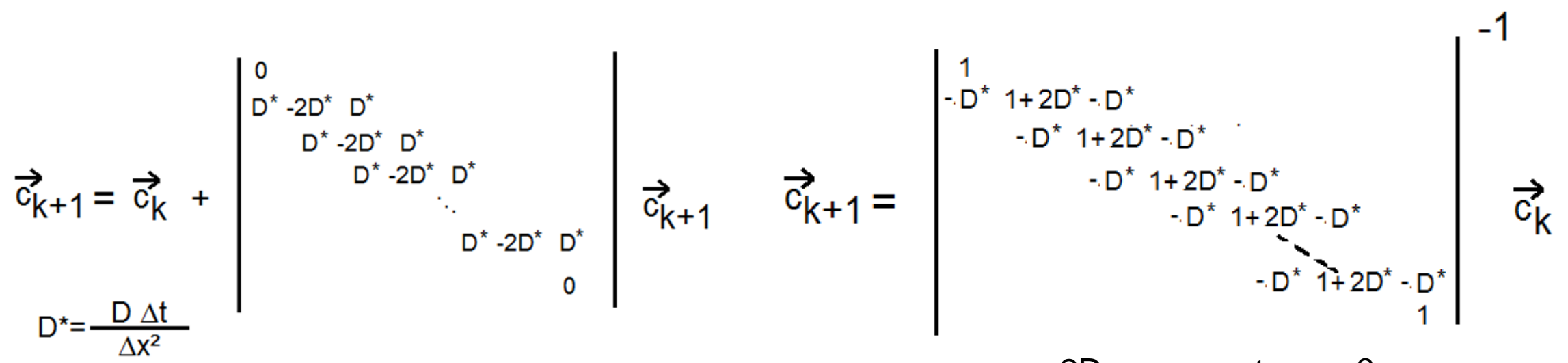

$2 \mathrm{D} \mathrm{n} \times \mathrm{n} \times \mathrm{m}$ tensor? 


\section{4.: Results}

"gasrel" calculation with 6400 specimen element and 1800 conf. elements 14 halftimes reaching endpresure $(0.1 \mathrm{~Pa})$

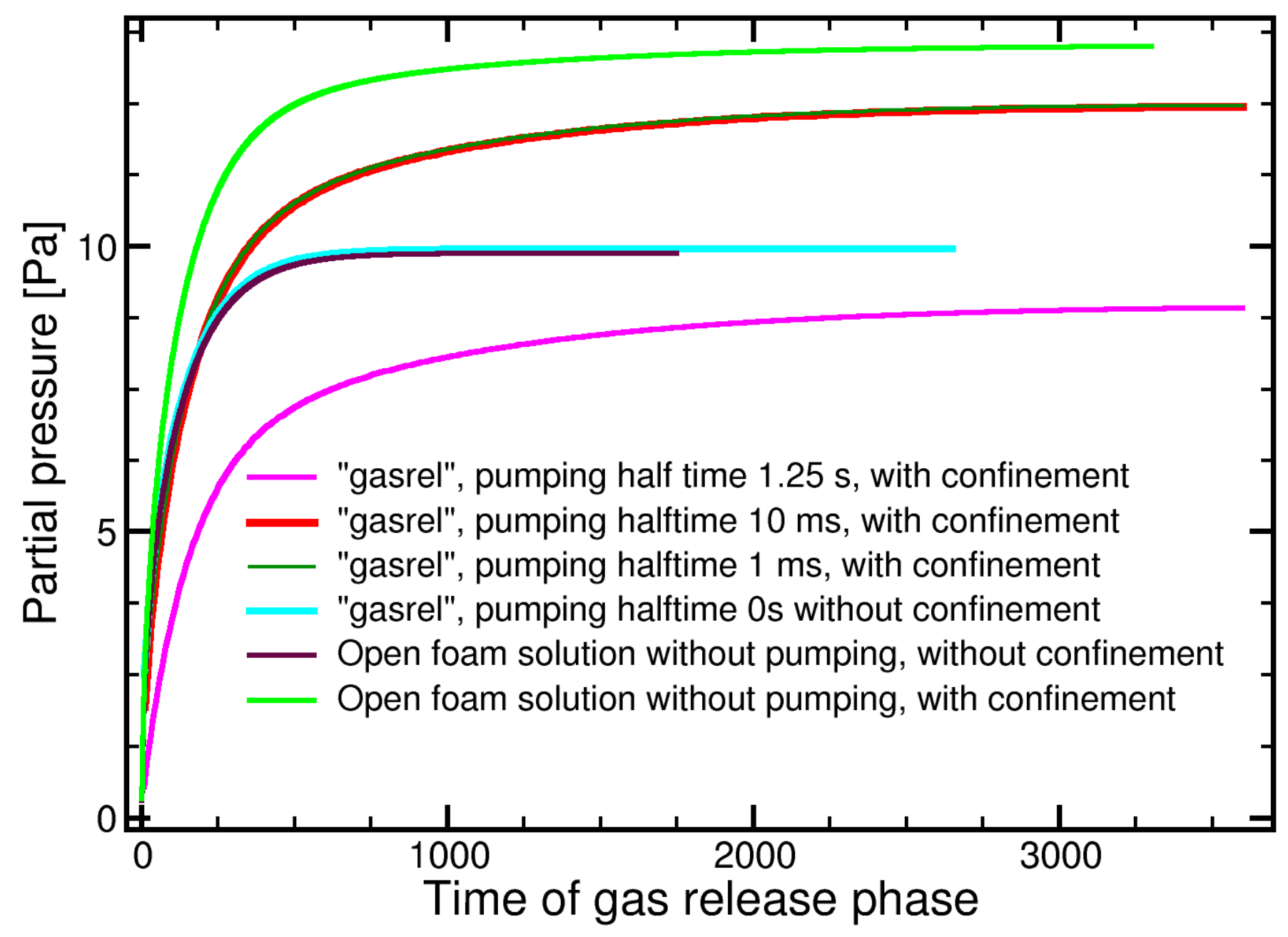


$\mathrm{T}=773 \mathrm{~K}, \mathrm{Cu}$ containment with Optifer specimen

chamber dimension $80 \mathrm{~mm}$ to $40 \mathrm{~mm}$ diameter, $p_{\text {load }}=310^{3} \mathrm{~Pa}$, interstitial-molecular

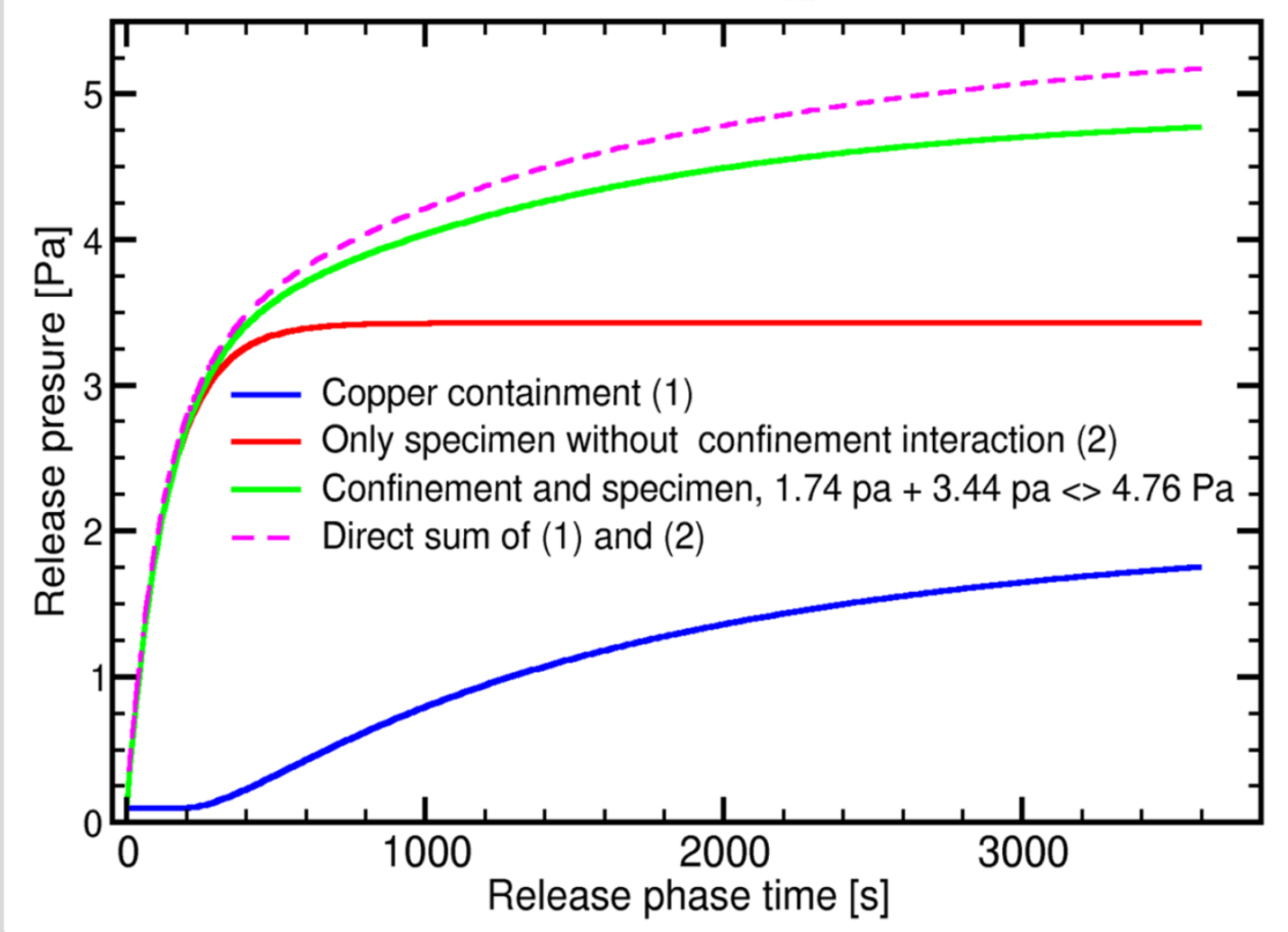

$\mathbb{N}$ CIT

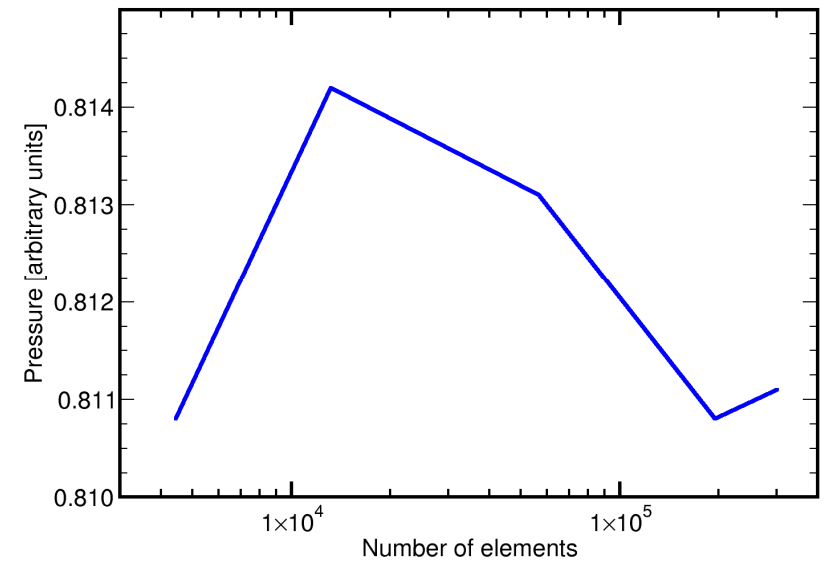

Choosing starting pressure:

Weak pump, half time $10 \mathrm{~s}$, total pressure $10^{5} \mathrm{Pas}, 3 \% \mathrm{D}_{2}$

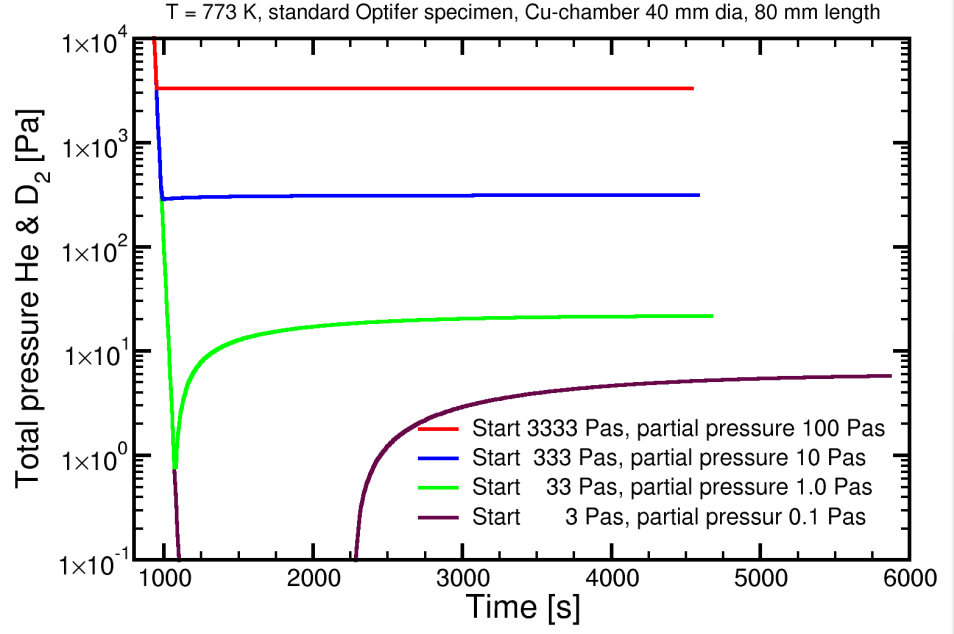




\section{Results for conception of experiments}

$20 \mathrm{~mm}$ diameter, pumping halftime $0.5 \mathrm{~s}, 773 \mathrm{~K}$ Total start pressure $33 \mathrm{Pas}$

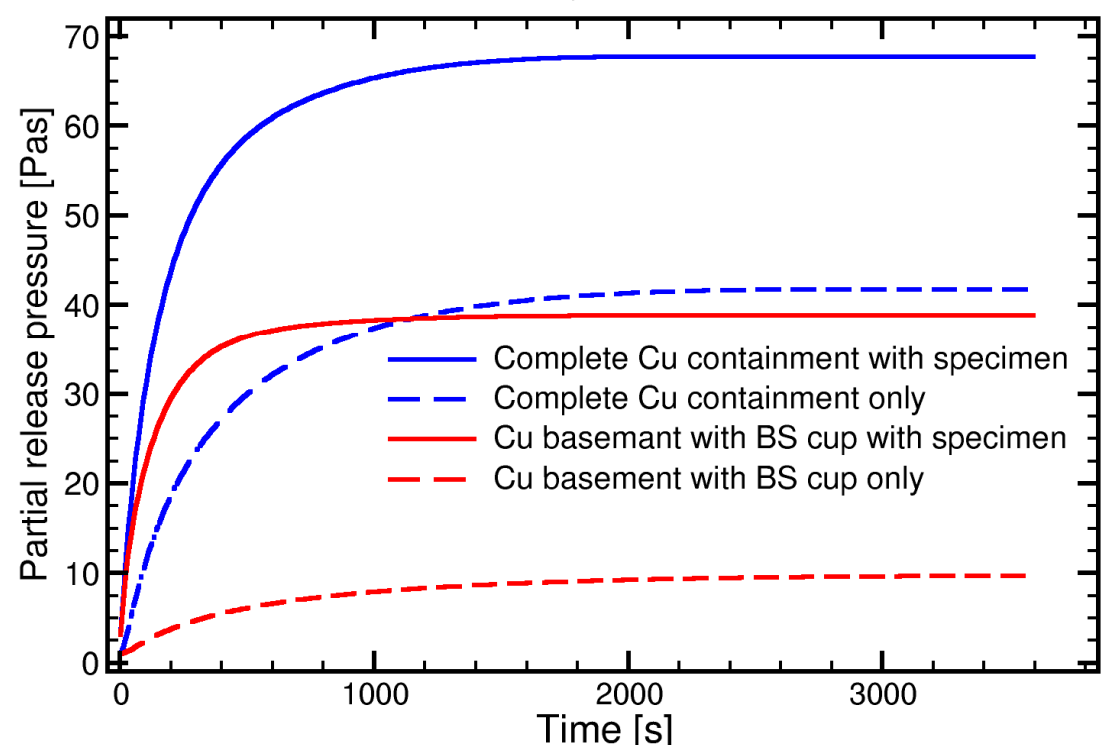

$40 \mathrm{~mm}$ diameter, pumping halftime $0.5 \mathrm{~s}, 773 \mathrm{~K}$

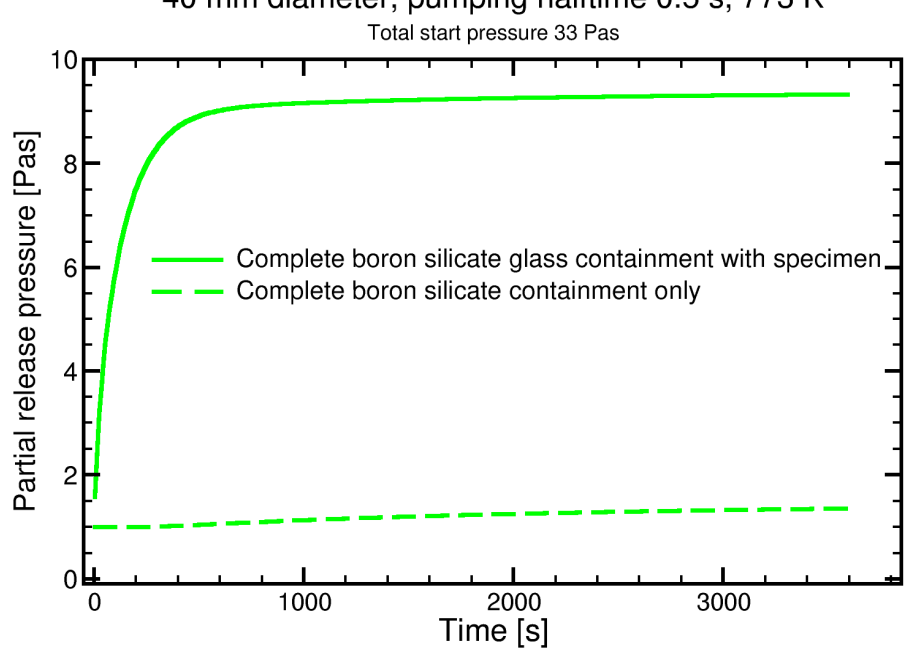

Copper or Boron Silicate glass

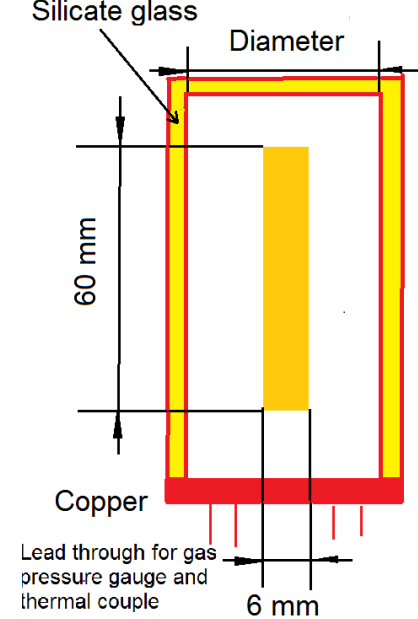

$40 \mathrm{~mm}$ diameter, pumping halftime $0.5 \mathrm{~s}, 773 \mathrm{~K}$ Total start pressure $33 \mathrm{Pas}$

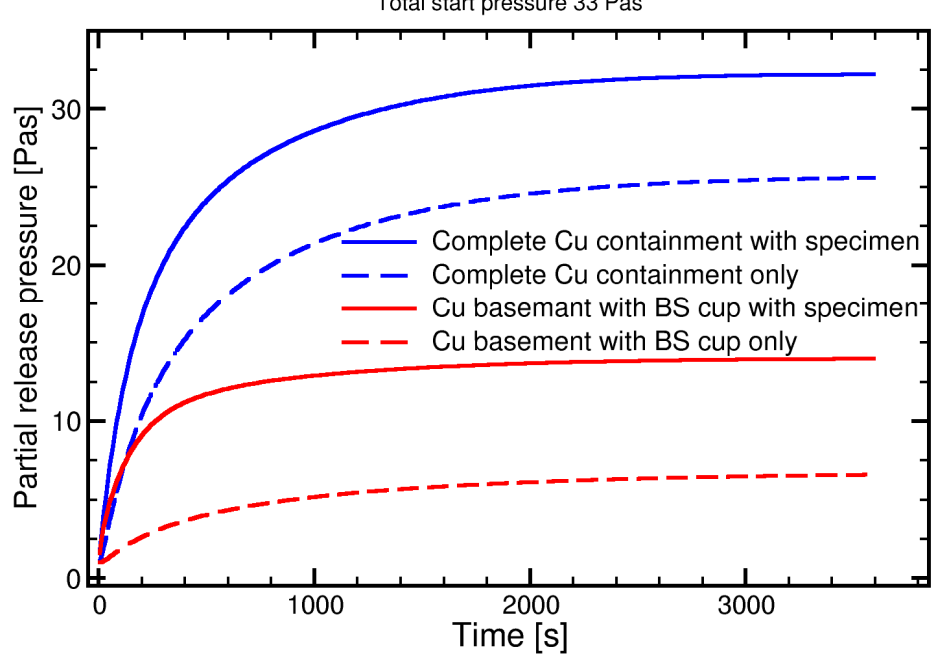

$80 \mathrm{~mm}$ diameter, pumping halftime $0.5 \mathrm{~s}, 773 \mathrm{~K}$

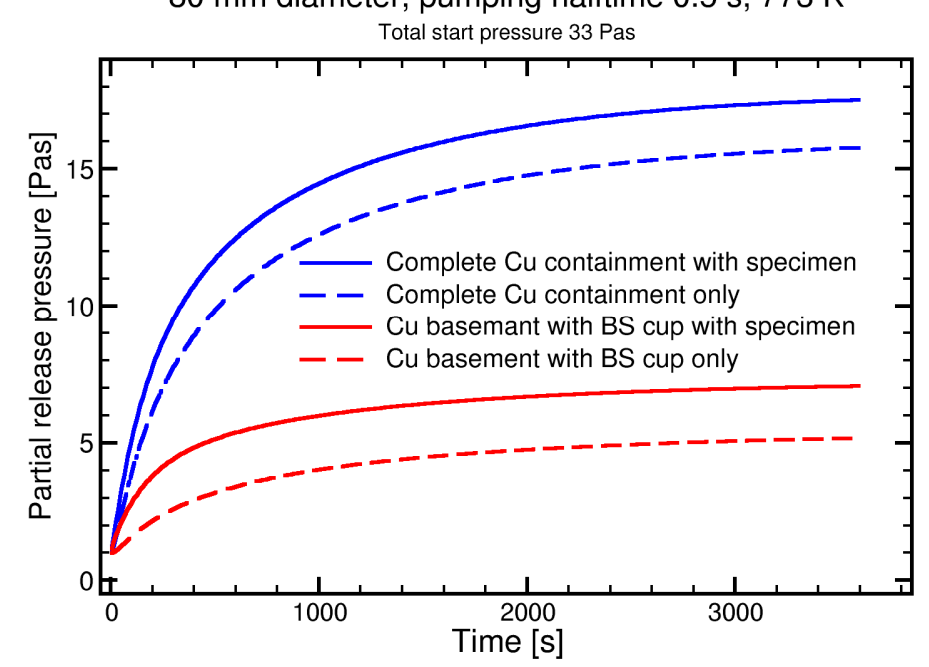




\section{5.: Outlook to analytical solution}

Solution in the charging interval

$c(r, z, t)=k_{s, s a} \sqrt{p_{\text {load }}} \sum_{n, m} \frac{8(-1)^{n+1}}{\pi(2 n+1) x_{m} J_{1}\left(x_{m}\right)} \exp \left(-\gamma_{n, m}^{2} t\right) \cos \left((2 n+1) \frac{\pi}{2} z\right) J_{0}\left(x_{m} r\right)$

where

$\gamma_{n, m}^{2}=D_{s a}\left(\frac{x_{m}^{2}}{r_{s}^{2}}+\frac{(2 n+1)^{2} \pi^{2}}{4 z_{s}^{2}}\right), J_{\alpha}(x)(\alpha=0,1)$ Bessel functions of the first kind,

$x_{m}$ the $m$-th roots of $J_{0}(x)$.
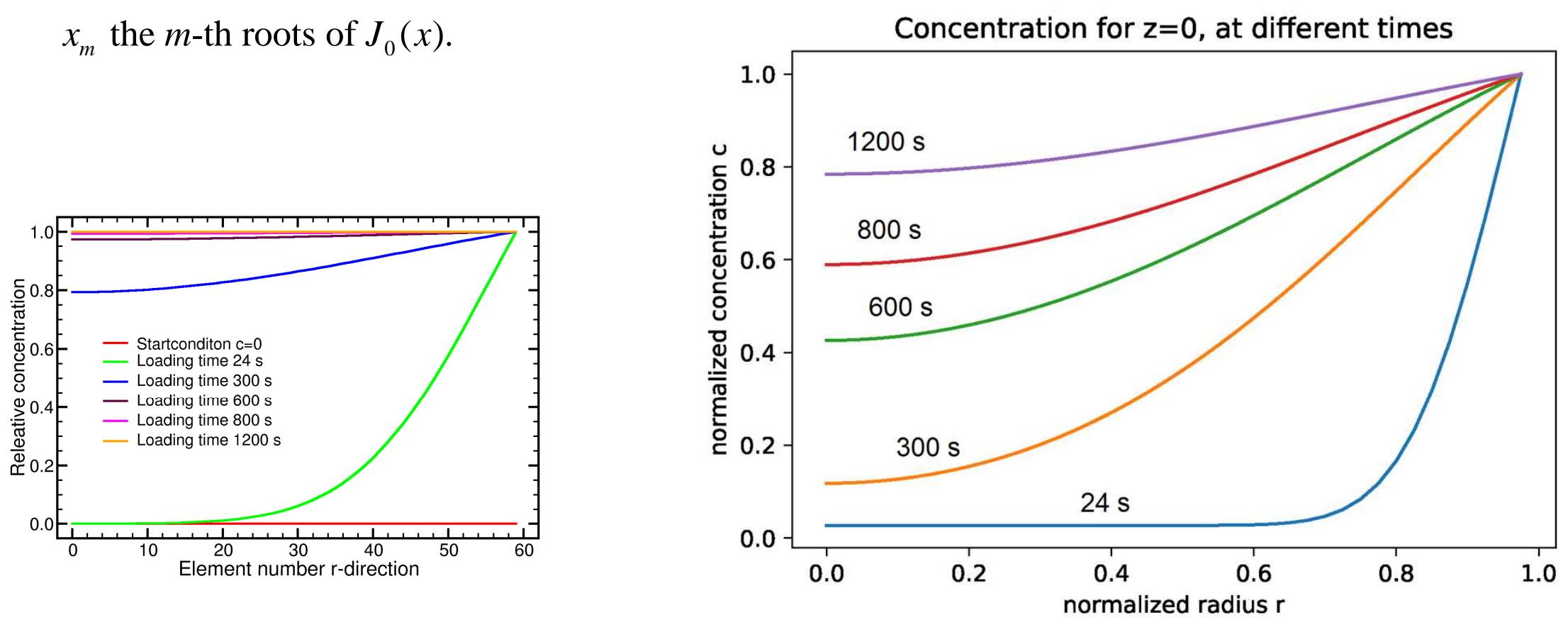


\title{
NUMERICAL ANALYSIS OF AN ISOVOLUMETRIC THERMAL DESORPTION EXPERIMENT
}

\author{
A. von der Weth, K. Nagatou,
}

F. Arbeiter, R. Dagan, D. Klimenko, V. Pasler, M. Schulz, INR, KIT, CN, Athens, 25th June, 2019

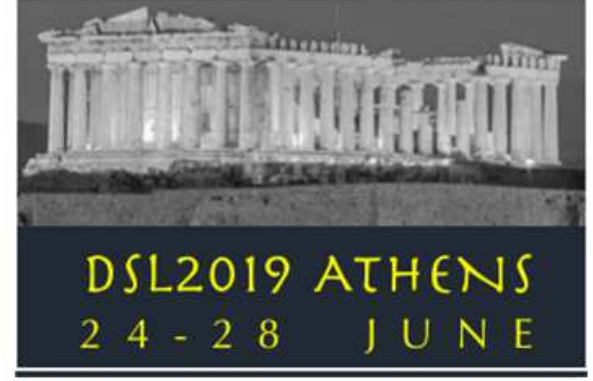

1.: Description of setup

2.: Simple analytical solutions

3.: Structure of Differential Equations

4.: Results of numerical optimization

5.: Outlook to analytical solution 


\section{Reminder: gas release diffusion experiments}

\section{Procedure:}

1. Specimen placed in defined volume enclusure

2. Load specimen with hydrogen at defined conditions $\left(T, \mathrm{p}_{\mathrm{H} 2}\right)$

3. Swiftly evacuate gases from enclosure

4. Measure pressure increase $p(t)$ in enclosure

$\rightarrow$ Sieverts' constant can be deduced from final pressure

$\rightarrow$ Diffusion constant can be deduced from pressure rise phase
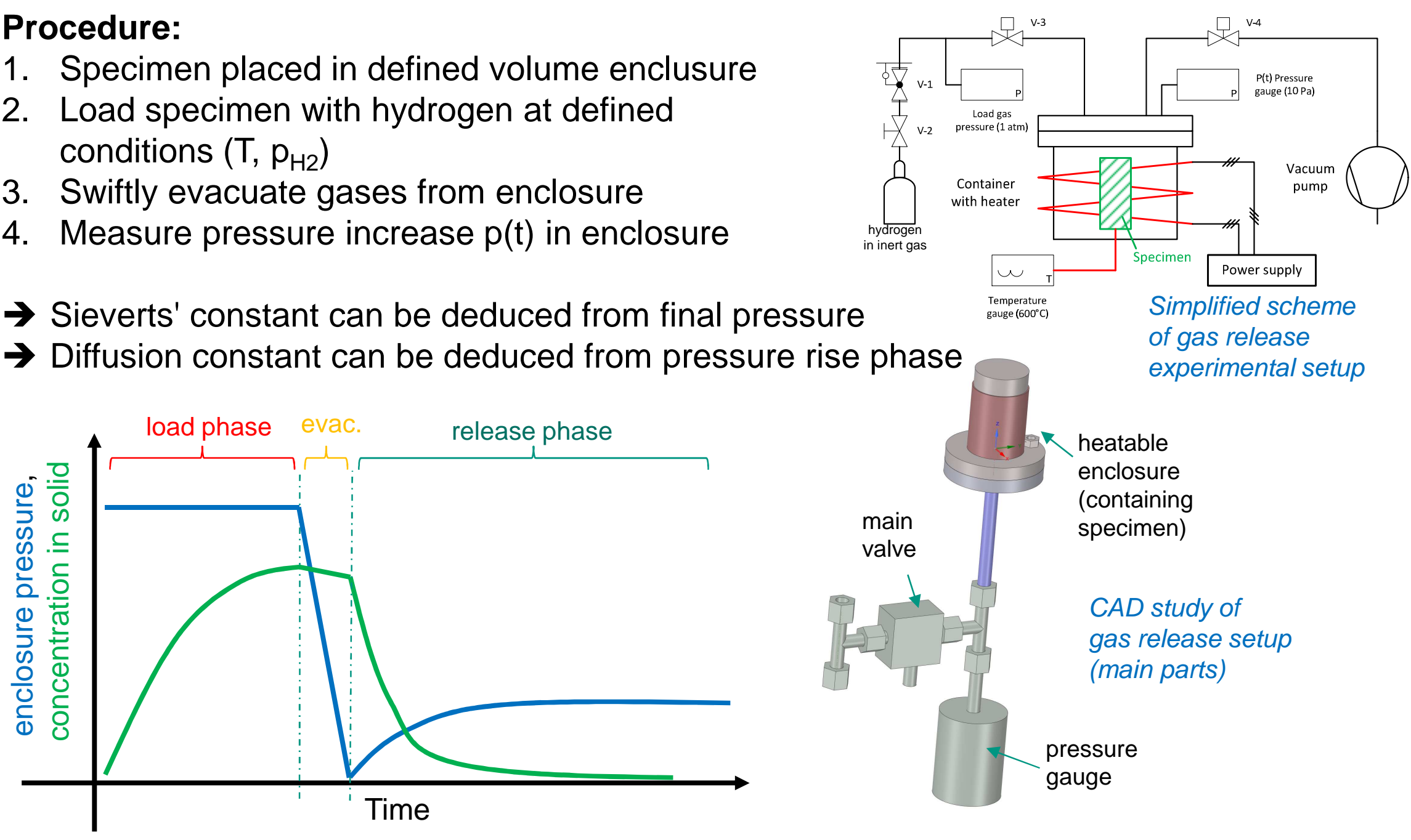


\section{1.: Description of setup of an Isovoluminetric Thermal}

Desorption Experiment

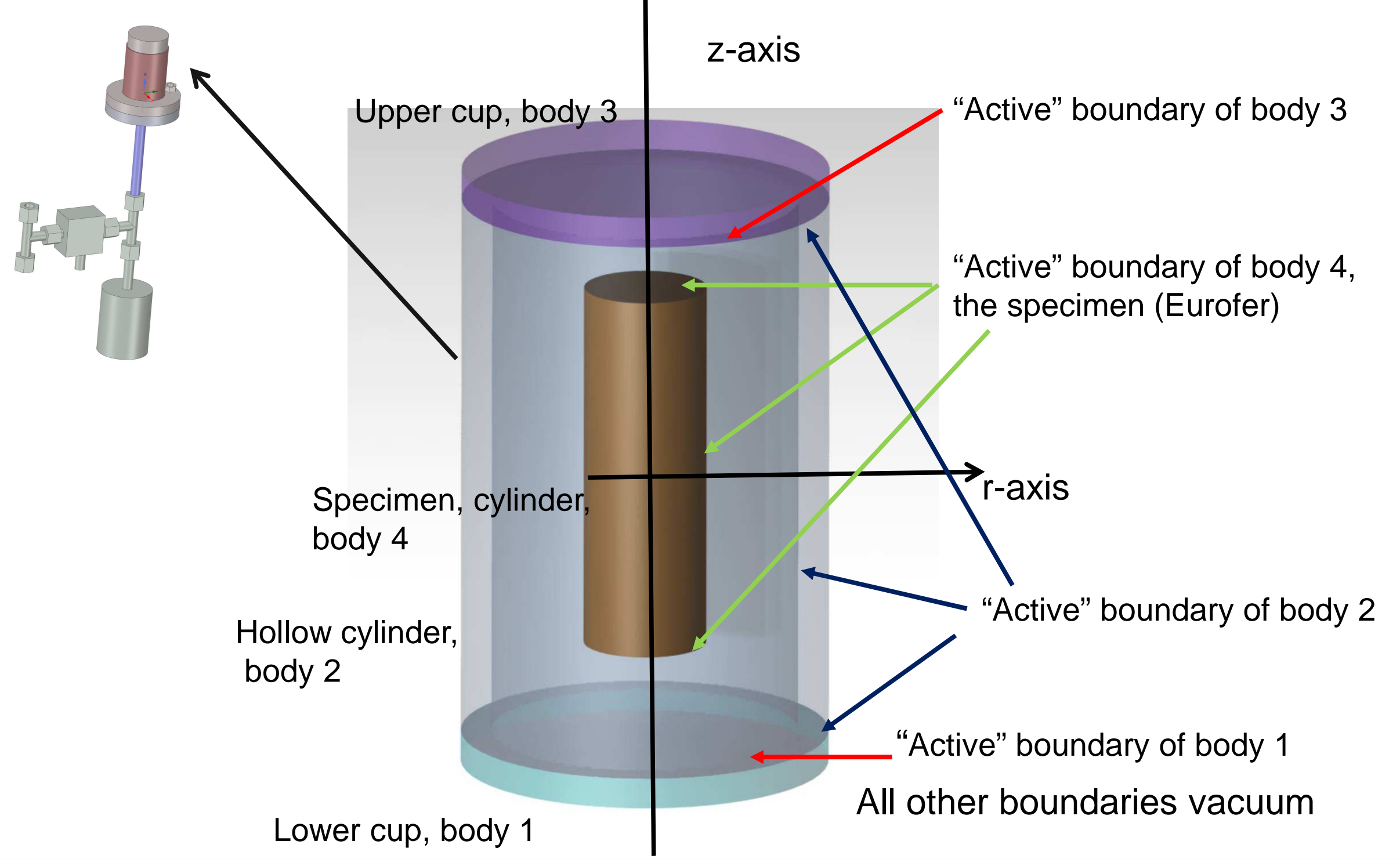




\section{1.: Physical description}

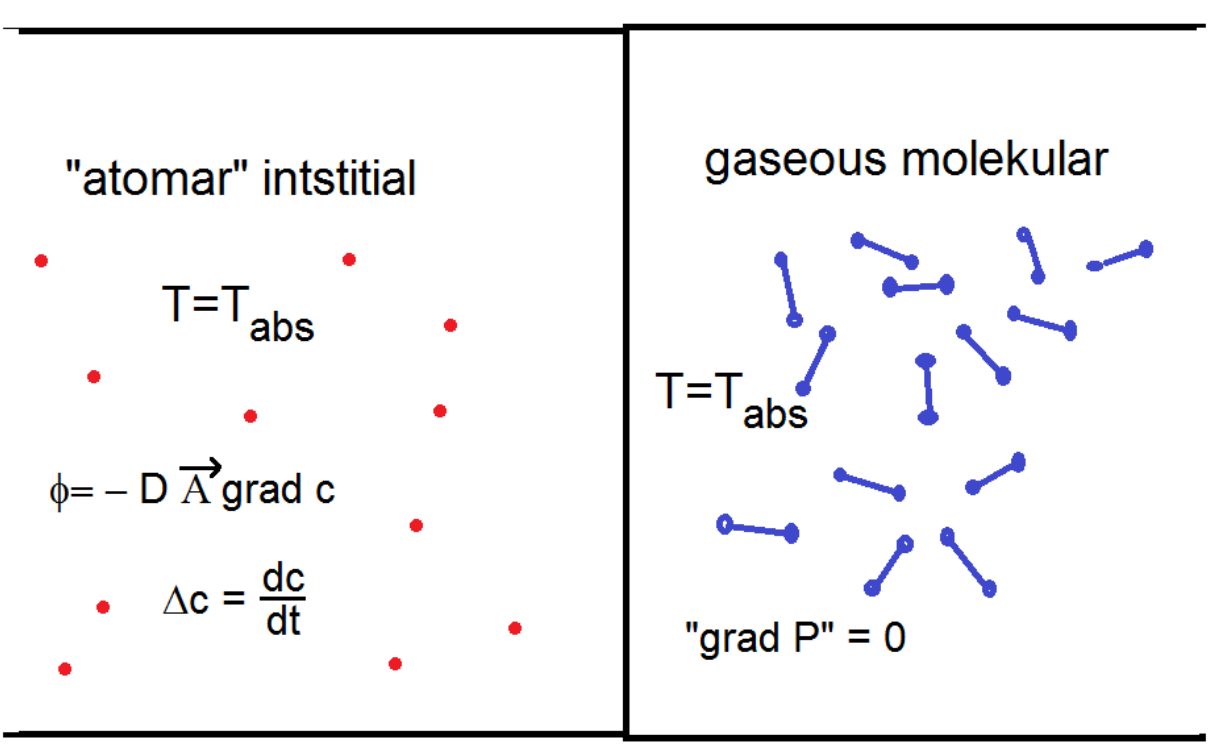

"Velocity" of mass transfer by diffusion constant D

Ratio of densities given by Sieverts' law (phase equilibrium)

$$
c=k_{s} \sqrt{p_{r}}
$$

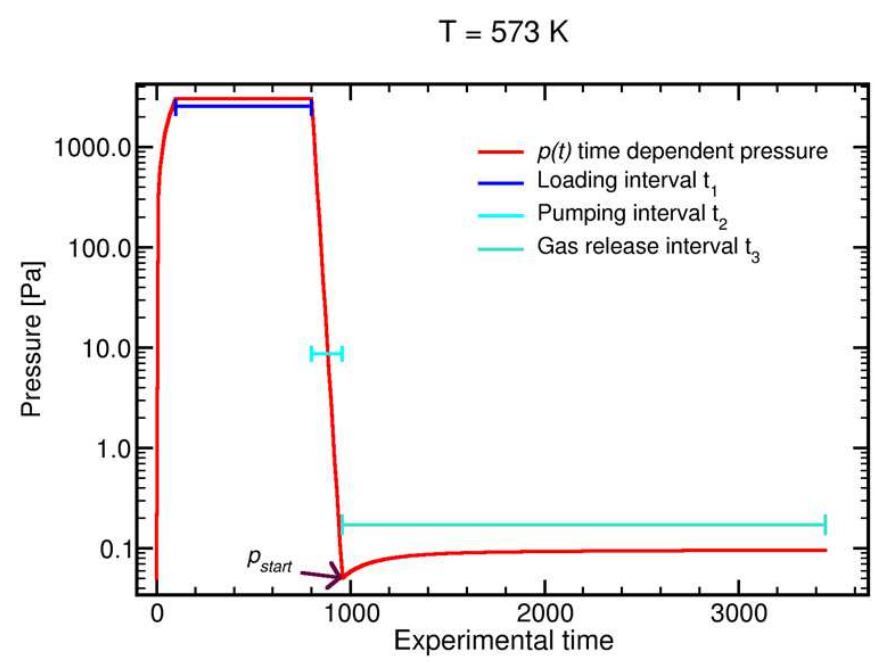

Removable cup

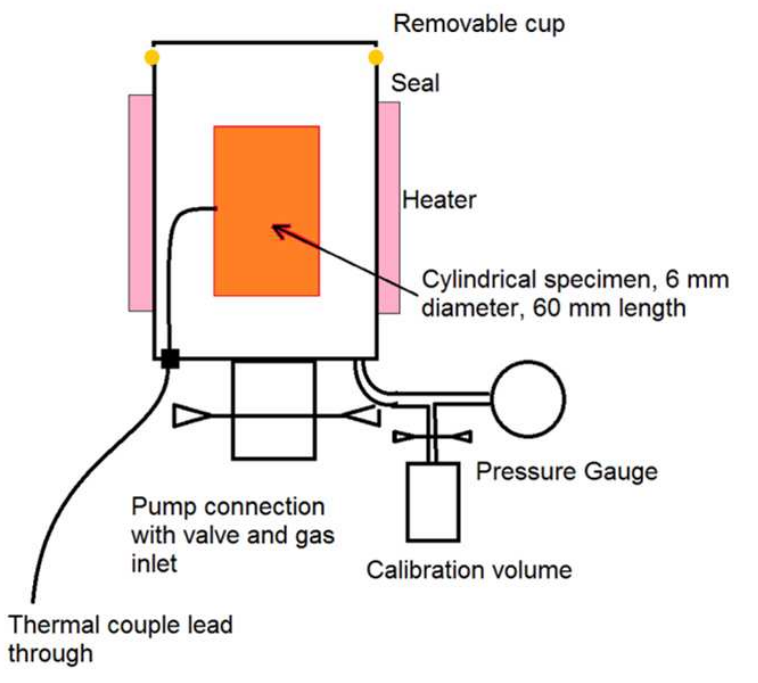




\section{2.: Simple analytical Solutions}

Without re-diffusion, complete outgassing, $c_{\text {sample }}=0$ :

$$
\underbrace{p_{r}}_{t \rightarrow \infty}=\underbrace{p_{\text {end }}}_{\begin{array}{c}
\text { switching off } \\
\text { pump }
\end{array}}+\frac{V_{s a} k_{s, s a} \sqrt{p_{\text {load }}} R T}{\begin{array}{c}
V_{c}-V_{\text {chamer }}-V_{\text {sample }} \\
\text { sample }
\end{array}}
$$

With phase equilibrium, mass conservation (number of "hydrogens" in atomic interstitial and molecular gaseous state constant) and non interacting confinement condition, currently unused:

$$
0=\frac{2\left(V_{c}-V_{s a}\right)}{R T}\left(\sqrt{p_{r}}\right)^{2}+V_{s} k_{s, s a} \underbrace{\sqrt{p_{r}}}_{=" x^{\prime \prime}}-\left(V_{s} k_{s, s a} \sqrt{p_{\text {load }}}+\frac{2 p_{\text {end }}\left(V_{c}-V_{s a}\right)}{R T}\right)
$$

$$
k_{s, s a}=\frac{2\left(V_{c}-V_{s a}\right)}{R T V_{s a}} \frac{\left(p_{r}-p_{\text {end }}\right)}{\sqrt{p_{\text {load }}-\sqrt{p_{r}}}}
$$

Experimentally difficult realization of boundaries, no statement about diffusion constant

$$
\underbrace{p_{r}}_{t \rightarrow \infty}=\left(\frac{-1 \underbrace{+}_{n e g . s q r t} \sqrt{1+\left(\frac{8\left(V_{c}-V_{s a}\right) \sqrt{p_{\text {load }}}}{R T k_{s, s a}}+\frac{16 p_{\text {end }}\left(V_{c}-V_{s a}\right)^{2}}{\left(R T V_{s a} k_{s, s a}\right)^{2}}\right)}}{\left(\frac{V_{s a} k_{s, s a} R T}{4\left(V_{c}-V_{s a}\right)}\right)^{-1}}\right)^{2}
$$




\section{3.: Structure of differential equations:}

$$
\begin{aligned}
& \frac{\partial c}{\partial t}=D_{s a} \Delta c \quad \frac{\partial d(i)}{\partial t}=D_{c u} \Delta d(i), i=1,2,3 \\
& \Delta=\frac{\partial^{2}}{\partial r^{2}}+\frac{1}{r} \frac{\partial}{\partial r}+\frac{\partial^{2}}{\partial z^{2}}+\underbrace{\frac{1}{r^{2}} \frac{\partial^{2}}{\partial \varphi^{2}}}_{=0} \\
& c\left(0 \leq r \leq r_{s}, z= \pm z_{s}, \forall t\right)=k_{s, s a} \sqrt{p(t)} \\
& c\left(r=r_{s},|z| \leq z_{s}, \forall t\right)=k_{s, s a} \sqrt{p(t)} \\
& d(1)\left(r \leq r_{c o}, z=-z_{c i}, \forall t\right)=k_{s, c u} \sqrt{p(t)} \\
& d(2)\left(r=r_{c i},-z_{c i} \leq z \leq z_{c i}, \forall t\right)=k_{s, c u} \sqrt{p(t)} \\
& d(2)\left(r_{c i} \leq r \leq r_{c o}, z= \pm z_{c i}, \forall t\right)=k_{s, c u} \sqrt{p(t)} \\
& d(3)\left(r \leq r_{c o}, z=z_{c i}, \forall t\right)=k_{s, c u} \sqrt{p(t)}
\end{aligned}
$$

Analytical 1d: Sedano, Perujo (1999), Esteban Douglas (2001) , Eichenauer Pebler (1957) Hattenbach (1961)

Analytical 2d: Eichenauer, Pebler, Witte 1965

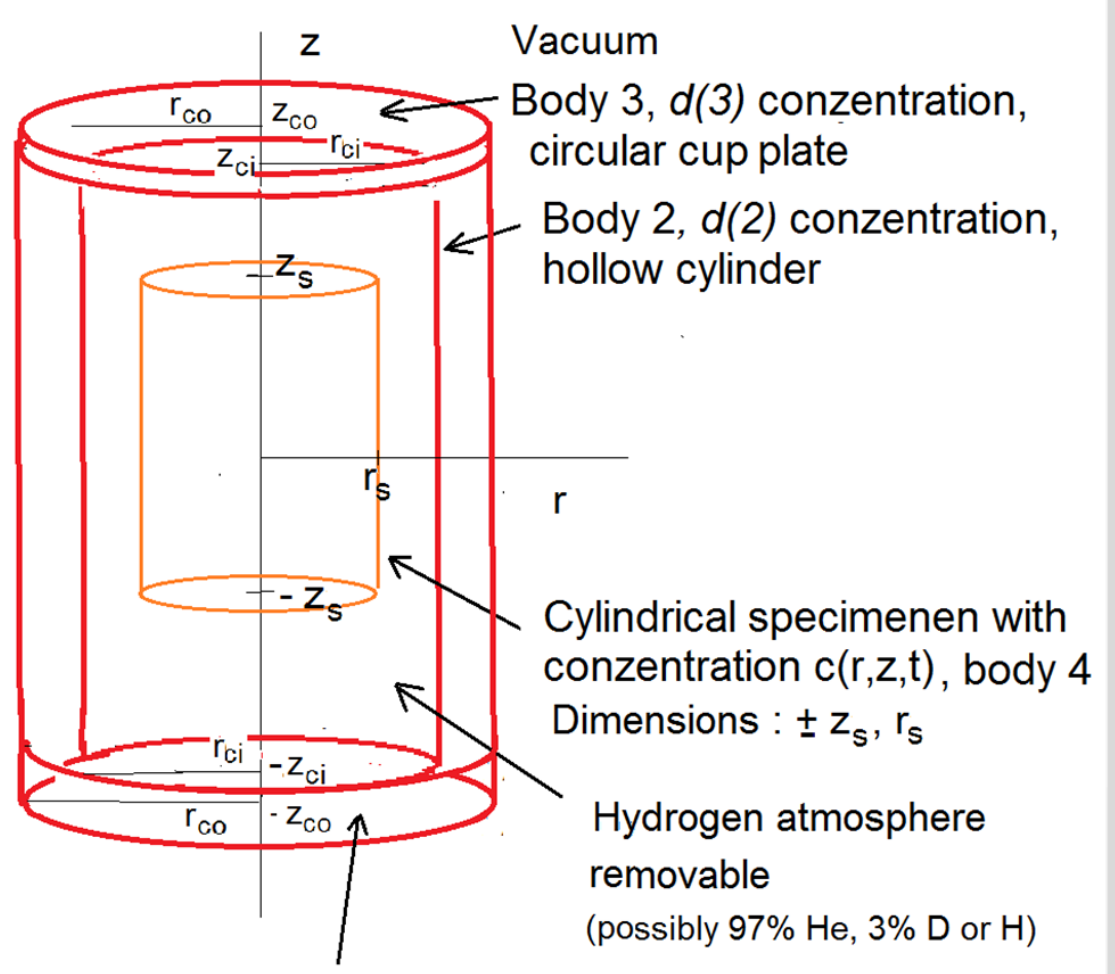

Body 1, $d(1)$ conzentration 


\section{Structure of differential equations}

$$
\frac{d m}{d t}=-2 \pi D_{s a} \int_{0}^{r_{s}} \underbrace{2}_{\text {symmetric }} r \frac{\partial}{\partial z} c\left(r, z=z_{s}, t\right) d r
$$

- $\underbrace{2 \pi r_{s} D_{s a} \int_{-z_{s}}^{z_{s}} \frac{\partial}{\partial r} c\left(r=r_{s}, z, t\right) d z}_{\text {superficies surface }}$

superficies surface of specimen

- $\underbrace{2 \pi D_{c u} \int_{0}^{r_{c o}} r \frac{\partial}{\partial z} d(1)\left(r, z=-z_{c i}, t\right) d r}$ circular aerea of body 1

- $2 \pi r_{c i} D_{c u} \int_{-z_{c i}}^{z_{c i}} \frac{\partial}{\partial r} d(2)\left(r=r_{c i}, z, t\right) d z$ superficies surface of body 2 $-\underbrace{2 \pi D_{c u} \int_{0}^{r_{c o}} r \frac{\partial}{\partial z} d(3)\left(r, z=z_{c i}, t\right) d r}_{\text {circular area of body } 3}$

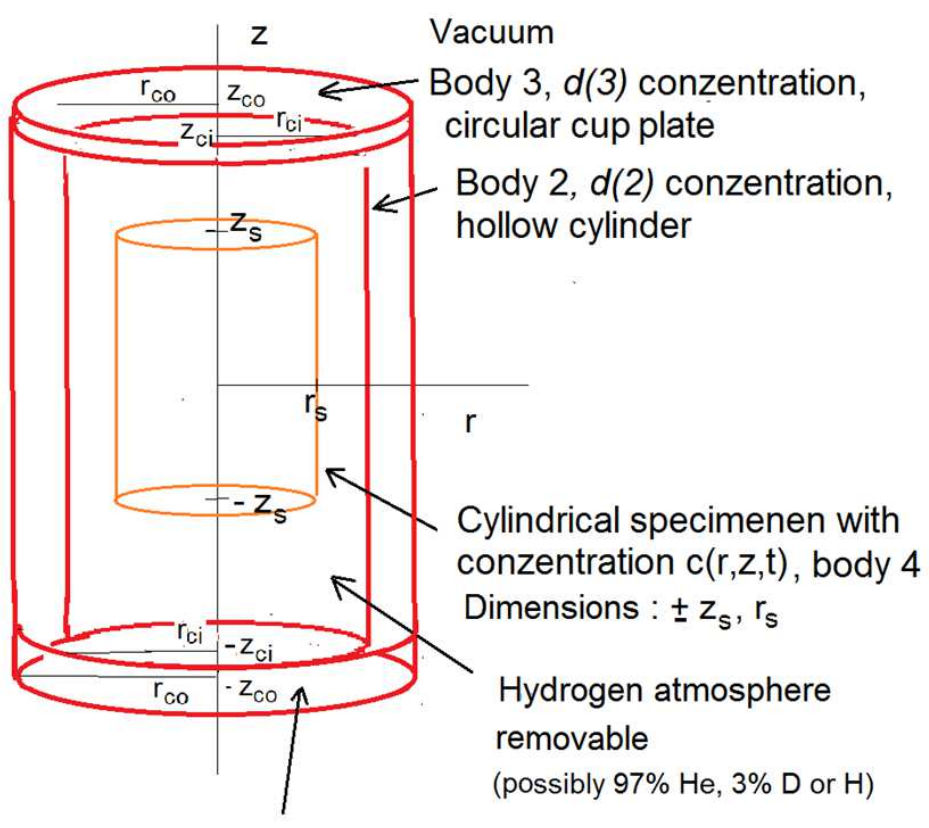

Body 1, d(1) conzentration

$$
p(t)=p_{\text {start }}+\underbrace{k_{v}}_{R T_{\text {abs }} / V_{\text {gas }}} \int_{t_{1}+t_{2}}^{t} \underbrace{0.5}_{\begin{array}{c}
\text { gaseous } \\
\text { interstitial }
\end{array}} \frac{d m}{d t} d t
$$




\section{4.: Results of numerical solution}

$\mathrm{T}=773 \mathrm{~K}, \mathrm{Cu}$ containment with Optifer specimen, 13. 5. chamber dimension $80 \mathrm{~mm}$ to $40 \mathrm{~mm}$ diameter, $\mathrm{p}_{\text {load }}=310^{3} \mathrm{~Pa}$, improved gradient calculation

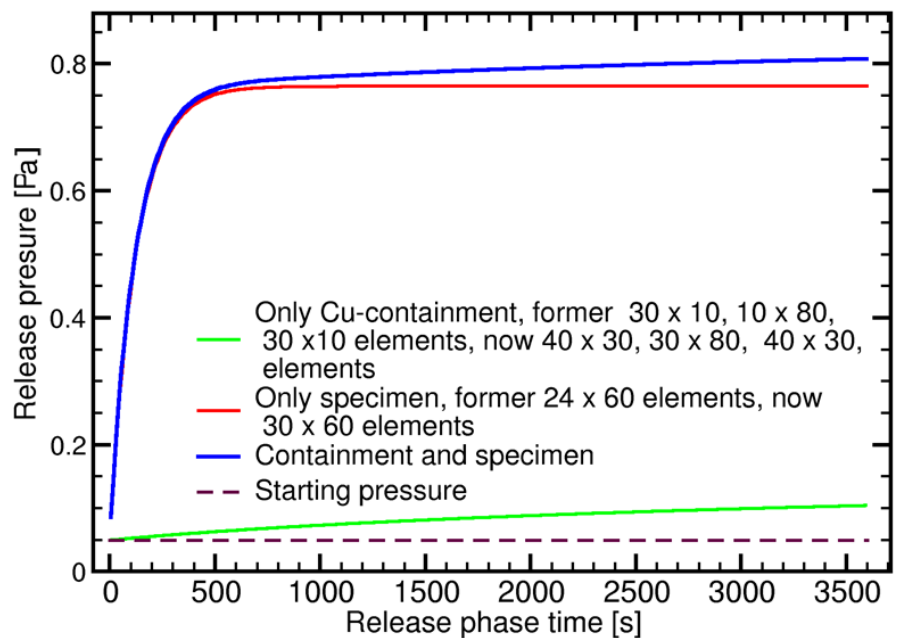

Simple numerical gradient calculation

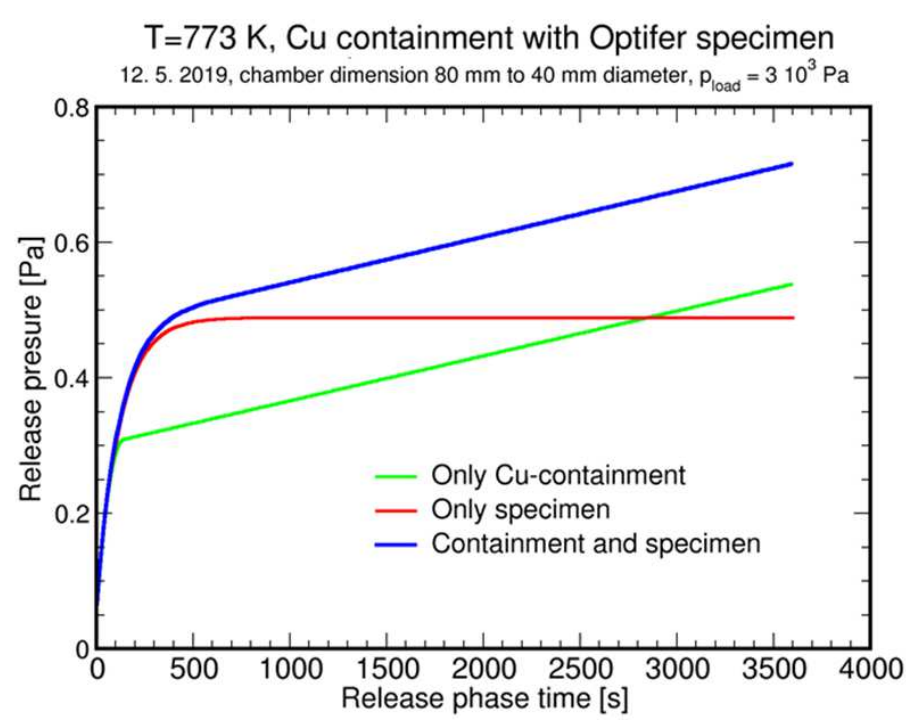

\section{Numerical artefacts}

Development, $\mathrm{T}=773 \mathrm{~K}$, Cu cont. with Optifer specimen chamber dimension $80 \mathrm{~mm}$ to $40 \mathrm{~mm}$ diameter, $\mathrm{p}_{\text {load }}=310^{3} \mathrm{~Pa}$, improved gradient calculation

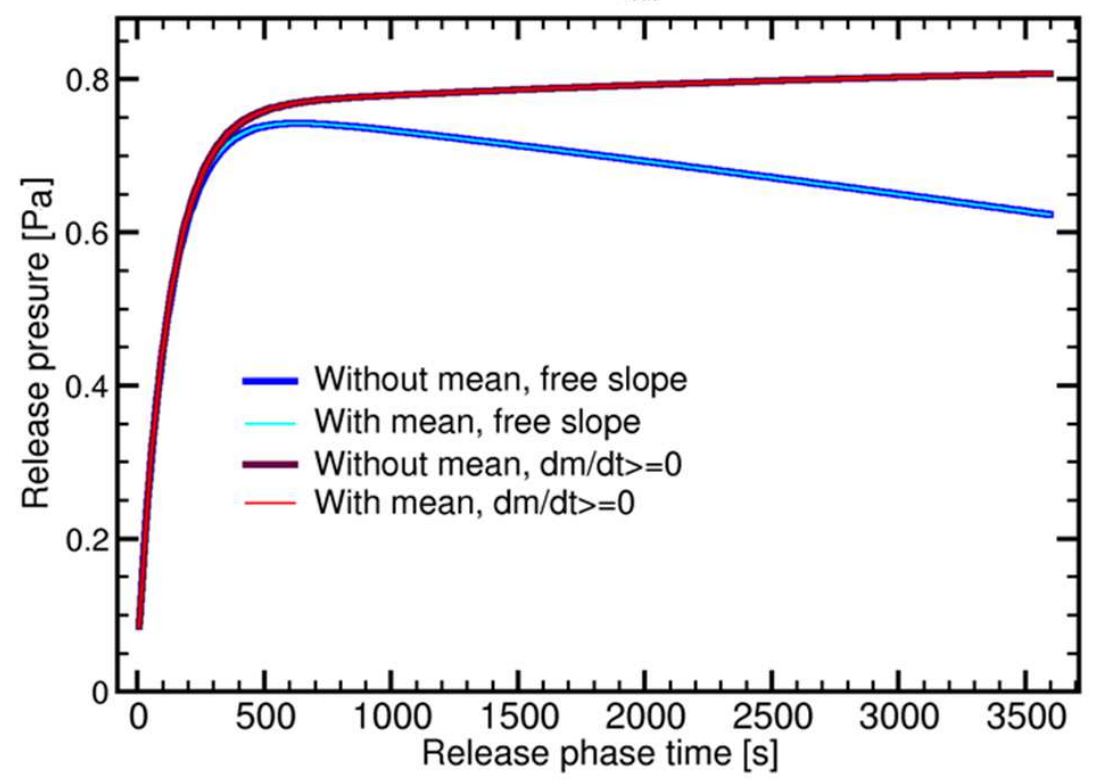




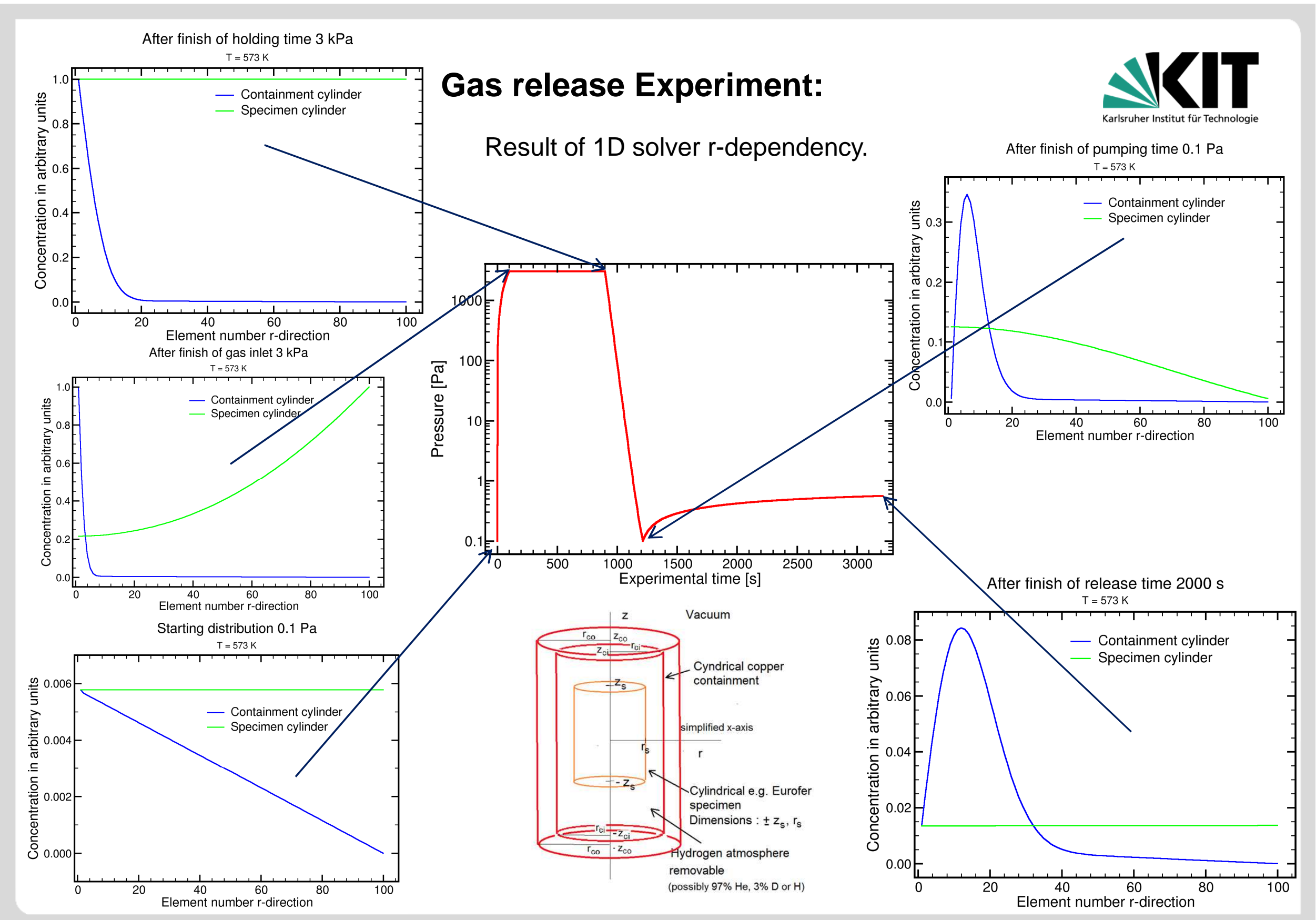

$925^{\text {th }}$ of June, NUMERICAL ANALYSIS OF AN ISOVOLUMETRIC THERMAL DESORPTION EXPERIMENT

MET/INR/Maschinenbau 


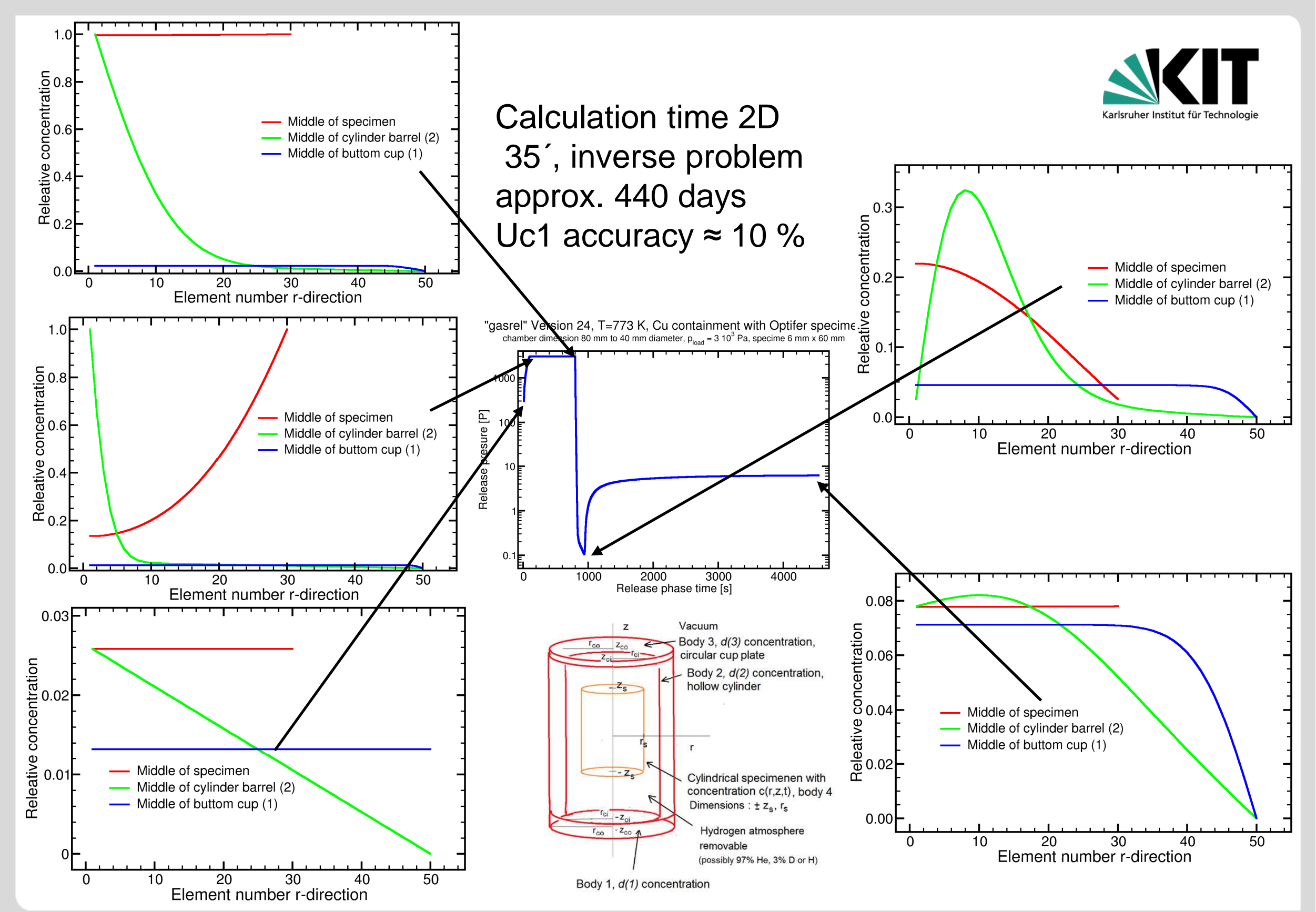




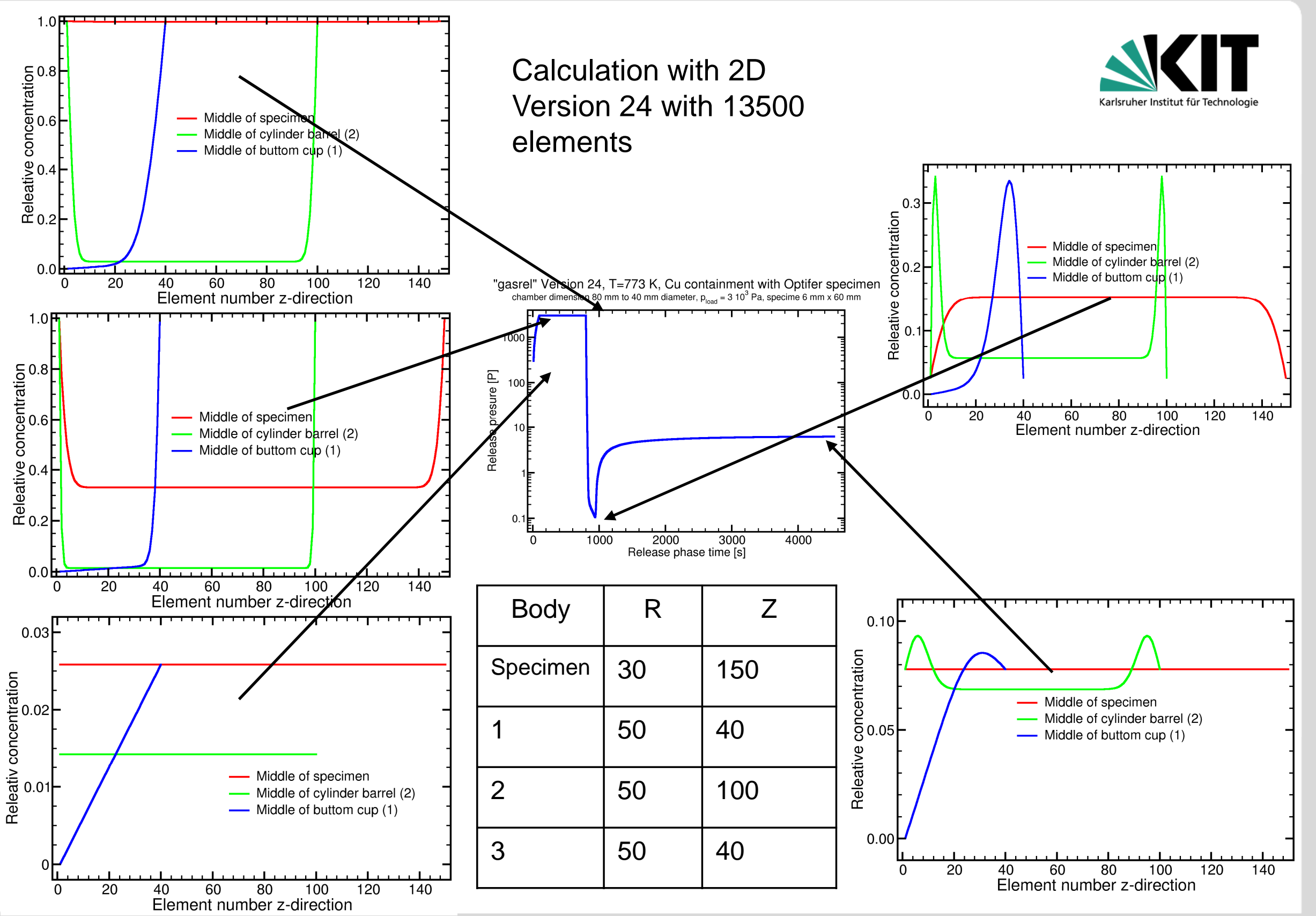

$1125^{\text {th }}$ of June, NUMERICAL ANALYSIS OF AN ISOVOLUMETRIC THERMAL DESORPTION EXPERIMENT

MET/INR/Maschinenbau 


\section{Comparison with analytical solution:}

Results for chamber $40 \mathrm{~mm}$ dia. and $80 \mathrm{~mm}$ length, Optifer

$\mathrm{p}_{\text {load }}=310^{3} \mathrm{~Pa}$, integration time checked $1 \mathrm{~ms}, 0.5 \mathrm{~ms}, 0.1 \mathrm{~ms}$ less than $1 / 1000$ deviation

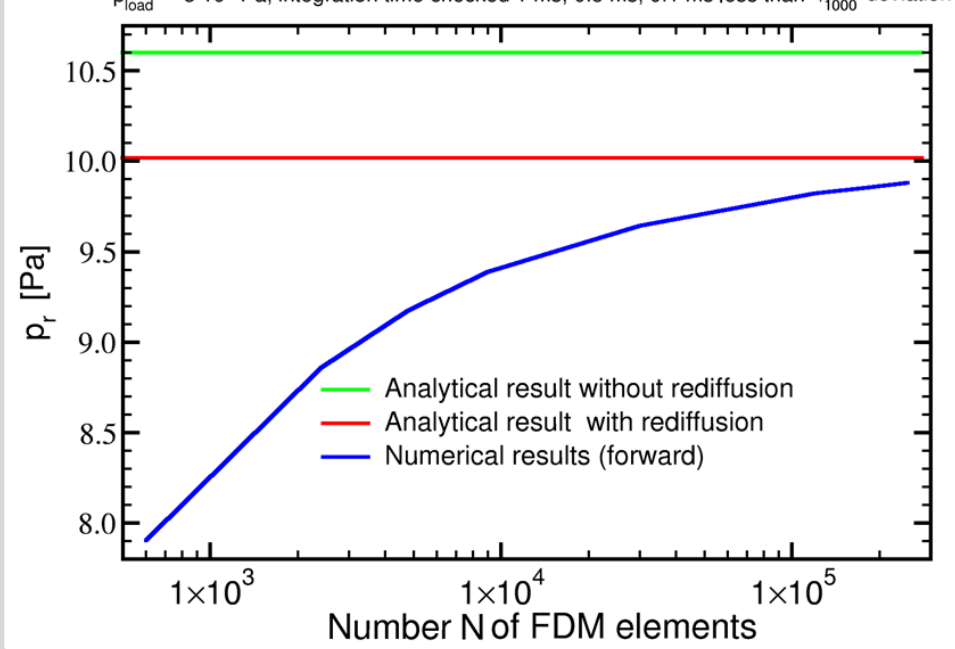

$10^{-11}<\frac{D_{s a} \Delta t}{\Delta z^{2}}<10^{-6}$

Results for chamber 40/20 mm dia. and $80 \mathrm{~mm}$ length, Optifer cylinder $60 \mathrm{~mm} \times 6 \mathrm{~mm}$ $\mathrm{p}_{\text {load }}=310^{3} \mathrm{~Pa}$, integration time $1 \mathrm{~ms}, 0.5 \mathrm{~ms}, 0.1 \mathrm{~ms}$ less than ${ }_{1}^{1}{ }_{1000}$ deviation

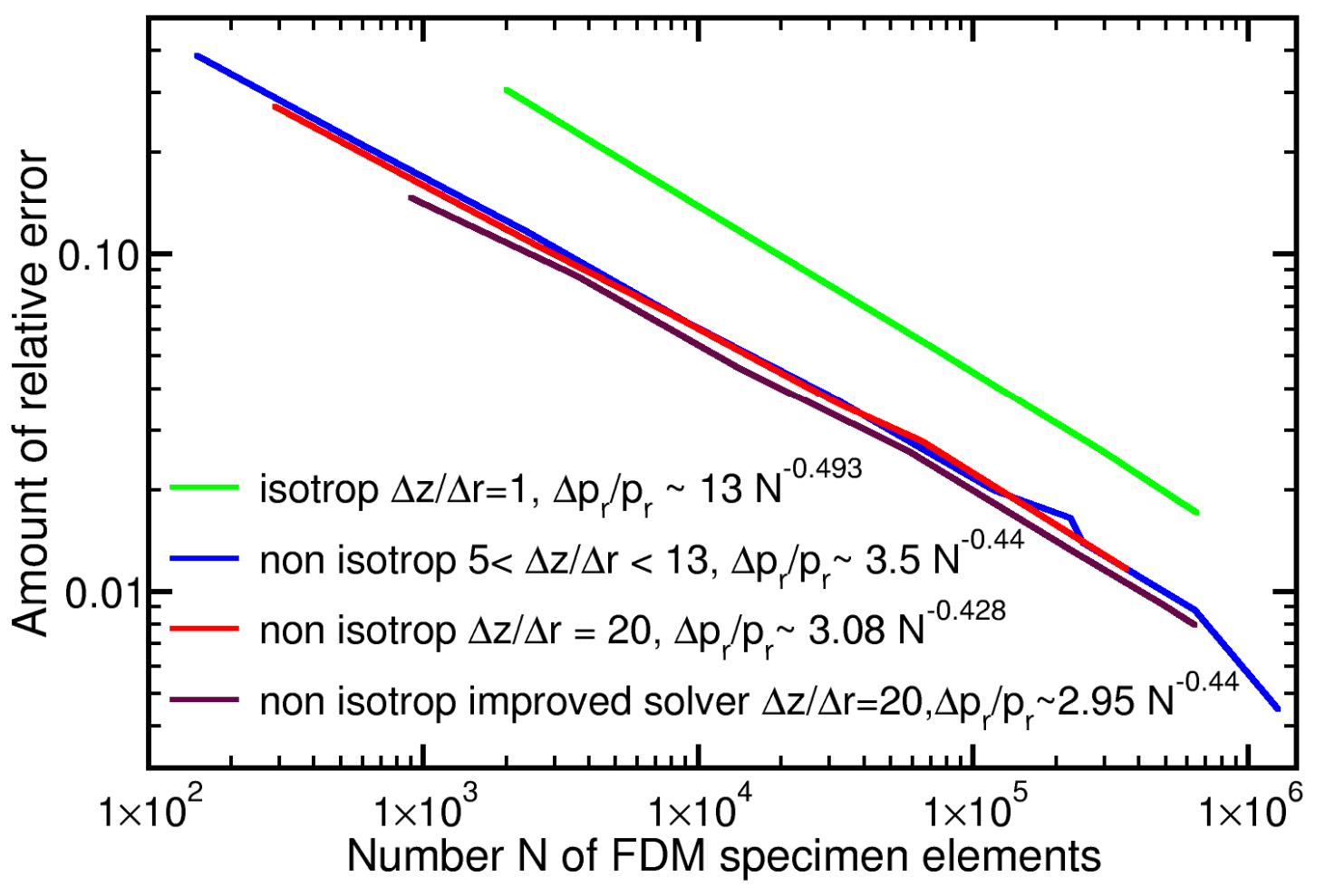


Small excurse to solver algorithms:

1D forward Euler

$$
\begin{gathered}
c(i, t+\Delta t)=c(i, t)+\frac{D \Delta t}{\Delta r^{2}}(c(i+1, t)+c(i-1, t)-2 c(i, t))+ \\
\frac{D \Delta t}{2 r \Delta r}((c(i+1, t))-c(i-1, t))
\end{gathered}
$$

2D improved forward Euler:

$$
\begin{aligned}
\mathrm{c}(i, j, t+\Delta t) & \\
=c(i, j, t) & +\frac{D \Delta t}{2 i \Delta r^{2}}((2 i+1) c(i+1, j, t)+(2 i-1) c(i-1, j, t)-(4 i) c(i, t)) \\
& +\frac{D \Delta t}{\Delta z^{2}}((c(i, j+1, t))+c(i, j-1, t)-2 c(i, j))
\end{aligned}
$$

Desired: Backward Euler solver, e. g. 1D cartesian:

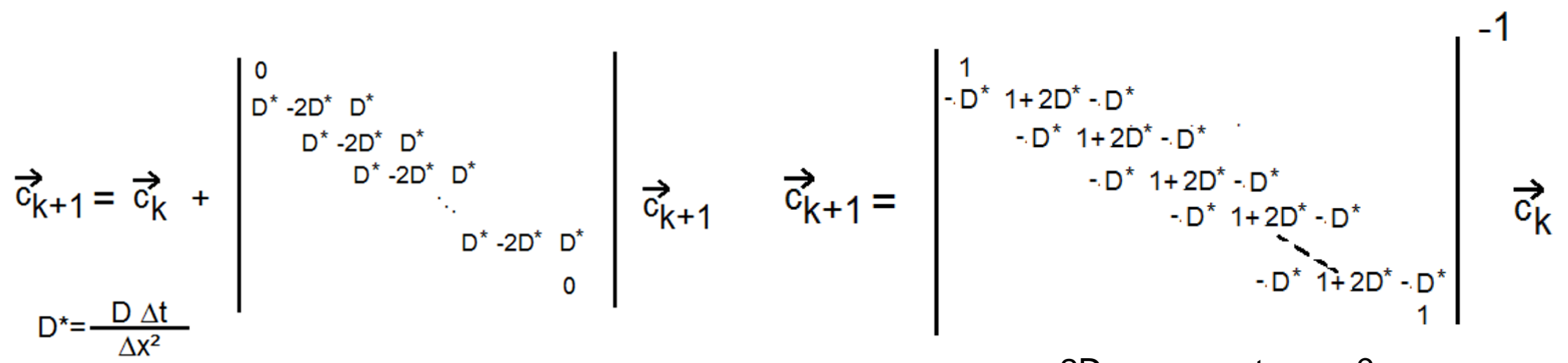

$2 \mathrm{D} \mathrm{n} \times \mathrm{n} \times \mathrm{m}$ tensor? 


\section{4.: Results}

"gasrel" calculation with 6400 specimen element and 1800 conf. elements 14 halftimes reaching endpresure $(0.1 \mathrm{~Pa})$

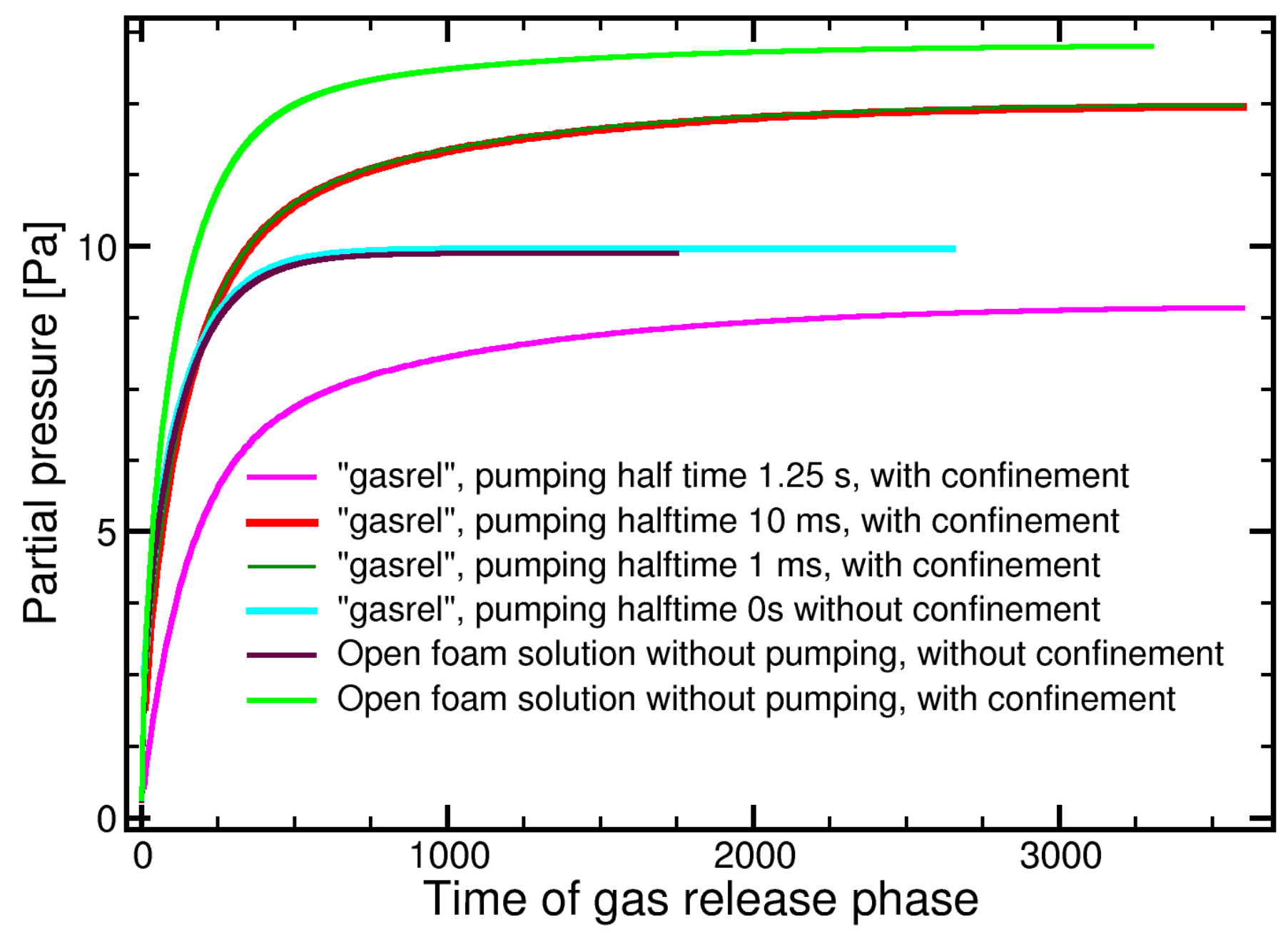


$\mathrm{T}=773 \mathrm{~K}, \mathrm{Cu}$ containment with Optifer specimen

chamber dimension $80 \mathrm{~mm}$ to $40 \mathrm{~mm}$ diameter, $p_{\text {load }}=310^{3} \mathrm{~Pa}$, interstitial-molecular

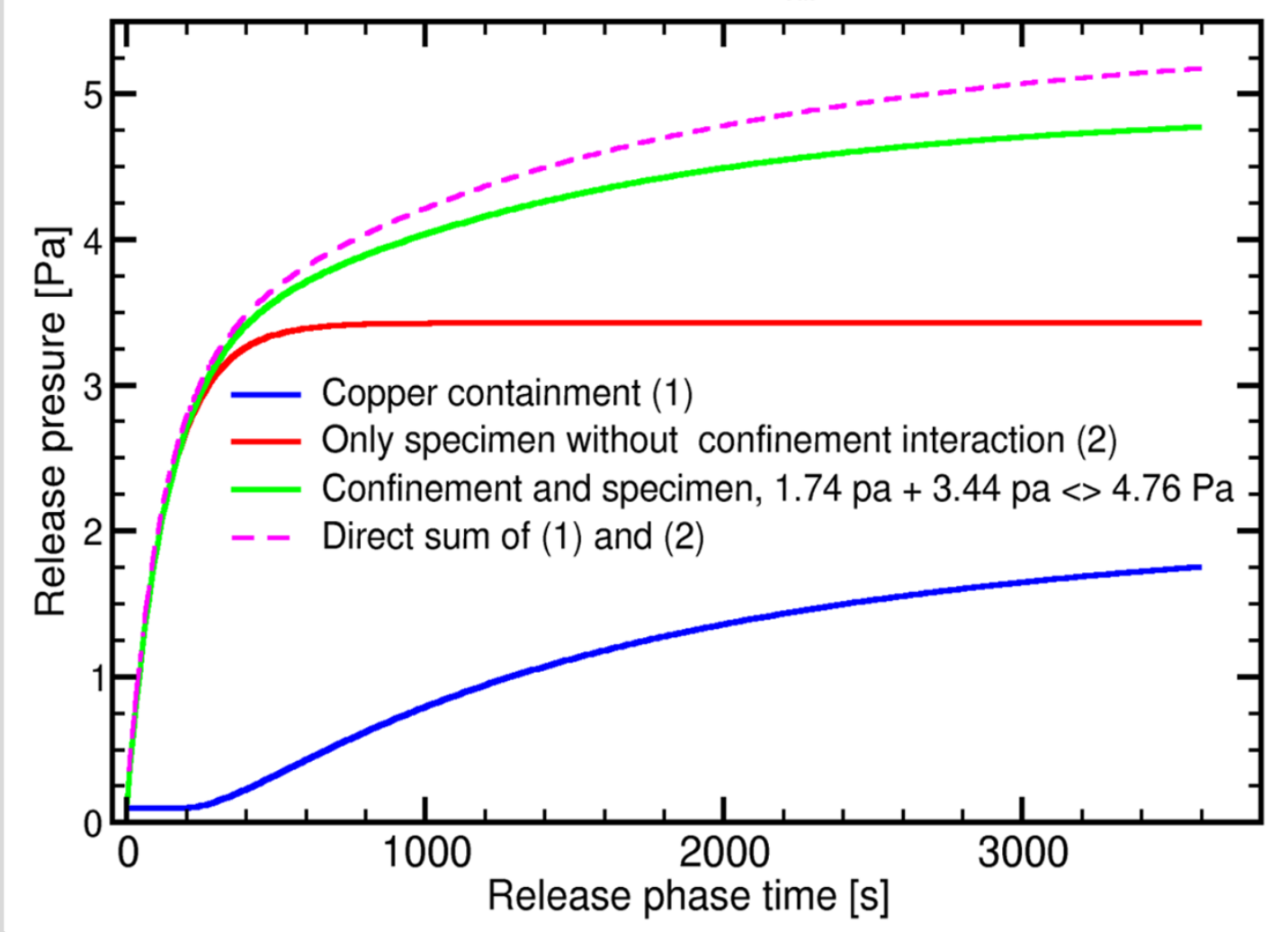

$\mathbb{N}$ CIT

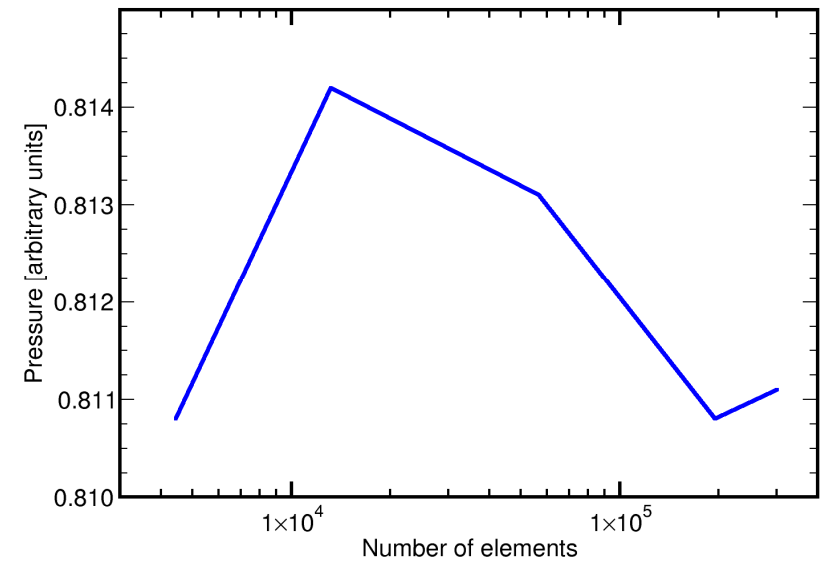

Choosing starting pressure:

Weak pump, half time $10 \mathrm{~s}$, total pressure $10^{5} \mathrm{Pas}, 3 \% \mathrm{D}_{2}$

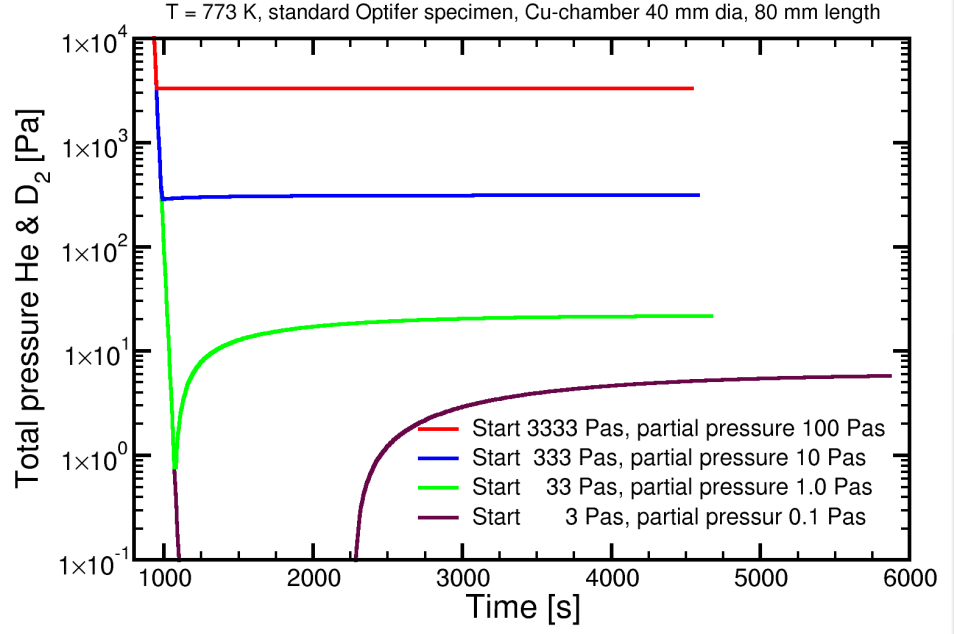




\section{Results for conception of experiments}

$20 \mathrm{~mm}$ diameter, pumping halftime $0.5 \mathrm{~s}, 773 \mathrm{~K}$ Total start pressure $33 \mathrm{Pas}$

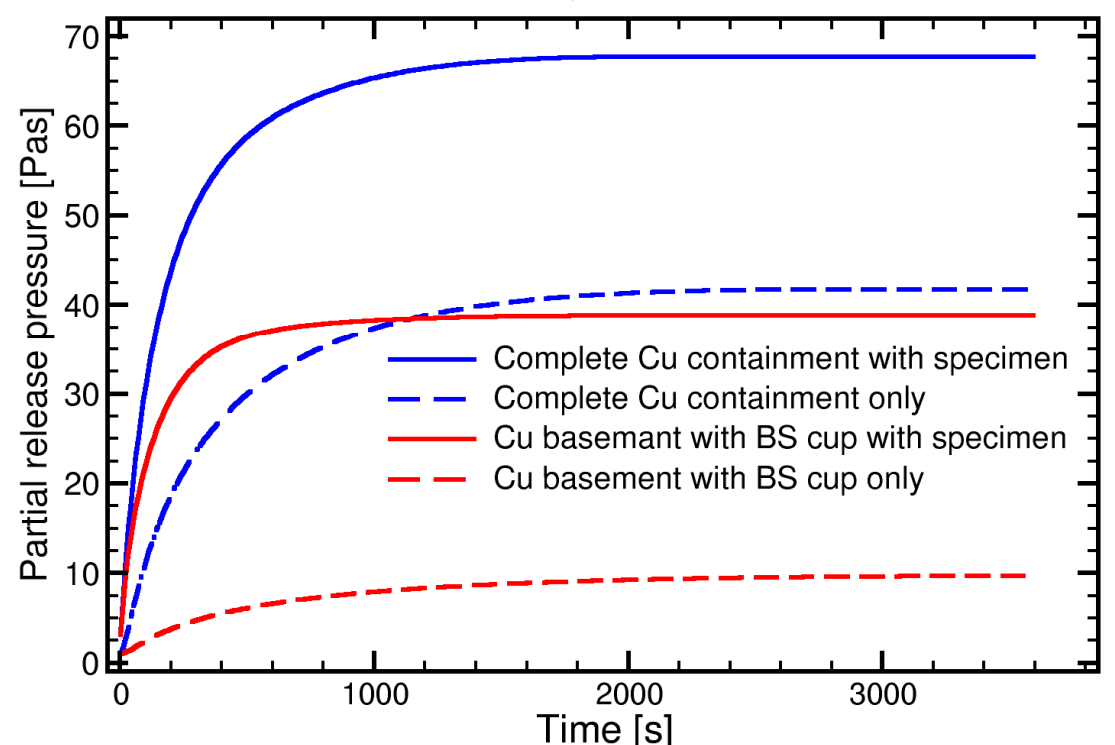

$40 \mathrm{~mm}$ diameter, pumping halftime $0.5 \mathrm{~s}, 773 \mathrm{~K}$

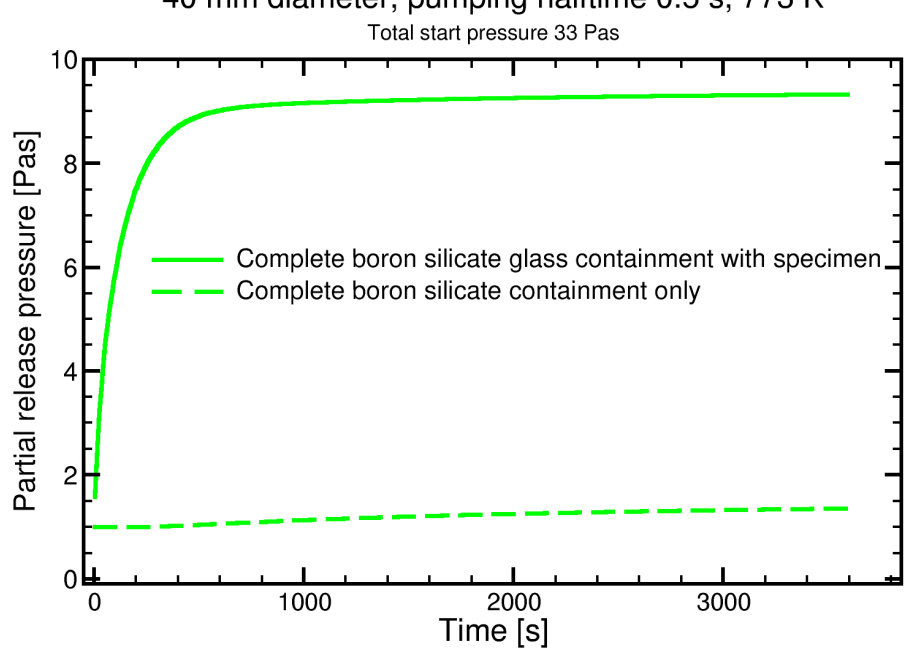

Copper or Boron Silicate glass

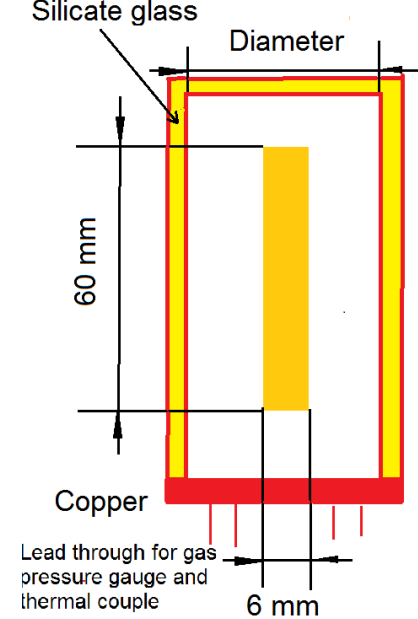

$40 \mathrm{~mm}$ diameter, pumping halftime $0.5 \mathrm{~s}, 773 \mathrm{~K}$ Total start pressure $33 \mathrm{Pas}$

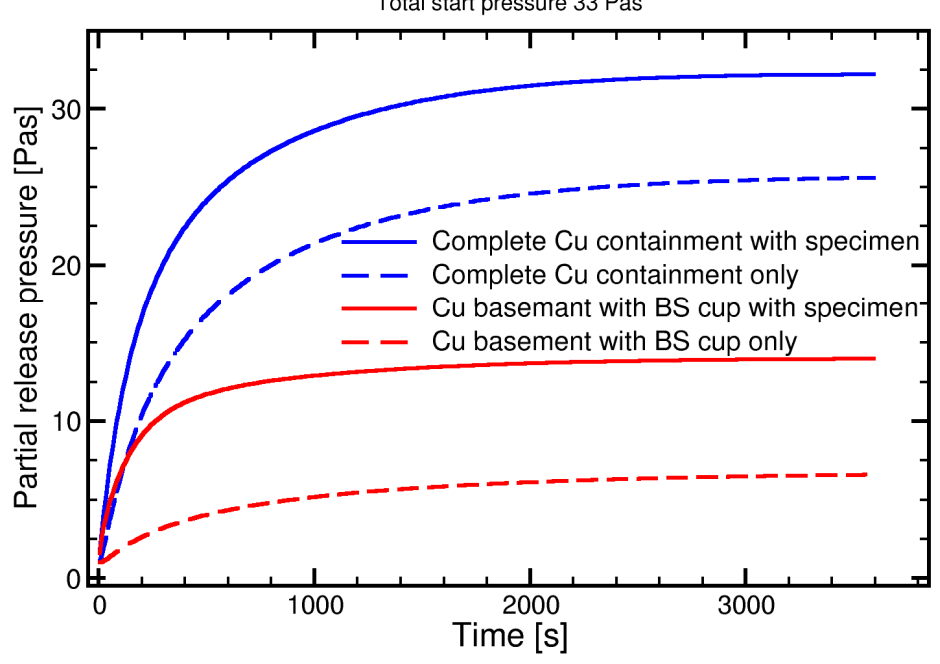

$80 \mathrm{~mm}$ diameter, pumping halftime $0.5 \mathrm{~s}, 773 \mathrm{~K}$

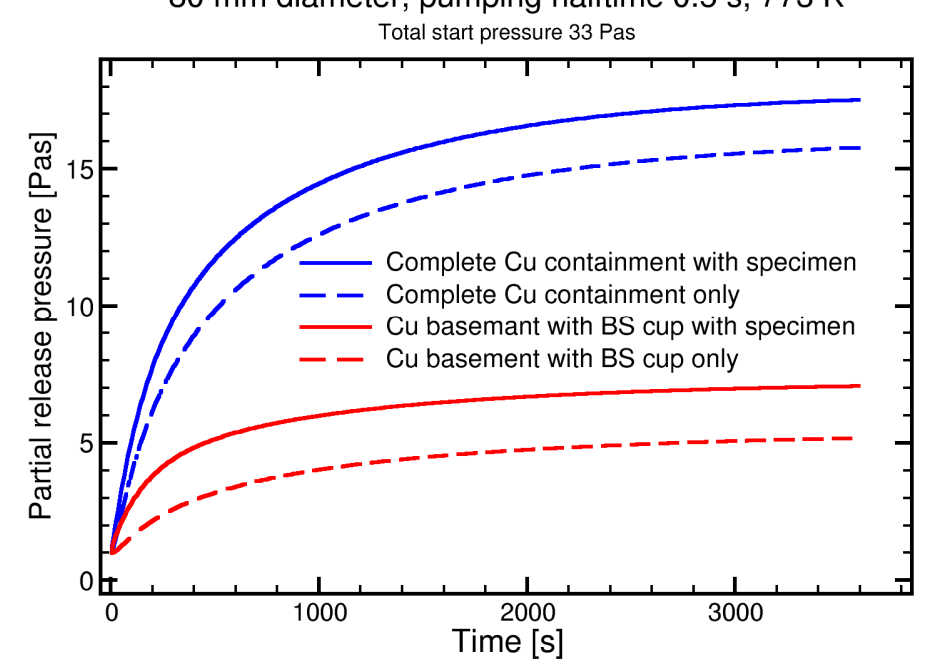




\section{5.: Outlook to analytical solution}

Solution in the charging interval

$c(r, z, t)=k_{s, s a} \sqrt{p_{\text {load }}} \sum_{n, m} \frac{8(-1)^{n+1}}{\pi(2 n+1) x_{m} J_{1}\left(x_{m}\right)} \exp \left(-\gamma_{n, m}^{2} t\right) \cos \left((2 n+1) \frac{\pi}{2} z\right) J_{0}\left(x_{m} r\right)$

where

$\gamma_{n, m}^{2}=D_{s a}\left(\frac{x_{m}^{2}}{r_{s}^{2}}+\frac{(2 n+1)^{2} \pi^{2}}{4 z_{s}^{2}}\right), J_{\alpha}(x)(\alpha=0,1)$ Bessel functions of the first kind,

$x_{m}$ the $m$-th roots of $J_{0}(x)$.
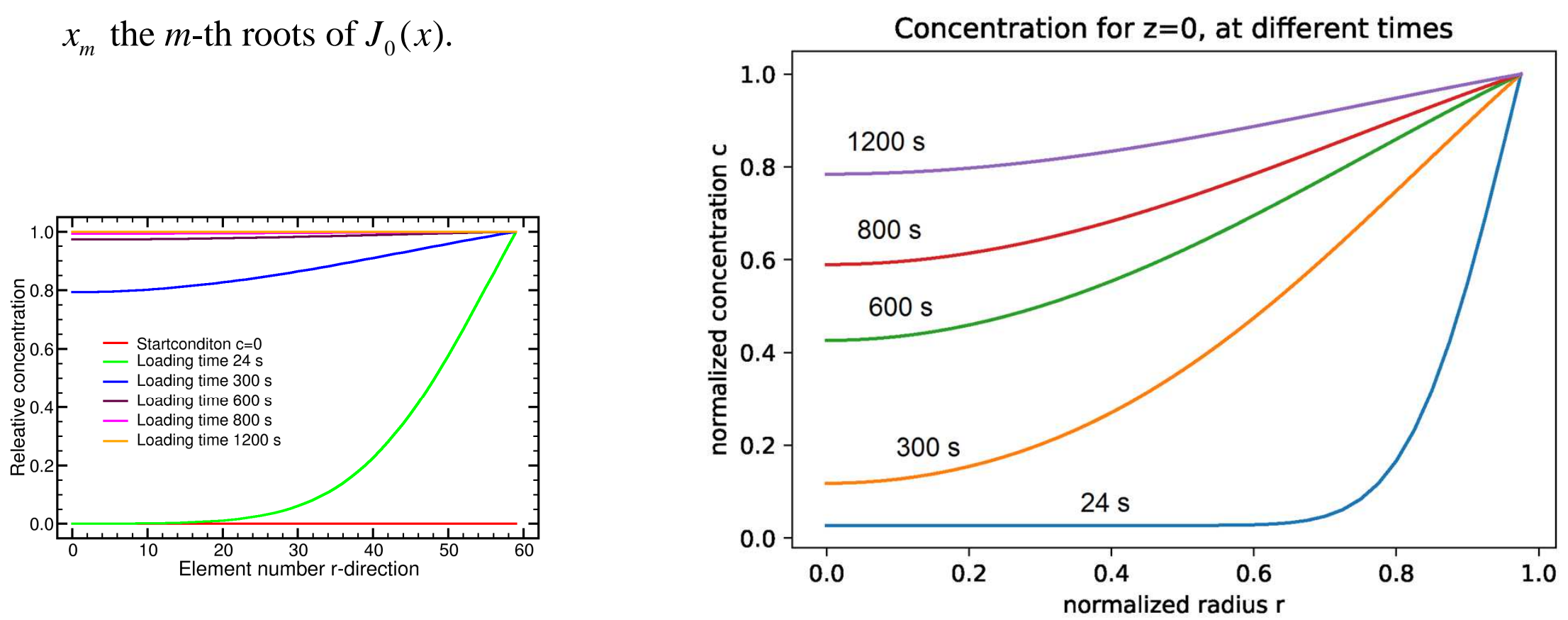